\title{
Electrochemical Synthesis of Dimerizing and Non-Dimerizing Orthoquinone Monoketals
}

Denis Deffieux,${ }^{a, b}$ Isabelle Fabre, ${ }^{a, b}$ Alexander Titz, ${ }^{a, b}$ Jean-Michel Léger ${ }^{c}$ and Stéphane Quideau ${ }^{a, b *}$

${ }^{a}$ Laboratoire de Chimie des Substances Végétales, Centre de Recherche en Chimie Moléculaire, Université Bordeaux 1, 351 cours de la Libération, F-33405 Talence Cedex, ${ }^{b}$ Institut Européen de Chimie et Biologie, Pôle Chimie Organique et Bioorganique, 2 rue Robert Escarpit, F-33607 Pessac Cedex, and ${ }^{c}$ Laboratoire de Chimie Physique et Cristallographie, UFR de Chimie, Université Bordeaux 2, 146 rue Léo Saignat, F-33076 Bordeaux Cedex

s.quideau@iecb.u-bordeaux.fr

\section{Supporting Information}

CONTENTS

- General informations:

S2

- Description for preparation of 1b-d,g; 2b-f; 3b-f; 4d-f,m; 5a-c,i,l; 6a-c,i

with all analytical and spectroscopic data

- ORTEP diagram (CCDC 245748) of the X-ray structure of $\mathbf{6 i}$.

- Detailed PM3 and RHF/3-21G computational data including

HOMO/LUMO energies and atomic coefficients of compounds 4a,c,e,f,

with their Cartesian coordinates and total energies

- Additionnal References

- Proton and carbon NMR spectra of all newly synthesized compounds:

1d ; 2b-f; 3b-f; 4d,f,g,m; 5b,c,i,l; 6a-c,i

$\mathrm{S} 28-\mathrm{S} 82$ 
General. Acetonitrile $(\mathrm{MeCN})$, dichloromethane $\left(\mathrm{CH}_{2} \mathrm{Cl}_{2}\right)$, methanol $(\mathrm{MeOH})$ and tetrahydrofuran (THF) were purified by distillation from $\mathrm{P}_{2} \mathrm{O}_{5}, \mathrm{CaH}_{2}, \mathrm{CaCl}_{2}$ and from sodium/benzophenone, respectively, under $\mathrm{N}_{2}$ immediately before use. Synthesis grade ethyl acetate (EtOAc) was used as received. Light petroleum refers to the fraction boiling in the 40$60^{\circ} \mathrm{C}$ boiling range. Moisture and oxygen sensitive reactions were carried out in flame-dried glassware under $\mathrm{N}_{2}$. Evaporations were conducted under reduced pressure at temperatures less than $45^{\circ} \mathrm{C}$ unless otherwise noted. Column chromatography was carried out under positive pressure using 40-60 $\mu \mathrm{m}$ silica gel and the indicated solvents. Further drying of the residues was accomplished under high vacuum. Melting points are uncorrected. NMR spectra of samples in the indicated solvent were run at 200, 250 or $300 \mathrm{MHz}$. Carbon multiplicities were determined by DEPT 135 experiments.

5-tert-Butyldiphenylsilyloxymethyl-2-methoxyphenol (1b). To a stirring ice-cold suspension of 4-methoxy-3-hydroxybenzyl alcohol (3.01 g, $19.6 \mathrm{mmol})$ in dry $\mathrm{CH}_{2} \mathrm{Cl}_{2}(25$ $\mathrm{mL})$ was added triethylamine $(6 \mathrm{~mL}, 43.1 \mathrm{mmol})$ and 4-dimethymaminopyridine (DMAP, $5.11 \mathrm{~g}, 41.9 \mathrm{mmol})$. The mixture was stirred for $10 \mathrm{~min}$, after which time tertbutyldiphenylsilyl chloride (TBDPSCl, $5.1 \mathrm{~mL}, 19.6 \mathrm{mmol}$ ) was added. After $30 \mathrm{~min}$, the cooling ice bath was removed and the mixture was stirred for $3 \mathrm{~h}$ at room temperature. The solution was diluted in $20 \mathrm{~mL}$ of $\mathrm{CH}_{2} \mathrm{Cl}_{2}$, washed with $1 \mathrm{M} \mathrm{H}_{3} \mathrm{PO}_{4}(3 \times 10 \mathrm{~mL})$ and brine $(3 \times$ $10 \mathrm{~mL}$ ). The organic layer was dried over $\mathrm{Na}_{2} \mathrm{SO}_{4}$, filtered and evaporated to give crude $\mathbf{1 b}$ as a yellow oil, $(6.14 \mathrm{~g}, 80 \%)$. All spectrometric data were identical to those previously reported. $^{6 a}$

5-Bromoguaiacol (1c). A 39\% solution of peracetic acid in acetic acid (30 mL, $446 \mathrm{mmol})$ was added dropwise to a stirring ice-cooled solution of 5-bromo-2-methoxybenzaldehyde $(15.0 \mathrm{~g}, 70.0 \mathrm{mmol})$ in EtOAc $(160 \mathrm{~mL})$. The reaction mixture was allowed to warm up slowly to room temperature while stirred overnight, after which time it was diluted in $\mathrm{H}_{2} \mathrm{O}(80$ $\mathrm{mL})$. After separation, the aqueous layer was extracted with EtOAc $(3 \times 20 \mathrm{~mL})$, and the combined organic layers were washed with saturated aqueous $\mathrm{NaHCO}_{3}(3 \times 10 \mathrm{~mL})$, dried 
over $\mathrm{Na}_{2} \mathrm{SO}_{4}$, filtered and evaporated. The yellow residue was dissolved in $\mathrm{MeOH}(160 \mathrm{~mL})$, then $\mathrm{KOH}$ pellets $(4.12 \mathrm{~g}, 73.6 \mathrm{mmol})$ were added portionwise at $0^{\circ} \mathrm{C}$ under stirring. The reaction mixture was stirred at $0^{\circ} \mathrm{C}$ for an additional $30 \mathrm{~min}$, after which time it was diluted in $\mathrm{H}_{2} \mathrm{O}(80 \mathrm{~mL})$, slowly acidified with $10 \%$ aqueous $\mathrm{HCl}$, extracted with EtOAc $(3 \times 20 \mathrm{~mL})$, washed with brine $(2 \times 10 \mathrm{~mL})$, dried over $\mathrm{Na}_{2} \mathrm{SO}_{4}$, filtered and evaporated to give crude $1 \mathbf{c}$ as a yellow oil, which was purified by column chromatography, eluting with light petroleum $/ \mathrm{Et}_{2} \mathrm{O}(6: 1 \rightarrow 3: 1)$ to furnish $1 \mathrm{c}(9.2 \mathrm{~g}, 65 \%)$ as an off-white solid. All characterization data were identical to those previously reported. ${ }^{22}$

4-tert-Butyldiphenylsilyloxymethyl-2-methoxyphenol (1d). To a stirring ice-cold suspension of 3-methoxy-4-hydroxybenzyl alcohol (3 g, $19.5 \mathrm{mmol})$ in dry $\mathrm{CH}_{2} \mathrm{Cl}_{2}(20 \mathrm{~mL})$ was added triethylamine $(6 \mathrm{~mL}, 43.13 \mathrm{mmol})$ and DMAP ( $5 \mathrm{~g}, 41.6 \mathrm{mmol})$. The mixture was stirred for $10 \mathrm{~min}$, after which time TBDPSCl (3.2 mL, $12.3 \mathrm{mmol})$ was added. After $30 \mathrm{~min}$, the cooling ice bath was removed and the mixture was stirred for $3 \mathrm{~h}$ at room temperature. The solution was diluted in $20 \mathrm{~mL}$ of $\mathrm{CH}_{2} \mathrm{Cl}_{2}$, washed with $1 \mathrm{M} \mathrm{H}_{3} \mathrm{PO}_{4}(3 \times 10 \mathrm{~mL})$ and brine $(3 \times 10 \mathrm{~mL})$. The organic layer was dried over $\mathrm{Na}_{2} \mathrm{SO}_{4}$, filtered and evaporated to give crude 1d as a yellow oil, which was purified by column chromatography, eluting with light petroleum/ $\mathrm{Et}_{2} \mathrm{O}(1: 1)$ to furnish pure $1 \mathrm{~d}$ as a yellow oil $(4.23 \mathrm{~g}, 54 \%)$ : IR $(\mathrm{NaCl}) 3535 \mathrm{~cm}^{-1}$; ${ }^{1} \mathrm{H}$ NMR $\left(\mathrm{CDCl}_{3}, 250 \mathrm{MHz}\right) \delta 1.11(\mathrm{~s}, 9 \mathrm{H}), 3.86(\mathrm{~s}, 3 \mathrm{H}), 4.72(\mathrm{~s}, 2 \mathrm{H}), 5.56(\mathrm{~s}, 1 \mathrm{H}), 6.78-6.90$ $(\mathrm{m}, 3 \mathrm{H}), 7.35-7.73(\mathrm{~m}, 10 \mathrm{H}) ;{ }^{13} \mathrm{C} \mathrm{NMR}\left(\mathrm{CDCl}_{3}, 62.9 \mathrm{MHz}\right) \delta 146.3,144.5,135.6,134.7$, 133.6, 129.6, 127.7, 119.0, 114.0, 109.0, 65.5, 55.8, 26.8, 19.3; LSIMS $m / z$ (rel intensity) 415 ( $\left.\mathrm{MNa}^{+}, 9\right), 391$ (19), 335 (100); HRMS (LSIMS) calcd for $\mathrm{C}_{24} \mathrm{H}_{28} \mathrm{O}_{3} \mathrm{SiNa} 415.1705$ found 415.1708 .

\section{4-tert-Butyldiphenylsilyloxymethyl-6,6-dimethoxycyclohexa-2,4-dienone}

(4d).

Electrochemical oxidation of 4-tert-butyldiphenylsilyloxymethyl-2-methoxyphenol 1d (466 $\mathrm{mg}, 1.19 \mathrm{mmol}$ ) was performed at $100 \mathrm{~mA}$ according to the general procedure A. The residue was purified by column chromatography, eluting with light petroleum/ $\mathrm{Et}_{2} \mathrm{O}(1: 1)$, to furnish pure orthoquinone monoketal $\mathbf{4 d}$ as a yellow oil (502 mg, 100\%); IR ( $\mathrm{NaCl}) 1682,2314$, 
2872, $2957 \mathrm{~cm}^{-1} ;{ }^{1} \mathrm{H} \mathrm{NMR}\left(\mathrm{CDCl}_{3}, 250 \mathrm{MHz}\right) \delta 1.10(\mathrm{~s}, 9 \mathrm{H}), 3.36(\mathrm{~d}, J=3.7 \mathrm{~Hz}, 6 \mathrm{H}), 4.32(\mathrm{~s}$, 2H), $6.01(\mathrm{dd}, J=2.7,10.1 \mathrm{~Hz}, 1 \mathrm{H}), 6.41(\mathrm{~d}, J=1.5 \mathrm{~Hz}, 1 \mathrm{H}), 6.71(\mathrm{~d}, J=9.8 \mathrm{~Hz}, 1 \mathrm{H}), 7.40-$ $7.72(\mathrm{~m}, 10 \mathrm{H}) ;{ }^{13} \mathrm{C} \mathrm{NMR}\left(\mathrm{CDCl}_{3}, 62.9 \mathrm{MHz}\right) \delta ; 195.1,139.1,136.9,135.3,132.6,129.8$, 128.2, 127.7, 125.7, 91.5, 64.3, 49.8, 26.6, 19.0; EIMS $m / z$ (rel intensity) $422\left(\mathrm{M}^{+}, 29\right), 365$ (7), 213 (29), 183 (11), 167 (10); HRMS (EIMS) calcd for $\mathrm{C}_{25} \mathrm{H}_{30} \mathrm{O}_{4} \mathrm{Si} 422.1913$, found 422.1914 .

4-Bromo-6,6-dimethoxycyclohexa-2,4-dienone (4e). Electrochemical oxidation of 4-bromo2-methoxyphenol $1 \mathbf{e}^{23}(171 \mathrm{mg}, 0.84 \mathrm{mmol})$ was performed at $50 \mathrm{~mA}$ according to the procedure A. The residue was purified by column chromatography, eluting with light petroleum/ $\mathrm{Et}_{2} \mathrm{O}(2: 1)$, to furnish pure orthoquinone monoketal $4 \mathbf{e}$ as a yellow oil (126 mg, $64 \%$ ). All spectroscopic data were identical to those previously reported. ${ }^{8 b, 12 b}$

5,6,6-Trimethoxycyclohexa-2,4-dienone (4f). Electrochemical oxidation of 2,3dimethoxyphenol 1f (190 mg, $1.23 \mathrm{mmol}$ ) was performed at $100 \mathrm{~mA}$ according to the procedure A. The residue was purified by column chromatography, eluting with pure $\mathrm{Et}_{2} \mathrm{O}$, to furnish pure orthoquinone monoketal $4 \mathbf{f}^{1 \mathrm{a}, \mathrm{c}}$ as a yellow oil (226 mg, 100\%); IR ( NaCl) 1560, 1642, $1682 \mathrm{~cm}^{-1} ;{ }^{1} \mathrm{H}$ NMR $\left(\mathrm{CDCl}_{3}, 300 \mathrm{MHz}\right) \delta 3.24$ (s, 6H), 3.73 (s, 3H), $5.35(\mathrm{bd}, 1 \mathrm{H})$, 5.77 (bt, 1H), 6.95 (bt, 1H); ${ }^{13} \mathrm{C} \mathrm{NMR}\left(\mathrm{CDCl}_{3}, 62.9 \mathrm{MHz}\right) \delta 194.5,163.3,142.9,118.9,96.3$, 93.8, 56.0, 51.3; EIMS m/z (rel intensity) $184\left(\mathrm{M}^{+}, 70\right), 169$ (41), 153 (22); HRMS (EIMS) calcd for $\mathrm{C}_{9} \mathrm{H}_{12} \mathrm{O}_{4}$ 184.0736, found 184.0735.

\section{3,3,10,10-Tetramethoxytricyclo $\left[6.2 .2 .0^{2,7}\right]$ dodeca-5,11-diene-4,9-dione}

(5a).

Electrochemical oxidation of guaiacol 1a $(211 \mathrm{mg}, 1.70 \mathrm{mmol})$ was performed at $100 \mathrm{~mA}$ according to the procedure A. The residue was purified by column chromatography, eluting with light petroleum/ $\mathrm{Et}_{2} \mathrm{O}[(2: 1) \rightarrow(1: 1)]$, to furnish pure dimer 5a as fine off-white crystals (241 mg, 92\%). All spectroscopic data were identical to those previously reported. ${ }^{8 \mathrm{a}, \mathrm{b}, 10 \mathrm{f}}$ 


\section{6,12-bis-(tert-Butyldiphenylsilyloxymethyl)-3,3,10,10-}

tetramethoxytricyclo[6.2.2.0 $\left.{ }^{2.7}\right]$ dodeca-5,11-diene-4,9-dione $\quad(5 b)$ Electrochemical oxidation of 5-tert-butyldiphenylsilyloxymethyl-2-methoxyphenol $\mathbf{1 b}$ (494 mg, $1.26 \mathrm{mmol}$ ) was performed at $100 \mathrm{~mA}$ according to the procedure A. The residue was purified by column chromatography, eluting with light petroleum/ $\mathrm{Et}_{2} \mathrm{O}(1: 1)$, to furnish pure dimer $\mathbf{5 b}$ as a brown oil (414 mg, 78\%); IR (NaCl) 1742, $1707 \mathrm{~cm}^{-1} ;{ }^{1} \mathrm{H}$ NMR $\left(\mathrm{CDCl}_{3}, 300 \mathrm{MHz}\right) \delta 1.04(\mathrm{~s}, 9 \mathrm{H})$, $1.06(\mathrm{~s}, 9 \mathrm{H}), 3.11(\mathrm{~s}, 3 \mathrm{H}), 3.19$ (d, $J=6.6 \mathrm{~Hz}, 1 \mathrm{H}), 3.28(\mathrm{~s}, 2 \mathrm{H}), 3.30(\mathrm{~s}, 3 \mathrm{H}), 3.46(\mathrm{~s}, 6 \mathrm{H})$, $3.91(\mathrm{~s}, 2 \mathrm{H}), 4.21(\mathrm{q}, J=16.7 \mathrm{~Hz}, 2 \mathrm{H}), 6.21(\mathrm{~d}, J=6.6 \mathrm{~Hz}, 1 \mathrm{H}), 6.32(\mathrm{~s}, 1 \mathrm{H}), 7.28-7.65$ (m, 20 $\mathrm{H}) ;{ }^{13} \mathrm{C}$ NMR $\left(\mathrm{CDCl}_{3}, 75.5 \mathrm{MHz}\right) 201.3,193.2$, 157.7, 139.6, 135.4, 135.3, 135.2, 135.1, 133.0, 132.6, 132.4, 129.9, 129.7, 127.7, 124.4, 123.2, 98.8, 94.7, 64.5, 64.0, 53.8, 50.4, 49.9, 49.5, 48.9, 39.4, 38.7, 26.6, 26.5, 19.1, 19.0; LSIMS $m / z$ (rel intensity) 867 ( $\left.\mathrm{MNa}^{+}, 100\right), 199$ (41); HRMS (LSIMS) calcd for $\mathrm{C}_{50} \mathrm{H}_{60} \mathrm{O}_{8} \mathrm{SiNa}$ 867.3724, found 867.3719.

\section{6,12-Dibromo-3,3,10,10-tetramethoxytricyclo[6.2.2.0 $\left.{ }^{2,7}\right]$ dodeca-5,11-diene-4,9-dione (5c).} Electrochemical oxidation of 5-bromoguaiacol 4e (226 mg, $1.12 \mathrm{mmol})$ was performed at 50 $\mathrm{mA}$ according to the procedure A. The residue was purified by column chromatography, eluting with light petroleum/ $\mathrm{Et}_{2} \mathrm{O}(1: 1)$, to furnish pure dimer $\mathbf{5 c}$ as fine beige crystals $(260$ $\mathrm{mg}, 100 \%), \mathrm{mp} 154^{\circ} \mathrm{C}$; IR (NaCl) 1438, 1615, 1716, $1750 \mathrm{~cm}^{-1} ;{ }^{1} \mathrm{H} \mathrm{NMR}\left(\mathrm{CDCl}_{3}, 250 \mathrm{MHz}\right)$ $\delta 3.10-3.22(\mathrm{~m}, 2 \mathrm{H}), 3.10(\mathrm{~s}, 3 \mathrm{H}), 3.22(\mathrm{~s}, 3 \mathrm{H}), 3.37(\mathrm{~s}, 3 \mathrm{H}), 3.45(\mathrm{~s}, 3 \mathrm{H}), 3.65(\mathrm{~d}, J=1.8 \mathrm{~Hz}$, 1H), $3.86(\mathrm{~s}, 1 \mathrm{H}), 6.34(\mathrm{~d}, J=1.8 \mathrm{~Hz}, 1 \mathrm{H}), 6.56(\mathrm{~s}, 1 \mathrm{H}) ;{ }^{13} \mathrm{C} \mathrm{NMR}\left(\mathrm{CDCl}_{3}, 62.9 \mathrm{MHz}\right) \delta$ $198.9,190.1,144.5,132.7,130.7,118.0,98.2$, 94.3, 61.7, 50.8, 50.5, 49.8, 49.0, 48.6, 42.2, 39.3; EIMS m/z (rel intensity) 439 (12), 438 (73), 437 (8), 327 (12), 325 (12), 299 (97), 297 (100). HRMS (LSIMS) calcd for $\mathrm{C}_{16} \mathrm{H}_{18} \mathrm{O}_{6} \mathrm{Br}_{2} \mathrm{Na} 486.9368$ found 486.9380 .

2,6-Dimethoxy-1,4-benzoquinone. Electrochemical oxidation of 2,6-dimethoxyphenol $\mathbf{1 g}$ (283 $\mathrm{mg}, 1.84 \mathrm{mmol}$ ) was performed at $100 \mathrm{~mA}$ according to the procedure A. The residue was purified by column chromatography, eluting with pure $\mathrm{CH}_{2} \mathrm{Cl}_{2}$, to furnish 2,6-dimethoxy1,4-benzoquinone as a yellow solid (94 mg, 30\%), mp 254-256 ${ }^{\circ} \mathrm{C}\left(\right.$ lit. $\left.^{24} \mathrm{mp} 252-254^{\circ} \mathrm{C}\right)$; IR $(\mathrm{NaCl}) 1600,1645,1695,1321 \mathrm{~cm}^{-1} ;{ }^{1} \mathrm{H} \mathrm{NMR}\left(\mathrm{CDCl}_{3}, 250 \mathrm{MHz}\right) \delta 3.81(\mathrm{~s}, 6 \mathrm{H}), 5.85(\mathrm{~s}$, 
$2 \mathrm{H}) ;{ }^{13} \mathrm{C} \mathrm{NMR}\left(\mathrm{CDCl}_{3}, 62.9 \mathrm{MHz}\right) \delta 186.6,157.3,107.4,56.5$; EIMS $m / z$ (rel intensity) 168 $\left(\mathrm{M}^{+}, 38\right), 137$ (13), 69 (100).

2-(4-propyl-2-methoxyphenoxy)-2-methylpropionic acid (2b). Hydrogenation of eugenol (20 g, $0.122 \mathrm{~mol})$ in dry THF $(150 \mathrm{~mL})$ was carried out under $\mathrm{H}_{2}$ (balloon) for $24 \mathrm{~h}$ at room temperature in the presence of $10 \mathrm{wt} \% \mathrm{Pd} / \mathrm{C}$ as a catalyst $(1.5 \mathrm{~g})$. The reaction mixture was filtered through Celite, and the solid was washed with acetone. Evaporation of the combined filtrates and washings afforded an oil (20 g), which was used without further purification. To a stirring ice-cold solution of this crude 2-methoxy-4-propylphenol (5.3 g, $31.5 \mathrm{mmol})$ and 1,1,1-trichloro-2-methylpropan-2-ol $\left(\mathrm{H}_{2} \mathrm{O}\right)_{\mathrm{x}}(16.7 \mathrm{~g}, 94.1 \mathrm{mmol})^{8 \mathrm{a}, 25}$ in acetone $(120 \mathrm{~mL})$ was added powdered sodium hydroxide $(6.6 \mathrm{~g}, 165 \mathrm{mmol})$ in three equal portions at $2 \mathrm{~h}$ intervals. After each addition, the reaction mixture was allowed to warm to room temperature. Before the last addition, an additional $40 \mathrm{~mL}$ of acetone added to the thick suspension. The reaction mixture was then refluxed for $64 \mathrm{~h}$. The solvent was evaporated to give a residue, which was diluted in water and acidified to $\mathrm{pH} 1$ with $10 \%$ aq $\mathrm{HCl}$. The aqueous phase was extracted with $\mathrm{CH}_{2} \mathrm{Cl}_{2}(3 \times 50 \mathrm{~mL})$. The organic layers were then dried over $\mathrm{Na}_{2} \mathrm{SO}_{4}$, filtered, and evaporated to give crude $\mathbf{2 b}$, which was submitted to column chromatography, eluting with light petroleum/EtOAc $(8: 1 \rightarrow 2: 1)$, to furnish pure $\mathbf{2 b}$ as a yellow oil $(1.97 \mathrm{~g}, 25 \%)$; IR $(\mathrm{NaCl})$ 3314, 1767, $1713 \mathrm{~cm}^{-1} ;{ }^{1} \mathrm{H}$ NMR $\left(\mathrm{CDCl}_{3}, 250 \mathrm{MHz}\right) \delta 0.92(\mathrm{t}, J=7.32 \mathrm{~Hz}, 3 \mathrm{H}), 1.49(\mathrm{~s}, 6 \mathrm{H})$, $1.61(\mathrm{q}, J=7.63,7.33 \mathrm{~Hz}, 2 \mathrm{H}), 2.53(\mathrm{t}, J=7.63 \mathrm{~Hz}, 2 \mathrm{H}), 3.84(\mathrm{~s}, 3 \mathrm{H}), 6.67-6.91(\mathrm{~m}, 3 \mathrm{H}) ;{ }^{13} \mathrm{C}$ $\mathrm{NMR}\left(\mathrm{CDCl}_{3}, 62.9 \mathrm{MHz}\right) \delta 176.5,152.0,140.4,140.1,123.2,120.6,111.9,81.9,55.5,37.7$, 24.7, 24.4, 13.6; LSIMS $m / z$ (rel intensity) $275\left(\mathrm{MNa}^{+}, 100\right), 253\left(\mathrm{M}^{+}, 58\right)$; HRMS (LSIMS) calcd for $\mathrm{C}_{14} \mathrm{H}_{20} \mathrm{O}_{4} \mathrm{Na} 275.1259$ found 275.1257.

\section{2-(4-tert-Butyldiphenylsilyloxymethyl-2-methoxyphenoxy)-2-methylpropionic acid (2c).}

A stirred ice-cold solution of $\mathbf{1 d}(2.10 \mathrm{~g}, 5.40 \mathrm{mmol})$ and 1,1,1-trichloro-2-methylpropan-2-ol $\left(\mathrm{H}_{2} \mathrm{O}\right)_{\mathrm{x}}(2.90 \mathrm{~g}, 16.3 \mathrm{mmol})^{8 \mathrm{a}, 25}$ in acetone $(50 \mathrm{~mL})$ was treated with powdered sodium hydroxide (1.3 g, $32.5 \mathrm{mmol})$ and processed as described for $\mathbf{2 b}$. The resulting crude $\mathbf{2} \mathbf{c}$ was submitted to column chromatography, eluting with hexanes/EtOAc $(6: 1 \rightarrow 2: 1)$, to furnish $2 \mathbf{c}$ 
as a transparent oil, which was crystallized from $\mathrm{CH}_{2} \mathrm{Cl}_{2}$ to give $\mathbf{2 c}$ as fine white crystals (1.93 g, 54\%), mp 77-78 ${ }^{\circ}$; IR ( $\left.\mathrm{NaCl}\right) 3330,1765,1719,1590 \mathrm{~cm}^{-1} ;{ }^{1} \mathrm{H}$ NMR $\left(\mathrm{CDCl}_{3}, 250 \mathrm{MHz}\right) \delta$ $1.11(\mathrm{~s}, 9 \mathrm{H}), 1.52(\mathrm{~s}, 6 \mathrm{H}), 3.85(\mathrm{~s}, 3 \mathrm{H}), 4.75(\mathrm{~s}, 2 \mathrm{H}), 6.82-6.98(\mathrm{~m}, 3 \mathrm{H}), 7.35-7.70(\mathrm{~m}, 10 \mathrm{H})$; ${ }^{13} \mathrm{C}$ NMR $\left(\mathrm{CDCl}_{3}, 62.9 \mathrm{MHz}\right) \delta 175.7,152.3,141.1,138.8,135.6,133.3,129.8,127.7,123.5$, 118.3, 109.6, 82.6, 65.1, 55.6, 26.8, 24.9, 19.3; LSIMS $\mathrm{m} / z$ (rel intensity) $501\left(\mathrm{MNa}^{+}, 63\right)$, 433 (42); HRMS (LSIMS) calcd for $\mathrm{C}_{28} \mathrm{H}_{34} \mathrm{SiO}_{5} \mathrm{Na} 501.2073$, found 501.2070.

\section{2-(5-tert-Butyldiphenylsilyloxymethyl-2-methoxyphenoxy)-2-methylpropionic acid (2d).}

A stirred ice-cold solution of $\mathbf{1 b}(2.87 \mathrm{~g}, 7.3 \mathrm{mmol})$ and 1,1,1-trichloro-2-methylpropan-2-ol $\left(\mathrm{H}_{2} \mathrm{O}\right)_{\mathrm{x}}(3.97 \mathrm{~g}, 22.4 \mathrm{mmol})^{8 \mathrm{a}, 25}$ in acetone $(50 \mathrm{~mL})$ was treated with powdered sodium hydroxide (1.3 g, $32.5 \mathrm{mmol})$ and processed as described for $\mathbf{2 b}$. The resulting crude $\mathbf{2 d}$ was submitted to column chromatography, eluting with hexanes/EtOAc $(6: 1 \rightarrow 2: 1)$, to furnish pure 2d as a transparent oil (1.39 g, 40\%); IR (NaCl) 3328, 1765, 1715, $1265 \mathrm{~cm}^{-1}$; ${ }^{1} \mathrm{H}$ NMR $\left(\mathrm{CDCl}_{3}, 250 \mathrm{MHz}\right) \delta 1.14(\mathrm{~s}, 9 \mathrm{H}), 1.55(\mathrm{~s}, 6 \mathrm{H}), 3.88(\mathrm{~s}, 3 \mathrm{H}), 4.74(\mathrm{~s}, 2 \mathrm{H}), 6.88-7.09(\mathrm{~m}, 3 \mathrm{H})$, 7.71-7.74 (m, 10H), $9.75(\mathrm{bs}, 1 \mathrm{H}) ;{ }^{13} \mathrm{C} \mathrm{NMR}\left(\mathrm{CDCl}_{3}, 62.9 \mathrm{MHz}\right) \delta 176.7,151.2,142.5,135.4$, $134.0,133.3,129.7,129.6,127.7,127.6,122.6,121.5,111.5,81.9,79.8,64.7,55.7,26.7$, 24.8, 19.2; EIMS m/z (rel intensity) $478\left(\mathrm{M}^{+}, 2\right), 421$ (39), 335 (58), 199 (38), 137 (100); HRMS (EIMS) calcd for $\mathrm{C}_{28} \mathrm{H}_{34} \mathrm{SiO}_{5} 478.2175$, found 478.2175 .

2-(4-bromo-2-methoxyphenoxy)-2-methylpropionic acid (2e). A stirred ice-cold solution of 1e $(1.20 \mathrm{~g}, 5.91 \mathrm{mmol})$ and 1,1,1-trichloro-2-methylpropan-2-ol $\left(\mathrm{H}_{2} \mathrm{O}\right)_{\mathrm{x}}(3.18 \mathrm{~g}, 17.9$ $\mathrm{mmol})^{8 \mathrm{a}, 25}$ in acetone $(50 \mathrm{~mL})$ was treated with powdered sodium hydroxide $(1.45 \mathrm{~g}, 36.2$ mmol) and processed as described for $\mathbf{2} \mathbf{b}$. The resulting crude $\mathbf{7 e}$ was submitted to column chromatography, eluting with light petroleum/ $\mathrm{Et}_{2} 0(2: 1)$, to furnish pure $7 \mathbf{e}$ as a transparent oil (753 mg, 44\%); IR (NaCl) 3348, 1763, $1718 \mathrm{~cm}^{-1} ;{ }^{1} \mathrm{H}$ NMR $\left(\mathrm{CDCl}_{3}, 250 \mathrm{MHz}\right) \delta 1.50(\mathrm{~s}$, $6 \mathrm{H}), 3.80(\mathrm{~s}, 3 \mathrm{H}), 6.74-7.00(\mathrm{~m}, 3 \mathrm{H}), 10.7(\mathrm{bs}, 1 \mathrm{H}) ;{ }^{13} \mathrm{C} \mathrm{NMR}\left(\mathrm{CDCl}_{3}, 62.9 \mathrm{MHz}\right) \delta 177.5$, 153.0, 142.3, 124.2, 123.6, 117.0, 115.4, 81.5, 55.8, 24.6; LSIMS $m / z$ (rel intensity) 313 $\left(\mathrm{MNa}^{+}, 22\right), 311\left(\mathrm{MNa}^{+}, 22\right), 290\left(\mathrm{MH}^{+}, 37\right), 288\left(\mathrm{MH}^{+}, 34\right), 245$ (98), 243 (100); HRMS (LSIMS) calcd for $\mathrm{C}_{11} \mathrm{H}_{13} \mathrm{BrO}_{4} 287.9997$, found 287.9999 . 
2-(5-bromo-2-methoxyphenoxy)-2-methylpropionic acid (2f). A stirred ice-cold solution of $1 c(1.1 \mathrm{~g}, 5.50 \mathrm{mmol})$ and 1,1,1-trichloro-2-methylpropan-2-ol $\left(\mathrm{H}_{2} \mathrm{O}\right)_{\mathrm{x}}(2.9 \mathrm{~g}, 16.50 \mathrm{mmol})^{8 \mathrm{a}, 25}$ in acetone $(40 \mathrm{~mL})$ was treated with powdered sodium hydroxide $(1.4 \mathrm{~g}, 34.37 \mathrm{mmol})$ and processed as described for $\mathbf{2 b}$. The resulting crude $\mathbf{2 f}$ was submitted to column chromatography, eluting with light petroleum/ $\operatorname{Et}_{2} 0(2: 1)$, to furnish pure $\mathbf{2 f}$ as a transparent oil (598 mg, 38\%); IR (NaCl) 3348, 1763, $1718 \mathrm{~cm}^{-1} ;{ }^{1} \mathrm{H}$ NMR $\left(\mathrm{CDCl}_{3}, 250 \mathrm{MHz}\right) \delta 1.50(\mathrm{~s}$, $6 \mathrm{H}), 3.78(\mathrm{~s}, 3 \mathrm{H}), 6.73-7.16(\mathrm{~m}, 1 \mathrm{H}), 10.24(\mathrm{bs}, 1 \mathrm{H}) ;{ }^{13} \mathrm{C} \mathrm{NMR}\left(\mathrm{CDCl}_{3}, 62.9 \mathrm{MHz}\right) \delta 177.4$, 151.7, 143.9, 127.4, 126.0, 113.2, 112.1, 81.7, 55.7, 24.6; LSIMS $m / z$ (rel intensity) 311 $\left(\mathrm{MNa}^{+}, 22\right), 290\left(\mathrm{MH}^{+}, 37\right), 243$ (100), 202 (71); HRMS (LSIMS) calcd for $\mathrm{C}_{11} \mathrm{H}_{13} \mathrm{BrO}_{4}$ 287.9997, found 287.9999.

\section{3,3-dimethyl-1,4-dioxaspiro[4.5]deca-7,9-diene-6,6-dimethoxy-8-propyl-2-one}

(3b).

Oxidation of the carboxylic acid $\mathbf{2 b}(740 \mathrm{mg}, 2.9 \mathrm{mmol})$ was performed in $\mathrm{CH}_{3} \mathrm{CN} / \mathrm{MeOH}$ $(9: 1)$ in the presence of pyridine ( 2 equiv) according to the general procedure $\mathrm{A}$ at a constant current of $30 \mathrm{~mA}$ with passage of $2.4 \mathrm{~F} / \mathrm{mol}$. The residue was further dried overnight to give an oily crude product, which was purified by column chromatography, eluting hexane/EtOAc (4:1), to furnish the spirolactone bisketal $\mathbf{3 b}$ as a pale yellow oil (413 $\mathrm{mg}, 50 \%)$; IR $(\mathrm{NaCl})$ 2969, 2935, $1794 \mathrm{~cm}^{-1} ;{ }^{1} \mathrm{H}$ NMR $\left(\mathrm{CDCl}_{3}, 200 \mathrm{MHz}\right) \delta 0.93$ (bt, $\left.J=7.3 \mathrm{~Hz}, 3 \mathrm{H}\right), 1.43-1.58$ (m, $8 \mathrm{H}), 2.12$ (bt, $J=7.3 \mathrm{~Hz}, 2 \mathrm{H}), 3.38(\mathrm{~s}, 3 \mathrm{H}), 3.40(\mathrm{~s}, 3 \mathrm{H}), 5.66-6.00$ (m, 3H); ${ }^{13} \mathrm{C} \mathrm{NMR}$ $\left(\mathrm{CDCl}_{3}, 50.3 \mathrm{MHz}\right) \delta 175.4,152.8,139.5,130.6,130.2,121.5,106.4,97.4,51.6,51.2,36.9$, 27.1, 24.6, 20.6, 13.5; LSIMS $m / z$ (rel intensity) $305\left(\mathrm{MNa}^{+}, 70\right), 282\left(\mathrm{M}^{+}, 73\right), 239$ (54); HRMS (LSIMS) calcd for $\mathrm{C}_{15} \mathrm{H}_{22} \mathrm{O}_{5} 282.1467$, found 282.1465.

\section{3,3-dimethyl-1,4-dioxaspiro[4.5]deca-7,9-diene-6,6-dimethoxy-8-tert-}

butyldiphenylsilyloxymethyl-2-one (3c). Oxidation of the carboxylic acid 2c (164 mg, 0.34 mmol) was performed in $\mathrm{CH}_{3} \mathrm{CN} / \mathrm{MeOH}(9: 1)$ in the presence of 2,6-lutidine (2 equiv) according to the general procedure B. This electrolysis was carried out at a constant potential of $1.8 \mathrm{~V} / \mathrm{Ag} / 0.1 \mathrm{M} \mathrm{AgCl}$ until the current decayed smoothly to background with passage of 6.7 
F/mol. After processing of the reaction mixture, the residue was further dried overnight to give an oily crude product, which was purified by column chromatography, eluting $\mathrm{CH}_{2} \mathrm{Cl}_{2} /$ light petroleum $(2: 1)$, to furnish the spirolactone bisketal $\mathbf{3 c}$ as a pale yellow oil (107 $\mathrm{mg}, 62 \%)$; IR $(\mathrm{NaCl}) 1804,1730 \mathrm{~cm}^{-1} ;{ }^{1} \mathrm{H} \mathrm{NMR}\left(\mathrm{CDCl}_{3}, 250 \mathrm{MHz}\right) \delta 1.07(\mathrm{~s}, 9 \mathrm{H}), 1.52(\mathrm{~s}$, $3 \mathrm{H}), 1.62(\mathrm{~s}, 3 \mathrm{H}), 3.40(\mathrm{~s}, 3 \mathrm{H}), 3.43(\mathrm{~s}, 3 \mathrm{H}), 4.24(\mathrm{~s}, 2 \mathrm{H}), 5.85-6.11(\mathrm{~m}, 3 \mathrm{H}), 7.37-7.68(\mathrm{~m}$, $10 \mathrm{H}) ;{ }^{13} \mathrm{C} \mathrm{NMR}\left(\mathrm{CDCl}_{3}, 62.9 \mathrm{MHz}\right) \delta 175.4,138.6,135.4,133.0,129.9,127.8,127.5,120.2$, 106.5, 98.1, 64.3, 51.7, 51.4, 27.2, 26.7, 24.6, 19.2; EIMS $\mathrm{m} / z$ (rel intensity) $508\left(\mathrm{M}^{+}, 100\right)$, 407 (23), 239 (7), 137 (17); HRMS (EIMS) calcd for $\mathrm{C}_{29} \mathrm{H}_{36} \mathrm{SiO}_{6}$ 508.2281, found 508.2288.

\section{3,3-dimethyl-1,4-dioxaspiro[4.5]deca-7,9-diene-6,6-dimethoxy-9-tert-}

butyldiphenylsilyloxymethyl-2-one (3d). Oxidation of the propionic acid 2d (266 mg, 0.6 mmol) was performed in $\mathrm{CH}_{3} \mathrm{CN} / \mathrm{MeOH}(9: 1)$ in the presence of 2,6-lutidine (2 equiv) according to the general procedure B. This electrolysis was carried out at a constant potential of $1.8 \mathrm{~V} / \mathrm{Ag} / 0.1 \mathrm{M} \mathrm{AgCl}$ until the current decayed smoothly to background with passage of 4.1 $\mathrm{F} / \mathrm{mol}$. After processing of the reaction mixture, the residue was further dried overnight to give an oily crude product, which was purified by column chromatography, eluting with $\mathrm{CH}_{2} \mathrm{Cl}_{2} /$ light petroleum (2:1), to furnish the spirolactone bisketal 3d as a pale oil $(119 \mathrm{mg}$, 42\%); IR ( NaCl) 1791, $1265 \mathrm{~cm}^{-1} ;{ }^{1} \mathrm{H} \mathrm{NMR}\left(\mathrm{CDCl}_{3}, 250 \mathrm{MHz}\right) \delta 1.06$ (s, 9H), $1.52(\mathrm{~s}, 3 \mathrm{H})$, $1.61(\mathrm{~s}, 3 \mathrm{H}), 3.42(\mathrm{~s}, 3 \mathrm{H}), 3.43(\mathrm{~s}, 3 \mathrm{H}), 4.20(\mathrm{~s}, 2 \mathrm{H}), 5.91-6.05(\mathrm{~m}, 3 \mathrm{H}), 7.30-7.68(\mathrm{~m}, 10 \mathrm{H})$; ${ }^{13} \mathrm{C} \mathrm{NMR}\left(\mathrm{CDCl}_{3}, 62.9 \mathrm{MHz}\right) \delta 175.5,139.6,135.4,132.9,129.8,128.1,127.8,126.6,123.4$, 107.1, 97.7, 64.0, 51.7, 51.3, 27.1, 26.7, 24.6, 19.2; EIMS $\mathrm{m} / z$ (rel intensity) $508\left(\mathrm{M}^{+}, 100\right)$, 407 (29), 213 (50), 135 (85); HRMS (EIMS) calcd for $\mathrm{C}_{29} \mathrm{H}_{36} \mathrm{SiO}_{6}$ 508.2281, found 508.2288.

\section{8-bromo-3,3-dimethyl-1,4-dioxaspiro[4.5]deca-7,9-diene-6,6-dimethoxy-2-one}

(3e).

Oxidation of the propionic acid $2 \mathrm{e}(149 \mathrm{mg}, 0.51 \mathrm{mmol})$ was performed in $\mathrm{CH}_{3} \mathrm{CN} / \mathrm{MeOH}$ $(9: 1)$ in the presence of 2,6-lutidine ( 2 equiv) according to the general procedure B. This electrolysis was carried out at a constant potential of $1.8 \mathrm{~V} / \mathrm{Ag} / 0.1 \mathrm{M} \mathrm{AgCl}$ until the current decayed smoothly to background with passage of $4.1 \mathrm{~F} / \mathrm{mol}$. After processing of the reaction mixture, the residue was further dried overnight to give an oily crude product, which was 
purified by column chromatography, eluting with $\mathrm{CH}_{2} \mathrm{Cl}_{2}$, to furnish the spirolactone bisketal 3e as a pale yellow oil (115 mg, 70\%); IR (NaCl) $1805 \mathrm{~cm}^{-1} ;{ }^{1} \mathrm{H}$ NMR $\left(\mathrm{CDCl}_{3}, 250 \mathrm{MHz}\right) \delta$ $1.48(\mathrm{~s}, 3 \mathrm{H}), 1.56(\mathrm{~s}, 3 \mathrm{H}), 3.39(\mathrm{~s}, 3 \mathrm{H}), 3.41(\mathrm{~s}, 3 \mathrm{H}), 5.83$ (d, $J=10.1 \mathrm{~Hz}, 1 \mathrm{H}), 6.09$ (dd, $J=$ 2.0, $9.9 \mathrm{~Hz}, 1 \mathrm{H}), 6.33(\mathrm{~d}, J=1.8 \mathrm{~Hz}, 1 \mathrm{H}) ;{ }^{13} \mathrm{C} \mathrm{NMR}\left(\mathrm{CDCl}_{3}, 62.9 \mathrm{MHz}\right) \delta 174.8,131.8$, 131.6, 128.1, 120.2, 104.9, 98.6, 77.6, 51.8, 51.5, 27.0, 24.4; LSIMS m/z (rel intensity) 320 $\left(\mathrm{M}^{+}, 42\right), 318\left(\mathrm{M}^{+}, 42\right), 290$ (11), 289 (33), 288 (11), 287 (33), 239 (18); HRMS (LSIMS) calcd for $\mathrm{C}_{12} \mathrm{H}_{15} \mathrm{BrO}_{5}$ 318.0103, found 318.0104.

\section{9-bromo-3,3-dimethyl-1,4-dioxaspiro[4.5]deca-7,9-diene-6,6-dimethoxy-2-one}

Oxidation of the propionic acid $2 \mathrm{f}(147 \mathrm{mg}, 0.51 \mathrm{mmol})$ was performed in $\mathrm{CH}_{3} \mathrm{CN} / \mathrm{MeOH}$ (9:1) in the presence of 2,6-lutidine ( 2 equiv) according to the general procedure B. This electrolysis was carried out at a constant potential of $1.8 \mathrm{~V} / \mathrm{Ag} / 0.1 \mathrm{M} \mathrm{AgCl}$ until the current decayed smoothly to background with passage of $4.5 \mathrm{~F} / \mathrm{mol}$. After processing of the reaction mixture, the residue was further dried overnight to give an oily crude product, which was purified by column chromatography, eluting with $\mathrm{CH}_{2} \mathrm{Cl}_{2}$, to furnish the spirolactone bisketal 3e as a transparent oil (87 mg, 53\%); IR (NaCl) $1805 \mathrm{~cm}^{-1} ;{ }^{1} \mathrm{H}$ NMR $\left(\mathrm{CDCl}_{3}, 250 \mathrm{MHz}\right) \delta$ $1.51(\mathrm{~s}, 3 \mathrm{H}), 1.57(\mathrm{~s}, 3 \mathrm{H}), 3.41(\mathrm{~s}, 3 \mathrm{H}), 3.42(\mathrm{~s}, 3 \mathrm{H}), 5.96-6.18(\mathrm{~m}, 3 \mathrm{H}) ;{ }^{13} \mathrm{C} \mathrm{NMR}\left(\mathrm{CDCl}_{3}\right.$, $50.3 \mathrm{MHz}) \delta 174.7,130.8,130.5,129.2,121.6,106.7,99.0,96.8,51.8,51.4,27.0,24.4$. EIMS m/z (rel intensity) $320\left(\mathrm{M}^{+}, 7\right), 318$ (7), 234 (62), 232 (69), 289 (10), 287 (11), 219 (13), 217 (13); HRMS (EIMS) calcd for $\mathrm{C}_{12} \mathrm{H}_{15} \mathrm{BrO}_{5}$ 318.0103, found 318.0099.

\section{9-bromo-3,3-dimethyl-1,4-dioxaspiro[4.5]deca-7,9-diene-2,6-dione (4m). Selective} hydrolysis of spirolactone bisketal $\mathbf{3 f}(61 \mathrm{mg}, 0.19 \mathrm{mmol})$ in dry acetone $(7 \mathrm{~mL})$ was carried out at room temperature by treatment with $10 \%$ aq. $\mathrm{HCl}(10 \mathrm{~mL})$. The reaction mixture was stirred for 1 hour, after which time it was poured over saturated aq. $\mathrm{NaHCO}_{3}$ and extracted with $\mathrm{Et}_{2} \mathrm{O}(3 \times 10 \mathrm{~mL})$. The combined extracts were dried over $\mathrm{Na}_{2} \mathrm{SO}_{4}$, filtered, evaporated to give a residue, which was submitted to column chromatography, eluting with $\mathrm{CH}_{2} \mathrm{Cl}_{2}$, to give $\mathbf{4 m}$ as a yellow oil (37 $\mathrm{mg}, 71 \%)$. 
This orthoquinone monoketal was also prepared by oxidation of the propionic acid $\mathbf{2 f}$ (121 $\mathrm{mg}, 0.42 \mathrm{mmol}$ ) performed in $\mathrm{CH}_{3} \mathrm{CN} / \mathrm{H}_{2} \mathrm{O}$ (9:1) in the presence of 2,6-lutidine (2 equiv) according to the general electrochemical procedure B. This electrolysis was carried out at a constant potential of $1.8 \mathrm{~V} / \mathrm{Ag} / 0.1 \mathrm{M} \mathrm{AgCl}$ until the current decayed smoothly to background with passage of $4.1 \mathrm{~F} / \mathrm{mol}$. After processing of the reaction mixture, the resulting oil was purified by column chromatography, eluting with $\mathrm{CH}_{2} \mathrm{Cl}_{2}$, to furnish $\mathbf{4 m}$ as a yellow oil (59 mg, 52\%); IR ( NaCl) $1814 \mathrm{~cm}^{-1} ;{ }^{1} \mathrm{H} \mathrm{NMR}\left(\mathrm{CDCl}_{3}, 300 \mathrm{MHz}\right) \delta 1.53,(\mathrm{~s}, 6 \mathrm{H}), 1.63(\mathrm{~s}, 6 \mathrm{H})$, $6.05(\mathrm{~d}, J=10.3 \mathrm{~Hz}, 1 \mathrm{H}), 6.49(\mathrm{~d}, J=2.3 \mathrm{~Hz}, 1 \mathrm{H}), 6.97(\mathrm{dd}, J=2.3,10.3 \mathrm{~Hz}, 1 \mathrm{H}) ;{ }^{13} \mathrm{C} \mathrm{NMR}$ $\left(\mathrm{CDCl}_{3}, 50.3 \mathrm{MHz}\right) \delta 191.9,174.2,144.4,134.9,125.0,122.4,94.4,78.3,27.4,25.5$; EIMS $m / z$ (rel intensity) $274\left(\mathrm{M}^{+}, 13\right), 272\left(\mathrm{M}^{+}, 10\right), 193$ (16), 188 (13), 186 (10), 102 (100).

Tricyclododecadienedione 5i. Selective hydrolysis of spirolactone bisketal $\mathbf{3 b}(80 \mathrm{mg}, 0.3$ mmol) in dry acetone $(4 \mathrm{~mL})$ was carried out at room temperature by treatment with $2 \mathrm{~N}$ $\mathrm{H}_{2} \mathrm{SO}_{4}(8 \mathrm{~mL})$. The reaction mixture was stirred for $30 \mathrm{~min}$, poured over a $5 \% \mathrm{NaHCO}_{3}$ aq. solution, extracted with $\mathrm{Et}_{2} \mathrm{O}(2 \times 10 \mathrm{~mL})$, dried over $\mathrm{Na}_{2} \mathrm{SO}_{4}$, filtered, and evaporated to give 5i as a pale yellow oil (52 $\mathrm{mg}, 78 \%)$.

This dimer was also prepared by oxidation of the propionic acid $\mathbf{2 b}$ (400 mg, $1.57 \mathrm{mmol}$ ) performed in $\mathrm{CH}_{3} \mathrm{CN} / \mathrm{H}_{2} \mathrm{O}(9: 1)$ in the presence of pyridine (2 equiv) according to the general electrochemical procedure B. This electrolysis was carried out at a constant potential of $1.5 \mathrm{~V} / \mathrm{Ag} / 0.1 \mathrm{M} \mathrm{AgCl}$ until the current decayed smoothly to background. After processing of the reaction mixture, the resulting brown oil was purified by column chromatography, eluting with light petroleum/ $\mathrm{Et}_{2} \mathrm{O}(4: 1 \rightarrow 1: 1)$, to furnish $\mathbf{5 i}$ as a pale yellow oil $(115 \mathrm{mg}, 31 \%)$; IR $(\mathrm{NaCl}) 2963,2935,1811,1749,1702 \mathrm{~cm}^{-1} ;{ }^{1} \mathrm{H} \mathrm{NMR}\left(\mathrm{CDCl}_{3}, 250 \mathrm{MHz}\right) \delta 0.82$ (bt, $J=7.3$ $\mathrm{Hz}, 3 \mathrm{H}), 0.99$ (bt, $J=7.3 \mathrm{~Hz}, 3 \mathrm{H}), 1.29-1.62$ (m, 16H), 1.85-1.94 (m, 2H), 2.17-2.29 (m, 2H), 3.04-3.31 (m, 4H), $5.95(\mathrm{~d}, J=7.3 \mathrm{~Hz}, 1 \mathrm{H}), 6.07(\mathrm{~s}, 1 \mathrm{H}) ;{ }^{13} \mathrm{C} \mathrm{NMR}\left(\mathrm{CDCl}_{3}, 75.5 \mathrm{MHz}\right) \delta$ 199.5, 188.9, 174.5, 160.9, 147.8, 125.9, 123.3, 101.3, 98.9, 78.8, 78.2, 55.5, 43.8, 42.2, 40.4, 37.4, 37.3, 27.0, 26.8, 25.4, 24.0, 20.8, 19.4, 13.5; LSIMS $m / z$ (rel intensity) $495\left(\mathrm{MNa}^{+}, 47\right)$, 473( $\left.\mathrm{MH}^{+}, 13\right), 444$ (12), 409(68); HRMS (LSIMS) calcd for $\mathrm{C}_{26} \mathrm{H}_{32} \mathrm{O}_{8} \mathrm{Na}$ 495.1995, found 495.1988 . 
Tricyclododecadienedione 5l. Selective hydrolysis of spirolactone bisketal 3e (105 mg, 0.3 $\mathrm{mmol})$ in dry acetone $(15 \mathrm{~mL})$ was carried out at room temperature by treatment with $10 \%$ aq. $\mathrm{HCl}(5 \mathrm{~mL})$. The reaction mixture was stirred for $1 \mathrm{~h}$, poured over a $5 \% \mathrm{NaHCO}_{3}$ aq. solution, extracted with $\mathrm{Et}_{2} \mathrm{O}(3 \times 20 \mathrm{~mL})$, dried over $\mathrm{Na}_{2} \mathrm{SO}_{4}$, filtered, and evaporated to give crude $\mathbf{5 l}$, which was submitted to column chromatography, eluting with $\mathrm{CH}_{2} \mathrm{Cl}_{2}$, to yield pure $\mathbf{5 l}$ as an off-white solid (70 mg, 78\%).

This dimer was also prepared by oxidation of the propionic acid $2 \mathrm{e}(103 \mathrm{mg}, 0.35 \mathrm{mmol})$ performed in $\mathrm{CH}_{3} \mathrm{CN} / \mathrm{H}_{2} \mathrm{O}$ (9:1) in the presence of 2,6-lutidine (2 equiv) according to the general electrochemical procedure B. This electrolysis was carried out at a constant potential of $1.8 \mathrm{~V} / \mathrm{Ag} / 0.1 \mathrm{M} \mathrm{AgCl}$ until the current decayed smoothly to background with passage of 5 $\mathrm{F} / \mathrm{mol}$. After processing of the reaction mixture, the resulting brown oil was purified by column chromatography, eluting with $\mathrm{CH}_{2} \mathrm{Cl}_{2}$, to furnish an amorphous pale yellow solid, which was crystallized from $\mathrm{CH}_{2} \mathrm{Cl}_{2}$ to afford dimer $\mathbf{5 l}$ as an off-white solid (23.4 $\mathrm{mg}, 24 \%$ ), mp 251-252 ${ }^{\circ} \mathrm{C}$; IR (NaCl) 1821, 1760, $1720 \mathrm{~cm}^{-1} ;{ }^{1} \mathrm{H}$ NMR $\left(\mathrm{CDCl}_{3}, 250 \mathrm{MHz}\right) \delta 1.33(\mathrm{~s}$, $3 \mathrm{H}), 1.53(\mathrm{~s}, 3 \mathrm{H}), 1.56(\mathrm{~s}, 3 \mathrm{H}), 1.59(\mathrm{~s}, 3 \mathrm{H}), 3.02(\mathrm{dd}, J=2.5,8.5 \mathrm{~Hz}, 1 \mathrm{H}), 3.38(\mathrm{dd}, J=7.4$, $1.3 \mathrm{~Hz}, 1 \mathrm{H}), 3.79(\mathrm{dd}, J=8.1,2.4 \mathrm{~Hz}, 1 \mathrm{H}), 4.06(\mathrm{t}, J=2.4 \mathrm{~Hz}, 1 \mathrm{H}), 6.52(\mathrm{dd}, J=7.0,1.8 \mathrm{~Hz}$, 1H), $6.76(\mathrm{~s}, 1 \mathrm{H}) ;{ }^{13} \mathrm{C} \mathrm{NMR}\left(\mathrm{CDCl}_{3}, 62.9 \mathrm{MHz}\right) \delta 196.8,185.8,173.6,144.3,133.9,130.0$, $118.4,100.2,97.8,79.3,78.7,60.5,47.3,46.0,42.8,29.7,26.9,26.6,25.3,24.0$; LSIMS $m / z$ (rel intensity) $549\left(\mathrm{MH}^{+}, 4\right), 547\left(\mathrm{MH}^{+}, 8\right), 545\left(\mathrm{MH}^{+}, 4\right), 462$ (12), 460 (21), 458 (12), 273 (11), 213 (100); HRMS (LSIMS) calcd for $\mathrm{C}_{20} \mathrm{H}_{19} \mathrm{Br}_{2} \mathrm{O}_{8} 546.9426$, found 546.9405.

Pentacyclododecanedione 6a. A solution of $5 \mathrm{a}(70.7 \mathrm{mg}, 0.23 \mathrm{mmol})$ in $\mathrm{CH}_{2} \mathrm{Cl}_{2}(2 \mathrm{~mL})$ in a Pyrex tube was irradiated at room temperature for $20 \mathrm{~h}$ using a medium-pressure mercury lamp. This reaction mixture was then evaporated to furnish 6a as a brown oil (68.2.mg, 96\%); IR (NaCl) 2990, 2950, 1727, $1262 \mathrm{~cm}^{-1} ;{ }^{1} \mathrm{H}$ NMR $\left(\mathrm{CDCl}_{3}, 300 \mathrm{MHz}\right) \delta 2.87$ (bs, 2H), 3.07$3.16(\mathrm{~m}, 6 \mathrm{H}), 3.24(\mathrm{~s}, 6 \mathrm{H}), 3.38(\mathrm{~s}, 6 \mathrm{H}) ;{ }^{13} \mathrm{C} \mathrm{NMR}\left(\mathrm{CDCl}_{3}, 62.9 \mathrm{MHz}\right) \delta 203.8,95.2,50.3$, 49.2, 46.2, 36.7, 36.6, 35.6; EIMS $m / z$ (rel intensity) $308\left(\mathrm{M}^{+}, 8\right), 280$ (50), 237 (79), 205 (87), 159 (100), 103 (97); HRMS (ESIMS) calcd for $\mathrm{C}_{16} \mathrm{H}_{20} \mathrm{O}_{6} \mathrm{Na} 331.1158$, found 331.1158. 
Pentacyclododecanedione 6b. A solution of $\mathbf{5 b}(69 \mathrm{mg}, 0.083 \mathrm{mmol})$ in $\mathrm{CH}_{2} \mathrm{Cl}_{2}(2 \mathrm{~mL})$ in a Pyrex tube was irradiated at room temperature for $15 \mathrm{~h}$ using a medium-pressure mercury lamp. This reaction mixture was then evaporated to furnish $\mathbf{6 b}$ as a yellow oil (54.mg, 78\%); IR (NaCl) 2934, 2852, $1728 \mathrm{~cm}^{-1} ;{ }^{1} \mathrm{H}$ NMR $\left(\mathrm{CDCl}_{3}, 250 \mathrm{MHz}\right) 1.02$ (s, 18H), 2.90 (bs, 2H), 3.08 (bs, 10H), 3.37 (s, 6H), 3.71 (d, $J=11.0 \mathrm{~Hz}, 2 \mathrm{H}), 3.89$ (d, $J=11.0 \mathrm{~Hz}, 2 \mathrm{H}), 7.30-7.74$ $(\mathrm{m}, 20 \mathrm{H}) ;{ }^{13} \mathrm{C} \mathrm{NMR}\left(\mathrm{CDCl}_{3}, 75.5 \mathrm{MHz}\right) \delta 203.9,135.5,132.8,129.7,127.7,95.7,63.8,50.2$, 49.2, 48.2, 47.1, 37.3, 36.7, 26.8, 19.2; ESIMS $m / z$ (rel intensity) $844\left(\mathrm{M}^{+}, 21\right)$; HRMS (ESIMS) calcd for $\mathrm{C}_{50} \mathrm{H}_{60} \mathrm{O}_{8} \mathrm{Si}_{2} \mathrm{Na}$ 867.3724, found 867.3723.

Pentacyclododecanedione 6c. A solution of 5c (17 mg, $0.037 \mathrm{mmol})$ in $\mathrm{CH}_{2} \mathrm{Cl}_{2}(2 \mathrm{~mL})$ was irradiated in a Pyrex tube at room temperature for $9 \mathrm{~h}$ using a medium-pressure mercury lamp. This reaction mixture was then evaporated to furnish 6a as a brown oil (16.mg, 94\%); IR $(\mathrm{NaCl}) 2945,2847,1732 \mathrm{~cm}^{-1} ;{ }^{1} \mathrm{H}$ NMR $\left(\mathrm{CDCl}_{3}, 250 \mathrm{MHz}\right) \delta 3.22-3.40(\mathrm{~m}, 2 \mathrm{H}), 3.29(\mathrm{~s}, 6 \mathrm{H})$, $3.37(\mathrm{~s}, 6 \mathrm{H}), 3.41-3.72(\mathrm{~m}, 4 \mathrm{H}) ;{ }^{13} \mathrm{C} \mathrm{NMR}\left(\mathrm{CDCl}_{3}, 50.3 \mathrm{MHz}\right) \delta$ 198.3, 95.4, 57.8, 54.1, 50.8, 49.1, 48.2, 37.1 ; CIMS $m / z$ (rel intensity) $486\left(\mathrm{MNH}_{4}{ }^{+}, 97\right), 484\left(\mathrm{MNH}_{4}{ }^{+}, 100\right), 482$ $\left(\mathrm{MNH}_{4}{ }^{+}, 38\right)$; HRMS (ESIMS) calcd for $\mathrm{C}_{16} \mathrm{H}_{18} \mathrm{O}_{6} \mathrm{Br}_{2} \mathrm{Na} 486.9368$, found 486.9359.

Pentacyclododecanedione 6i. A solution of $5 \mathbf{i}(12.7 \mathrm{mg}, 0.027 \mathrm{mmol})$ in a hexane- $\mathrm{Et}_{2} \mathrm{O}(3: 1)$ mixture $(4 \mathrm{~mL})$ was irradiated in a Pyrex tube at room temperature for $15 \mathrm{~h}$ using a medium pressure mercury lamp. This reaction mixture was then evaporated to furnish $\mathbf{6} \mathbf{i}$ as a white powder $(11 \mathrm{mg}, 83 \%)$. Crystallization of this powder from $\mathrm{CH}_{2} \mathrm{Cl}_{2}$ gave $\mathbf{6 i}$ as pale yellow crystals, mp $182{ }^{\circ} \mathrm{C}$; IR $(\mathrm{NaCl}) 1811,1749,1702 \mathrm{~cm}^{-1} ;{ }^{1} \mathrm{H}$ NMR $\left(\mathrm{CDCl}_{3}, 300 \mathrm{MHz}\right) \delta 0.88-$ $1.06(\mathrm{~m}, 6 \mathrm{H}), 1.09-1.25(\mathrm{~m}, 4 \mathrm{H}), 1.27-1.29(\mathrm{~m}, 4 \mathrm{H}), 1.49(\mathrm{~s}, 6 \mathrm{H}), 1.52(\mathrm{~s}, 6 \mathrm{H}), 2.80(\mathrm{bs}, 2 \mathrm{H})$, 3.04 (s, 4H); ${ }^{13} \mathrm{C} \mathrm{NMR}\left(\mathrm{CDCl}_{3}, 62.9 \mathrm{MHz}\right) \delta 203.1,174.2,99.4,78.8,48.3,47.8,41.0,37.7$, 33.4, 27.1, 24.9, 16.8, 14.5; LSIMS $m / z$ (rel intensity) $495\left(\mathrm{MNa}^{+}, 68\right), 409$ (100); HRMS (LSIMS) calcd for $\mathrm{C}_{26} \mathrm{H}_{32} \mathrm{O}_{8} \mathrm{Na} 495.1995$, found 495.1990 .

X-ray Analysis of 6i. Crystals of $\mathbf{6} \mathbf{i}$ were obtained in $\mathrm{CH}_{2} \mathrm{Cl}_{2}$ by slow evaporation. A crystal of $0.12 \times 0.25 \times 0.25 \mathrm{~mm}$ was chosen for structural determination. X-ray diffraction data were 
collected using a CAD4 Enraf-Nonius diffractometer with graphite monochromatized CuK $\alpha$ radiation. The crystal is orthorhombic, space group P b c a, with a=11.075(1) $\AA, b=18.989(3)$ $\AA, c=23.195(2) \AA$, and $Z=4$. The cell parameters were determined by least-squares from the setting angles for 25 reflexions. An empirical absorption correction was applied. The data were also corrected for Lorentz and polarization effect. The positions of non-H atoms were determined by the program SHELXS 86 and the position of the $\mathrm{H}$ atoms were deduced from coordinates of the non-H atoms and confirmed by Fourier Synthesis. $\mathrm{H}$ atoms were included for structure factor calculations but not refined. The reliability factor was $\mathrm{R}=4.82 \%$ for 4146 reflections.

\section{ORTEP diagram of the X-ray structure of $6 i$}

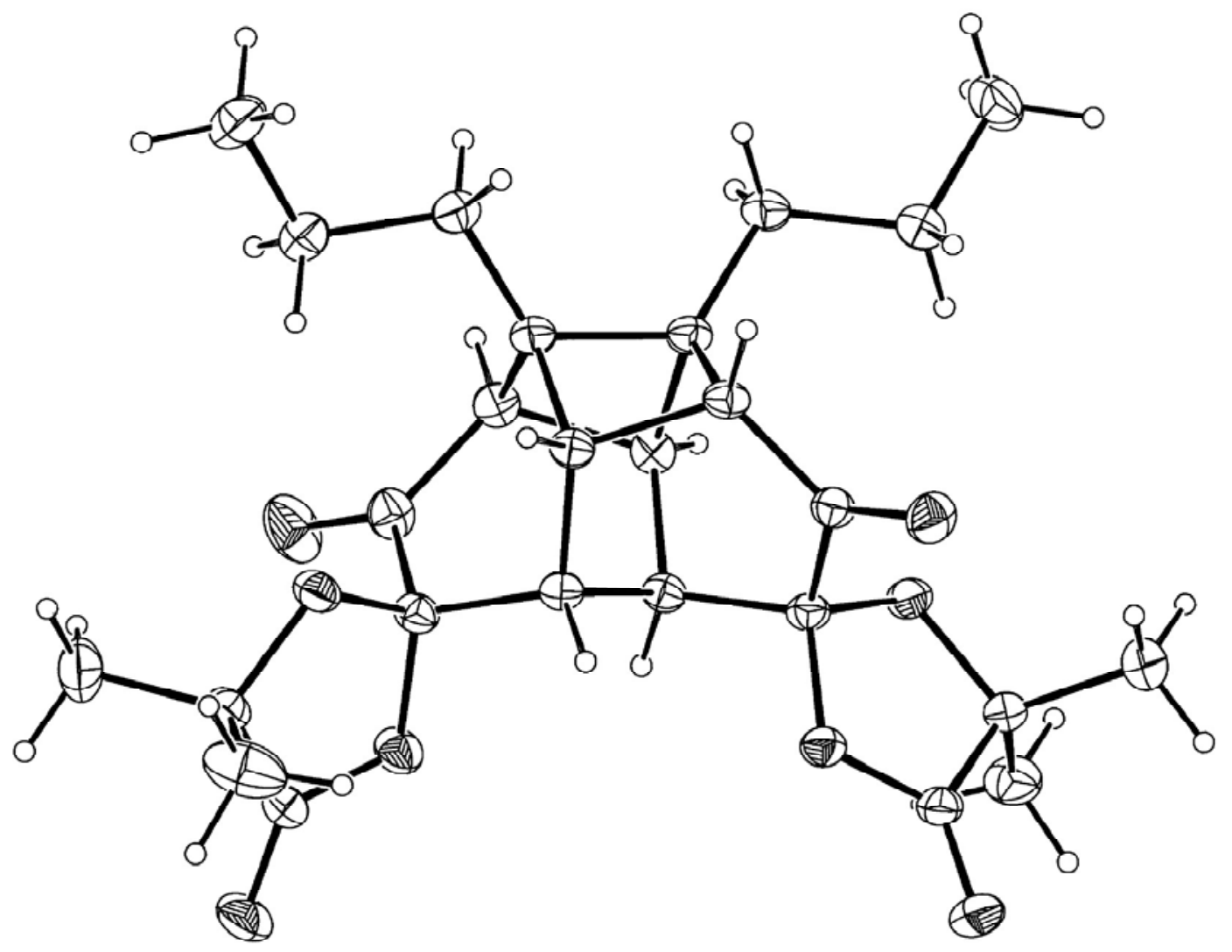


Calculations. Semiempirical and ab initio RHF/3-21G methods were used to calculate the relative magnitudes of atomic coefficients $\left(C_{i}\right.$, Table 1) and the energy levels (Table 2) of HOMOs and LUMOs of orthoquinone monoketals 4a,c,e,f. The geometries were fully optimized by PM3 semiempirial methods and used as the basis for optimization at the HarteeFock level using the 3-21G basis set.

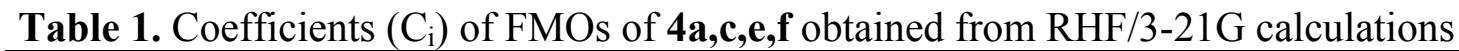

\begin{tabular}{lllllllllll}
\hline Compound & \multicolumn{4}{c}{ HOMO } & \multicolumn{5}{c}{ LUMO } \\
\cline { 2 - 11 } & $\mathrm{C}_{2}$ & $\mathrm{C}_{3}$ & $\mathrm{C}_{4}$ & $\mathrm{C}_{5}$ & $\Delta \mathrm{C}_{\mathrm{i}}$ & $\mathrm{C}_{2}$ & $\mathrm{C}_{3}$ & $\mathrm{C}_{4}$ & $\mathrm{C}_{5}$ & $\Delta \mathrm{C}_{\mathrm{i}}$ \\
\hline $\mathbf{4 a}$ & 0.259 & 0.183 & -0.202 & -0.250 & 0.168 & -0.220 & -0.122 & 0.193 & \\
$\mathbf{4 a}^{1}$ & 0.260 & 0.197 & -0.196 & -0.252 & 0.177 & -0.210 & -0.140 & 0.192 & \\
$\mathbf{4 a}^{2}$ & 0.260 & 0.185 & -0.294 & -0.247 & -0.168 & 0.223 & 0.123 & -0.192 & \\
$\mathbf{4 c}$ & -0.216 & -0.146 & 0.207 & 0.261 & -0.172 & 0.220 & 0.131 & -0.194 & \\
$\mathbf{4 e}$ & -0.270 & -0.187 & 0.168 & 0.212 & 0.170 & -0.234 & -0.121 & 0.194 & \\
$\mathbf{4 f}$ & -0.246 & -0.136 & 0.243 & 0.231 & -0.151 & 0.231 & 0.093 & -0.211 \\
\hline
\end{tabular}

${ }^{1}$ Calculations performed at the same level by Liao et al.[Ref. 14b]

${ }^{2}$ Calculations performed at the ab Initio HF/6-31G* level by Tamariz et al.[Ref. 14c]

Table 2. Energies of FMOs of $\mathbf{4 a}, \mathbf{c}, \mathbf{e}, \mathbf{f}$ obtained from semiempirical PM3 and ab initio RHF/3-21G calculations

\begin{tabular}{lccccc}
\hline Compd & FMO & PM3 $(\mathrm{eV})$ & $\Delta(\mathrm{H}-\mathrm{L})^{1}(\mathrm{eV})$ & $3-21 \mathrm{G}(\mathrm{eV})$ & $\Delta(\mathrm{H}-\mathrm{L})^{1}(\mathrm{eV})$ \\
\hline $\mathbf{4 a}$ & HOMO & -9.897 & - & -9.336 & - \\
& LUMO & -0.934 & 8.963 & 1.605 & 10.941 \\
$\mathbf{4 a}^{2}$ & HOMO & -10.043 & - & -9.333 & - \\
& LUMO & -0.950 & 9.093 & 1.725 & 11.058 \\
$\mathbf{4 a}^{3}$ & HOMO & - & - & $-9.519^{4}$ & - \\
& LUMO & - & - & $1.442^{4}$ & $10.961^{4}$ \\
$\mathbf{4 c}$ & HOMO & -10.129 & - & -9.492 & - \\
& LUMO & -1.219 & 8.910 & 1.281 & 10.773 \\
$\mathbf{4 e}$ & HOMO & -10.144 & - & -9.524 & \\
& LUMO & -1.248 & 8.896 & 1.271 & 10.795 \\
$\mathbf{4 f}$ & HOMO & -9.535 & & -8.648 & \\
& LUMO & -0.968 & 8.567 & 1.763 & 10.411 \\
\hline
\end{tabular}

Tenergy gap (HOMO-LUMO)

${ }^{2}$ Calculations performed at the same level by Liao et al.[Ref. 14b]

${ }^{3}$ Calculations performed at the ab Initio HF/6-31G* level by Tamariz et al.[Ref. 14c]

SPARTAN AB INITIO PROGRAM: SGI/R10K/32 Release 5.1.3

\section{Comound 4a}

Run type: Geometry optimization

Model: RHF/3-21G

Number of shells: 53

$31 \mathrm{~S}$ shells

22 SP shells 
Number of basis functions: 119

Number of electrons: 82

Use of molecular symmetry disabled

Molecular charge: 0

Spin multiplicity: 1

\begin{tabular}{lrrrr}
\multicolumn{5}{c}{ Cartesian Coordinates (Angstroms) } \\
Atom Label & X & \multicolumn{1}{c}{ Y } & \multicolumn{1}{c}{ Z } \\
\hline--------- & \\
H H17 & -2.2966262 & 0.3855762 & 0.5868297 \\
H H1 & -1.0845049 & -0.6521514 & -3.4107245 \\
C C2 & -1.7914114 & -0.0527448 & -1.4320959 \\
H H9 & -2.8130192 & 0.0655526 & -1.8109779 \\
C C1 & -1.5131700 & 0.1286421 & -0.1370176 \\
C C3 & -0.1186374 & -0.0604230 & 0.4347447 \\
O O2 & -0.2077038 & -1.2271085 & 1.2338219 \\
O O3 & 0.1602800 & 0.8630311 & 1.4739362 \\
C C4 & 0.9757630 & -0.1112975 & -0.6747757 \\
O O1 & 2.1458068 & 0.1139994 & -0.4354907 \\
C C5 & 0.5308089 & -0.4500665 & -2.0453788 \\
C C6 & -0.7632817 & -0.4138395 & -2.3903063 \\
H H8 & 1.3109980 & -0.7083611 & -2.7717845 \\
C C8 & 0.9904418 & -1.8203568 & 1.6697882 \\
C C7 & 0.1615373 & 2.2210076 & 1.1220042 \\
H H2 & 0.3703277 & 2.7206757 & 2.0710252 \\
H H3 & -0.8120312 & 2.5416988 & 0.7323612 \\
H H4 & 0.9433295 & 2.4589054 & 0.3920110 \\
H H5 & 0.6234523 & -2.6047451 & 2.3371655 \\
H H6 & 1.6424538 & -1.1327352 & 2.2189954 \\
H H7 & 1.5451868 & -2.2652595 & 0.8358687
\end{tabular}

Point Group $=\mathrm{C} 1$ Order $=1$ Nsymop $=1$

This system has 57 degrees of freedom

Coordinates read from pre-optimization

\begin{tabular}{|c|c|c|c|}
\hline \multirow[b]{2}{*}{ Atom Label } & \multicolumn{3}{|c|}{ Cartesian Coordinates (Angstroms) } \\
\hline & $\mathrm{X}$ & $\mathrm{Y} \quad \mathrm{Z}$ & \\
\hline H H17 & -4.3399963 & 0.7286338 & 1.1089479 \\
\hline H H1 & -2.0494181 & -1.2323880 & -6.4453377 \\
\hline $\mathrm{C}$ C2 & -3.3852782 & -0.0996732 & -2.7062702 \\
\hline H H9 & -5.3158379 & 0.1238766 & -3.4222536 \\
\hline $\mathrm{C}$ C1 & -2.8594781 & 0.2430984 & -0.2589259 \\
\hline $\mathrm{C}$ C3 & -0.2241923 & -0.1141830 & 0.8215487 \\
\hline $\mathrm{O} \mathrm{O} 2$ & -0.3925035 & -2.3188999 & 2.3315865 \\
\hline $\mathrm{OO} 3$ & 0.3028855 & 1.6308930 & 2.7853369 \\
\hline $\mathrm{C}$ C4 & 1.8439255 & -0.2103218 & -1.2751417 \\
\hline $\mathrm{O} \mathrm{O} 1$ & 4.0549888 & 0.2154278 & -0.8229585 \\
\hline
\end{tabular}




$\begin{array}{lrrr}\text { C C5 } & 1.0030839 & -0.8505028 & -3.8652072 \\ \text { C C6 } & -1.4423940 & -0.7820437 & -4.5170261 \\ \text { H H8 } & 2.4774282 & -1.3386091 & -5.2379156 \\ \text { C C8 } & 1.8716645 & -3.4399771 & 3.1554437 \\ \text { C C7 } & 0.3052613 & 4.1970978 & 2.1202814 \\ \text { H H2 } & 0.6998182 & 5.1413340 & 3.9136719 \\ \text { H H3 } & -1.5345171 & 4.8031166 & 1.3839627 \\ \text { H H4 } & 1.7826351 & 4.6466597 & 0.7407937 \\ \text { H H5 } & 1.1781546 & -4.9222569 & 4.4166044 \\ \text { H H6 } & 3.1037891 & -2.1405602 & 4.1932952 \\ \text { H H7 } & 2.9199811 & -4.2807217 & 1.5795635\end{array}$

Initial Hessian option

Hessian from PM3D calculation used.

Max. Max. Neg.

Cycle Energy Grad. Dist. Eigen

$\begin{array}{llll}1 & -530.3016430 & 0.03557 & 0.01364\end{array}$

$\begin{array}{llll}2 & -530.3137701 & 0.01658 & 0.02591\end{array}$

$\begin{array}{llll}3 & -530.3181322 & 0.00673 & 0.02486\end{array}$

$\begin{array}{llll}4 & -530.3191261 & 0.00719 & 0.04061\end{array}$

$\begin{array}{llll}5 & -530.3194902 & 0.00453 & 0.04064\end{array}$

$\begin{array}{llll}6 & -530.3198806 & 0.00309 & 0.04100\end{array}$

$\begin{array}{lllll}7 & -530.3201388 & 0.00504 & 0.03305\end{array}$

$\begin{array}{llll}8 & -530.3203265 & 0.00450 & 0.03876\end{array}$

$\begin{array}{lllll}9 & -530.3205430 & 0.00284 & 0.02650\end{array}$

$\begin{array}{lllll}10 & -530.3206742 & 0.00402 & 0.02299\end{array}$

$\begin{array}{lllll}11 & -530.3206197 & 0.00392 & 0.01234\end{array}$

$\begin{array}{lllll}12 & -530.3208006 & 0.00233 & 0.02270\end{array}$

$\begin{array}{lllll}13 & -530.3206216 & 0.00838 & 0.01509\end{array}$

$\begin{array}{lllll}14 & -530.3207816 & 0.00402 & 0.01857\end{array}$

$\begin{array}{lllll}15 & -530.3206891 & 0.00579 & 0.00835\end{array}$

$\begin{array}{lllll}16 & -530.3208850 & 0.00081 & 0.00786\end{array}$

$\begin{array}{lllll}17 & -530.3208890 & 0.00214 & 0.00062\end{array}$

$\begin{array}{lllll}18 & -530.3209148 & 0.00113 & 0.00095\end{array}$

$\begin{array}{lllll}19 & -530.3209290 & 0.00071 & 0.00158\end{array}$

$\begin{array}{lllll}20 & -530.3209423 & 0.00068 & 0.00291\end{array}$

$\begin{array}{lllll}21 & -530.3209586 & 0.00108 & 0.00508\end{array}$

$\begin{array}{lllll}22 & -530.3209752 & 0.00093 & 0.00885\end{array}$

$\begin{array}{lllll}23 & -530.3209973 & 0.00117 & 0.01167\end{array}$

$\begin{array}{lllll}24 & -530.3210221 & 0.00136 & 0.01253\end{array}$

$\begin{array}{lllll}25 & -530.3210749 & 0.00082 & 0.02230\end{array}$

$\begin{array}{lllll}26 & -530.3210796 & 0.00361 & 0.00829\end{array}$

$\begin{array}{lllll}27 & -530.3211585 & 0.00218 & 0.01344\end{array}$

$\begin{array}{lllll}28 & -530.3212019 & 0.00071 & 0.02281\end{array}$

$\begin{array}{lllll}29 & -530.3212156 & 0.00219 & 0.00082\end{array}$

$\begin{array}{lllll}30 & -530.3212282 & 0.00098 & 0.00054\end{array}$

$\begin{array}{lllll}31 & -530.3212310 & 0.00070 & 0.00070\end{array}$

$\begin{array}{lllll}32 & -530.3212345 & 0.00059 & 0.00024\end{array}$

$\begin{array}{lllll}33 & -530.3212373 & 0.00062 & 0.00047\end{array}$

$\begin{array}{lllll}34 & -530.3212408 & 0.00033 & 0.00084\end{array}$ 


$$
\begin{array}{llll}
35 & -530.3212438 & 0.00037 & 0.00173 \\
36 & -530.3212470 & 0.00033 & 0.00141 \\
37 & -530.3212482 & 0.00035 & 0.00008 \\
38 & -530.3212489 & 0.00013 & 0.00006
\end{array}
$$

\begin{tabular}{|c|c|c|c|}
\hline \multirow[b]{2}{*}{ Atom Label } & \multicolumn{3}{|c|}{ Cartesian Coordinates (Angstroms) } \\
\hline & X & $\begin{array}{ll}Y & Z\end{array}$ & \\
\hline H H17 & -2.2898642 & 0.1547730 & 0.6564373 \\
\hline H H1 & -1.1759661 & -0.3858454 & -3.4351843 \\
\hline C C2 & -1.8803238 & -0.0512694 & -1.3935382 \\
\hline H H9 & -2.8981822 & 0.0356101 & -1.7167448 \\
\hline $\mathrm{C} \mathrm{C} 1$ & -1.5612762 & 0.0231810 & -0.1165981 \\
\hline $\mathrm{C} \mathrm{C} 3$ & -0.1523819 & -0.1599249 & 0.3769329 \\
\hline $\mathrm{O} \mathrm{O} 2$ & -0.1326285 & -1.4565667 & 0.9563960 \\
\hline $\mathrm{OO} \mathrm{O}$ & 0.1577739 & 0.7708412 & 1.3954345 \\
\hline $\mathrm{C}$ C4 & 0.9057974 & -0.1475046 & -0.7380330 \\
\hline $\mathrm{OO} \mathrm{O}$ & 2.0851762 & -0.0252988 & -0.4670822 \\
\hline $\mathrm{C} \mathrm{C5}$ & 0.4387662 & -0.3370335 & -2.1176682 \\
\hline C C6 & -0.8500808 & -0.2768348 & -2.4194313 \\
\hline H H8 & 1.1961606 & -0.4792857 & -2.8602005 \\
\hline $\mathrm{C} \mathrm{C} 8$ & 0.9665432 & -1.7552503 & 1.8626393 \\
\hline $\mathrm{C} \mathrm{C7}$ & 0.3472995 & 2.1470375 & 1.0023633 \\
\hline $\mathrm{H} \mathrm{H} 2$ & 0.4481230 & 2.7059340 & 1.9186067 \\
\hline $\mathrm{H} \mathrm{H} 3$ & -0.5073654 & 2.5156275 & 0.4471568 \\
\hline H H4 & 1.2426731 & 2.2567791 & 0.4092238 \\
\hline H H5 & 0.8519984 & -2.8015487 & 2.0977365 \\
\hline H H6 & 0.8881755 & -1.1543606 & 2.7526632 \\
\hline H H7 & 1.9195820 & -1.5790598 & 1.3888903 \\
\hline
\end{tabular}

Compound 4c

Run type: Geometry optimization

Model: RHF/3-21G

Number of shells: 57

$30 \mathrm{~S}$ shells

26 SP shells

$16 \mathrm{D}$ shells

Number of basis functions: 140

Number of electrons: 116

Use of molecular symmetry disabled

Molecular charge: 0

Spin multiplicity: 1

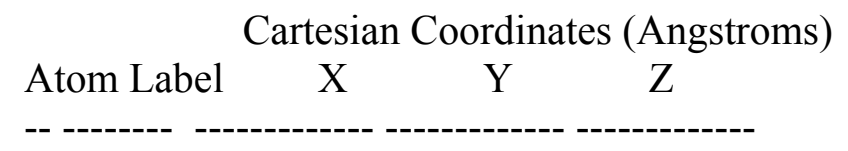




$\begin{array}{lrrr}\text { H H17 } & -2.2912258 & 0.3426612 & 0.6044161 \\ \text { H H1 } & -1.0891060 & -0.5836128 & -3.4162451 \\ \text { C C2 } & -1.7204232 & -0.0589743 & -1.4018733 \\ \text { Br Br1 } & -3.4612911 & 0.1392191 & -2.0576220 \\ \text { C C1 } & -1.4881523 & 0.1077359 & -0.1046715 \\ \text { C C3 } & -0.0847882 & -0.0767126 & 0.4553400 \\ \text { O O2 } & -0.1685375 & -1.2395166 & 1.2585266 \\ \text { O O3 } & 0.2030753 & 0.8551837 & 1.4819460 \\ \text { C C4 } & 1.0024978 & -0.1321547 & -0.6686323 \\ \text { O O1 } & 2.1764522 & 0.0641397 & -0.4254379 \\ \text { C C5 } & 0.5585989 & -0.4323419 & -2.0525366 \\ \text { C C6 } & -0.7360516 & -0.3851233 & -2.3978107 \\ \text { H H8 } & 1.3386951 & -0.6701132 & -2.7860423 \\ \text { C C8 } & 1.0303050 & -1.8232864 & 1.7058872 \\ \text { C C7 } & 0.1969418 & 2.2106470 & 1.1186024 \\ \text { H H2 } & 0.4331543 & 2.7170717 & 2.0577507 \\ \text { H H3 } & -0.7874880 & 2.5283995 & 0.7546868 \\ \text { H H4 } & 0.9582556 & 2.4414822 & 0.3645209 \\ \text { H H5 } & 0.6609934 & -2.6100888 & 2.3696133 \\ \text { H H6 } & 1.6711486 & -1.1300506 & 2.2618650 \\ \text { H H7 } & 1.5969461 & -2.2645649 & 0.8777166\end{array}$

Point Group $=\mathrm{C} 1$ Order $=1$ Nsymop $=1$

This system has 57 degrees of freedom

Coordinates read from pre-optimization

Cartesian Coordinates (Angstroms)

\begin{tabular}{|c|c|c|c|}
\hline \multicolumn{2}{|c|}{ Atom Label } & \multicolumn{2}{|l|}{ Y } \\
\hline H H17 & -4.3297909 & 0.6475361 & 1.1421814 \\
\hline H H1 & -2.0581129 & -1.1028688 & -6.4557701 \\
\hline $\mathrm{C} \mathrm{C} 2$ & -3.2511300 & -0.1114453 & -2.6491577 \\
\hline $\mathrm{Br} \mathrm{Br} 1$ & -6.5408949 & 0.2630861 & -3.8883436 \\
\hline C C1 & -2.8122014 & 0.2035915 & -0.1978005 \\
\hline C C3 & -0.1602265 & -0.1449658 & 0.8604683 \\
\hline $\mathrm{O} \mathrm{O} 2$ & -0.3184899 & -2.3423479 & 2.3782715 \\
\hline $\mathrm{OO} \mathrm{O} 3$ & 0.3837569 & 1.6160637 & 2.8004732 \\
\hline C C4 & 1.8944470 & -0.2497363 & -1.2635325 \\
\hline $\mathrm{O} \mathrm{O} 1$ & 4.1129002 & 0.1212066 & -0.8039614 \\
\hline $\mathrm{C} \mathrm{C5}$ & 1.0555994 & -0.8170082 & -3.8787336 \\
\hline C C6 & -1.3909364 & -0.7277779 & -4.5312073 \\
\hline H H8 & 2.5297682 & -1.2663309 & -5.2648591 \\
\hline C C8 & 1.9469951 & -3.4455134 & 3.2236608 \\
\hline C C7 & 0.3721663 & 4.1775190 & 2.1138530 \\
\hline H H2 & 0.8185433 & 5.1345235 & 3.8885869 \\
\hline H H3 & -1.4881373 & 4.7779844 & 1.4261520 \\
\hline H H4 & 1.8108414 & 4.6137346 & 0.6888449 \\
\hline H H5 & 1.2490970 & -4.9323549 & 4.4779219 \\
\hline
\end{tabular}




$\begin{array}{llll}\text { H H6 } & 3.1580144 & -2.1354870 & 4.2743071 \\ \text { H H7 } & 3.0177919 & -4.2794092 & 1.6586446\end{array}$

Initial Hessian option

Hessian from PM3D calculation used.

Max. Max. Neg.

Cycle Energy Grad. Dist. Eigen

$\begin{array}{llll}1 & -3089.7764908 & 0.03590 & 0.01246\end{array}$

$\begin{array}{llll}2 & -3089.7905566 & 0.01839 & 0.02377\end{array}$

$\begin{array}{llll}3 & -3089.7958166 & 0.00757 & 0.02271\end{array}$

$\begin{array}{llll}4 & -3089.7970004 & 0.00771 & 0.04167\end{array}$

$\begin{array}{lllll}5 & -3089.7973553 & 0.00478 & 0.04023\end{array}$

$\begin{array}{lllll}6 & -3089.7977528 & 0.00311 & 0.04264\end{array}$

$\begin{array}{llll}7 & -3089.7980092 & 0.00533 & 0.03665\end{array}$

$\begin{array}{lllll}8 & -3089.7981756 & 0.00481 & 0.02606\end{array}$

$\begin{array}{llll}9 & -3089.7983838 & 0.00215 & 0.03204\end{array}$

$\begin{array}{lllll}10 & -3089.7984699 & 0.00595 & 0.03112\end{array}$

$\begin{array}{lllll}11 & -3089.7984717 & 0.00456 & 0.01128\end{array}$

$\begin{array}{lllll}12 & -3089.7986165 & 0.00236 & 0.01467\end{array}$

$\begin{array}{lllll}13 & -3089.7986195 & 0.00409 & 0.00841\end{array}$

$\begin{array}{llll}14 & -3089.7986779 & 0.00262 & 0.00613\end{array}$

$\begin{array}{llll}15 & -3089.7986999 & 0.00253 & 0.00211\end{array}$

$\begin{array}{lllll}16 & -3089.7987482 & 0.00068 & 0.00517\end{array}$

$\begin{array}{lllll}17 & -3089.7987665 & 0.00183 & 0.00345\end{array}$

$\begin{array}{lllll}18 & -3089.7987959 & 0.00116 & 0.00508\end{array}$

$\begin{array}{lllll}19 & -3089.7988211 & 0.00134 & 0.01040\end{array}$

$\begin{array}{lllll}20 & -3089.7988541 & 0.00107 & 0.00907\end{array}$

$\begin{array}{llll}21 & -3089.7988870 & 0.00156 & 0.01459\end{array}$

$\begin{array}{lllll}22 & -3089.7989127 & 0.00202 & 0.01764\end{array}$

$\begin{array}{lllll}23 & -3089.7989837 & 0.00204 & 0.02786\end{array}$

$\begin{array}{lllll}24 & -3089.7990386 & 0.00174 & 0.01892\end{array}$

$\begin{array}{lllll}25 & -3089.7990180 & 0.00366 & 0.02127\end{array}$

$\begin{array}{llll}26 & -3089.7989766 & 0.00452 & 0.02485\end{array}$

$\begin{array}{lllll}27 & -3089.7990505 & 0.00313 & 0.00217\end{array}$

$\begin{array}{lllll}28 & -3089.7990627 & 0.00252 & 0.00209\end{array}$

$\begin{array}{lllll}29 & -3089.7990870 & 0.00097 & 0.00042\end{array}$

$\begin{array}{lllll}30 & -3089.7990914 & 0.00135 & 0.00008\end{array}$

$\begin{array}{lllll}31 & -3089.7990991 & 0.00060 & 0.00020\end{array}$

$\begin{array}{lllll}32 & -3089.7991034 & 0.00046 & 0.00029\end{array}$

$\begin{array}{llll}33 & -3089.7991086 & 0.00048 & 0.00061\end{array}$

$\begin{array}{lllll}34 & -3089.7991160 & 0.00041 & 0.00108\end{array}$

$\begin{array}{lllll}35 & -3089.7991247 & 0.00034 & 0.00197\end{array}$

$\begin{array}{lllll}36 & -3089.7991317 & 0.00033 & 0.00283\end{array}$

$\begin{array}{lllll}37 & -3089.7991340 & 0.00035 & 0.00008\end{array}$

$\begin{array}{lllll}38 & -3089.7991350 & 0.00028 & 0.00010\end{array}$

\begin{tabular}{cccc} 
& \multicolumn{2}{c}{ Cartesian Coordinates (Angstroms) } \\
Atom Label & $\mathrm{X}$ & $\mathrm{Y}$ & $\mathrm{Z}$
\end{tabular} 


$\begin{array}{lrrr}\text { H H17 } & -2.2518627 & 0.1589970 & 0.6621327 \\ \text { H H1 } & -1.1525413 & -0.3986197 & -3.4106972 \\ \text { C C2 } & -1.8299959 & -0.0541680 & -1.3758206 \\ \text { Br Br1 } & -3.6459602 & 0.1072062 & -1.9877373 \\ \text { C C1 } & -1.5202194 & 0.0227254 & -0.1046484 \\ \text { C C3 } & -0.1084528 & -0.1598953 & 0.3878590 \\ \text { O O2 } & -0.0923780 & -1.4588197 & 0.9577091 \\ \text { O O3 } & 0.1920362 & 0.7665406 & 1.4083617 \\ \text { C C4 } & 0.9477710 & -0.1435105 & -0.7258862 \\ \text { O O1 } & 2.1262351 & -0.0157184 & -0.4594073 \\ \text { C C5 } & 0.4751134 & -0.3408672 & -2.1025255 \\ \text { C C6 } & -0.8124921 & -0.2849352 & -2.4027514 \\ \text { H H8 } & 1.2259635 & -0.4882215 & -2.8502817 \\ \text { C C8 } & 1.0022828 & -1.7647601 & 1.8692206 \\ \text { C C7 } & 0.3851189 & 2.1465071 & 1.0246375 \\ \text { H H2 } & 0.4780674 & 2.6981617 & 1.9457296 \\ \text { H H3 } & -0.4654805 & 2.5176750 & 0.4649067 \\ \text { H H4 } & 1.2856415 & 2.2589888 & 0.4401052 \\ \text { H H5 } & 0.8831921 & -2.8114946 & 2.0975328 \\ \text { H H6 } & 0.9199140 & -1.1673305 & 2.7611625 \\ \text { H H7 } & 1.9580478 & -1.5884612 & 1.4003982 \\ \text { E(HF) }= & -3089.7991350 & & \\ \end{array}$

Compound $\mathbf{4 e}$

Run type: Geometry optimization

Model: RHF/3-21G

Number of shells: 57

$30 \mathrm{~S}$ shells

26 SP shells

$16 \mathrm{D}$ shells

Number of basis functions: 140

Number of electrons: 116

Use of molecular symmetry disabled

Molecular charge: 0

Spin multiplicity: 1

\begin{tabular}{lccc}
\multicolumn{5}{c}{ Cartesian Coordinates (Angstroms) } \\
Atom Label & X & Y & Z \\
----------- \\
H H17 & -0.3407448 & -2.2923151 & 0.6394466 \\
Br Br1 & 0.6640152 & -1.3072713 & -4.0767923 \\
C C2 & 0.0333922 & -1.7940082 & -1.3947730 \\
H H9 & -0.0721326 & -2.8088463 & -1.7948836 \\
C C1 & -0.1145061 & -1.5096149 & -0.0954205 \\
C C3 & 0.0726741 & -0.1059667 & 0.4639865 \\
O O2 & 1.2386441 & -0.1957913 & 1.2628739 \\
O O3 & -0.8561174 & 0.1778847 & 1.4963265
\end{tabular}




$\begin{array}{lccc}\text { C C4 } & 0.1258318 & 0.9987440 & -0.6422400 \\ \text { O O1 } & -0.0558485 & 2.1721496 & -0.3899384 \\ \text { C C5 } & 0.4071207 & 0.5542301 & -2.0296126 \\ \text { C C6 } & 0.3430163 & -0.7422260 & -2.3236042 \\ \text { H H8 } & 0.6396289 & 1.3112091 & -2.7879843 \\ \text { C C8 } & 1.8211566 & 0.9977791 & 1.7254827 \\ \text { C C7 } & -2.2122657 & 0.1753468 & 1.1362639 \\ \text { H H2 } & -2.7166458 & 0.4029385 & 2.0785438 \\ \text { H H3 } & -2.5316202 & -0.8051240 & 0.7633905 \\ \text { H H4 } & -2.4442237 & 0.9438302 & 0.3900684 \\ \text { H H5 } & 2.6062853 & 0.6207793 & 2.3867553 \\ \text { H H6 } & 1.1274884 & 1.6327806 & 2.2872252 \\ \text { H H7 } & 2.2648512 & 1.5734916 & 0.9048856\end{array}$

Point Group $=\mathrm{C} 1$ Order $=1$ Nsymop $=1$

This system has 57 degrees of freedom

Coordinates read from pre-optimization

Cartesian Coordinates (Angstroms)

$\begin{array}{lccc}\text { Atom Label } & \text { X } & \text { Y } & \text { Z } \\ \text {-- ------ } & --------------------- & \\ \text { H H17 } & -0.6439147 & -4.3318494 & 1.2083795 \\ \text { Br Br1 } & 1.2548073 & -2.4703858 & -7.7040239 \\ \text { C C2 } & 0.0631021 & -3.3901855 & -2.6357401 \\ \text { H H9 } & -0.1363109 & -5.3079523 & -3.3918397 \\ \text { C C1 } & -0.2163852 & -2.8527598 & -0.1803187 \\ \text { C C3 } & 0.1373342 & -0.2002482 & 0.8768077 \\ \text { O O2 } & 2.3406991 & -0.3699920 & 2.3864867 \\ \text { O O3 } & -1.6178280 & 0.3361535 & 2.8276484 \\ \text { C C4 } & 0.2377878 & 1.8873534 & -1.2136582 \\ \text { O O1 } & -0.1055385 & 4.1047694 & -0.7368771 \\ \text { C C5 } & 0.7693469 & 1.0473435 & -3.8354135 \\ \text { C C6 } & 0.6482072 & -1.4026045 & -4.3909774 \\ \text { H H8 } & 1.2087240 & 2.4778270 & -5.2685288 \\ \text { C C8 } & 3.4414886 & 1.8855300 & 3.2606910 \\ \text { C C7 } & -4.1805780 & 0.3313575 & 2.1472284 \\ \text { H H2 } & -5.1337186 & 0.7614438 & 3.9278800 \\ \text { H H3 } & -4.7840707 & -1.5214645 & 1.4425996 \\ \text { H H4 } & -4.6189152 & 1.7835813 & 0.7371228 \\ \text { H H5 } & 4.9251674 & 1.1731034 & 4.5103156 \\ \text { H H6 } & 2.1306452 & 3.0855094 & 4.3222310 \\ \text { H H7 } & 4.2799501 & 2.9734694 & 1.7099867\end{array}$

Initial Hessian option

Hessian from PM3D calculation used.

Max. Max. Neg.

Cycle Energy Grad. Dist. Eigen

$\begin{array}{llll}1 & -3089.7780207 & 0.03623 & 0.01393\end{array}$

$\begin{array}{llll}2 & -3089.7916533 & 0.01829 & 0.02741\end{array}$ 


$\begin{array}{rrrr}3 & -3089.7965017 & 0.00653 & 0.02753 \\ 4 & -3089.7973955 & 0.00767 & 0.03751 \\ 5 & -3089.7976376 & 0.00587 & 0.04179 \\ 6 & -3089.7980599 & 0.00267 & 0.05005 \\ 7 & -3089.7982958 & 0.00477 & 0.03180 \\ 8 & -3089.7984541 & 0.00466 & 0.01914 \\ 9 & -3089.7986443 & 0.00215 & 0.03085 \\ 10 & -3089.7987232 & 0.00522 & 0.03073 \\ 11 & -3089.7988077 & 0.00382 & 0.00640 \\ 12 & -3089.7989031 & 0.00200 & 0.00540 \\ 13 & -3089.7989393 & 0.00233 & 0.00806 \\ 14 & -3089.7989600 & 0.00247 & 0.00159 \\ 15 & -3089.7989710 & 0.00206 & 0.00351 \\ 16 & -3089.7990193 & 0.00093 & 0.00715 \\ 17 & -3089.7990183 & 0.00188 & 0.00335 \\ 18 & -3089.7990349 & 0.00205 & 0.00133 \\ 19 & -3089.7990332 & 0.00203 & 0.00148 \\ 20 & -3089.7990641 & 0.00083 & 0.00120 \\ 21 & -3089.7990735 & 0.00132 & 0.00162 \\ 22 & -3089.7990930 & 0.00115 & 0.00171 \\ 23 & -3089.7991195 & 0.00104 & 0.00320 \\ 24 & -3089.7991499 & 0.00129 & 0.00543 \\ 25 & -3089.7991873 & 0.00095 & 0.00958 \\ 26 & -3089.7992211 & 0.00166 & 0.02017 \\ 27 & -3089.7992380 & 0.00253 & 0.00849 \\ 28 & -3089.7992457 & 0.00245 & 0.00856 \\ 29 & -3089.7992723 & 0.00108 & 0.00106 \\ 30 & -3089.7992844 & 0.00105 & 0.00267 \\ 31 & -3089.7992991 & 0.00104 & 0.00595 \\ 32 & -3089.7993161 & 0.00129 & 0.00516 \\ 33 & -3089.7993312 & 0.00098 & 0.00460 \\ 34 & -3089.7993394 & 0.00053 & 0.00420 \\ 35 & -3089.7993388 & 0.00109 & 0.00079 \\ 36 & -3089.7993336 & 0.00121 & 0.00134 \\ 37 & -3089.7993420 & 0.00024 & 0.00026\end{array}$

Cartesian Coordinates (Angstroms)

$\begin{array}{lccc}\text { Atom Label } & \text { X } & \text { Y } & \text { Z } \\ \text {-- ----- } & --------1 & \\ \text { H H17 } & -0.1447093 & -2.2791139 & 0.6841546 \\ \text { Br Br1 } & 0.4373057 & -1.4406548 & -4.1859698 \\ \text { C C2 } & 0.0480151 & -1.8610763 & -1.3589004 \\ \text { H H9 } & -0.0346333 & -2.8728801 & -1.6938745 \\ \text { C C1 } & -0.0192371 & -1.5430748 & -0.0824168 \\ \text { C C3 } & 0.1590485 & -0.1372422 & 0.4182092 \\ \text { O O2 } & 1.4544851 & -0.1195568 & 0.9962433 \\ \text { O O3 } & -0.7738491 & 0.1679814 & 1.4334748 \\ \text { C C4 } & 0.1451188 & 0.9183160 & -0.6963756\end{array}$




$\begin{array}{lrrr}\text { O O1 } & 0.0363534 & 2.0991139 & -0.4324424 \\ \text { C C5 } & 0.3188088 & 0.4534674 & -2.0802185 \\ \text { C C6 } & 0.2603520 & -0.8295982 & -2.3760676 \\ \text { H H8 } & 0.4544693 & 1.2067160 & -2.8254700 \\ \text { C C8 } & 1.7568707 & 0.9780248 & 1.9048865 \\ \text { C C7 } & -2.1511628 & 0.3570824 & 1.0399377 \\ \text { H H2 } & -2.7087593 & 0.4598764 & 1.9564602 \\ \text { H H3 } & -2.5195692 & -0.4987939 & 0.4867148 \\ \text { H H4 } & -2.2608886 & 1.2520257 & 0.4455982 \\ \text { H H5 } & 2.7972948 & 0.8466100 & 2.1554363 \\ \text { H H6 } & 1.1407914 & 0.9109166 & 2.7851540 \\ \text { H H7 } & 1.6038943 & 1.9318602 & 1.4254660 \\ & & & \\ \text { E(HF })= & -3089.7993420 \text { a.u. } & \end{array}$

Compound $\mathbf{4 f}$

Run type: Geometry optimization

Model: RHF/3-21G

Number of shells: 63

$37 \mathrm{~S}$ shells

26 SP shells

Number of basis functions: 141

Number of electrons: 98

Use of molecular symmetry disabled

Molecular charge: 0

Spin multiplicity: 1

\begin{tabular}{lrrrr}
\multicolumn{5}{c}{ Cartesian Coordinates (Angstroms) } \\
Atom Label & X & Y & Z \\
-- & & & & \\
O O4 & 2.1364063 & -0.5133590 & 0.3354229 \\
H H1 & 0.5650166 & 0.4959201 & -3.7602201 \\
C C2 & 1.3296412 & 0.0225554 & -1.7770293 \\
H H9 & 2.3528808 & -0.0551903 & -2.1651455 \\
C C1 & 1.0716357 & -0.1329124 & -0.4608752 \\
C C3 & -0.3437698 & 0.0015893 & 0.1225513 \\
O O2 & -0.3611863 & 1.2455845 & 0.8010753 \\
O O3 & -0.5785631 & -0.8332677 & 1.2396513 \\
C C4 & -1.4305704 & -0.1172475 & -0.9964702 \\
O O1 & -2.5724515 & -0.4453203 & -0.7390573 \\
C C5 & -1.0221941 & 0.2081932 & -2.3779830 \\
C C6 & 0.2698945 & 0.2682692 & -2.7294364 \\
H H8 & -1.8233175 & 0.3698877 & -3.1092329 \\
C C8 & -1.6097060 & 1.7841478 & 1.1625131 \\
C C7 & -0.5609096 & -2.2202211 & 1.0180134 \\
H H2 & -0.7489726 & -2.6240894 & 2.0161201 \\
H H3 & 0.4115684 & -2.5652370 & 0.6483670 \\
H H4 & -1.3507069 & -2.5407410 & 0.3290816
\end{tabular}




\begin{tabular}{lccc} 
H H5 & -1.3159105 & 2.6492141 & 1.7631528 \\
H H6 & -2.2227563 & 1.1003718 & 1.7595169 \\
H H7 & -2.1737923 & 2.1112990 & 0.2816758 \\
C C9 & 2.4170966 & 0.3095039 & 1.4470463 \\
H H10 & 2.6123713 & 1.3443473 & 1.1475575 \\
H H11 & 3.3271232 & -0.1480201 & 1.8444284 \\
H H12 & 1.6211724 & 0.2847231 & 2.1992759 \\
\multicolumn{5}{c}{} \\
Point Group $=$ C1 Order & $=1$ Nsymop & 1 \\
This system has 69 degrees of freedom \\
Coordinates read from pre-optimization \\
\multicolumn{5}{c}{} \\
\multicolumn{5}{c}{ Cartesian Coordinates (Angstroms) } \\
Atom Label & X & Y \\
-- ------- --------------------------------- \\
O O4 & 4.0372244 & -0.9701083 & 0.6338576 \\
H H1 & 1.0677270 & 0.9371535 & -7.1057890 \\
C C2 & 2.5126587 & 0.0426236 & -3.3581000 \\
H H9 & 4.4463021 & -0.1042946 & -4.0915336 \\
C C1 & 2.0250987 & -0.2511681 & -0.8709282 \\
C C3 & -0.6496311 & 0.0030034 & 0.2315884 \\
O O2 & -0.6825435 & 2.3538146 & 1.5138135 \\
O O3 & -1.0933263 & -1.5746484 & 2.3426024 \\
C C4 & -2.7033874 & -0.2215658 & -1.8830566 \\
O O1 & -4.8612307 & -0.8415338 & -1.3966164 \\
C C5 & -1.9316677 & 0.3934283 & -4.4937383 \\
C C6 & 0.5100268 & 0.5069556 & -5.1578893 \\
H H8 & -3.4455721 & 0.6989867 & -5.8756010 \\
C C8 & -3.0419047 & 3.3715520 & 2.1968322 \\
C C7 & -1.0599660 & -4.1956115 & 1.9237672 \\
H H2 & -1.4153536 & -4.9588122 & 3.8099164 \\
H H3 & 0.7777519 & -4.8475973 & 1.2252366 \\
H H4 & -2.5524672 & -4.8013066 & 0.6218744 \\
H H5 & -2.4867115 & 5.0062910 & 3.3318773 \\
H H6 & -4.2004023 & 2.0794022 & 3.3250064 \\
H H7 & -4.1078738 & 3.9897784 & 0.5322904 \\
C C9 & 4.5676525 & 0.5848779 & 2.7345223 \\
H H10 & 4.9366683 & 2.5404492 & 2.1685702 \\
H H11 & 6.2873541 & -0.2797176 & 3.4854660 \\
H H12 & 3.0635731 & 0.5380489 & 4.1560307
\end{tabular}

Initial Hessian option

Hessian from PM3D calculation used.

Max. Max. Neg.

Cycle Energy Grad. Dist. Eigen

$\begin{array}{llll}1 & -643.5526843 & 0.03880 & 0.01258\end{array}$

$\begin{array}{lllll}2 & -643.5679576 & 0.01859 & 0.02807\end{array}$

$\begin{array}{lllll}3 & -643.5736169 & 0.00842 & 0.03079\end{array}$

$\begin{array}{llll}4 & -643.5752784 & 0.00458 & 0.04376\end{array}$ 


$\begin{array}{cccc}5 & -643.5760068 & 0.00275 & 0.04104 \\ 6 & -643.5764085 & 0.00519 & 0.04455 \\ 7 & -643.5767179 & 0.00440 & 0.04470 \\ 8 & -643.5769533 & 0.00330 & 0.03619 \\ 9 & -643.5771787 & 0.00219 & 0.03161 \\ 10 & -643.5773399 & 0.00176 & 0.04552 \\ 11 & -643.5774713 & 0.00235 & 0.02803 \\ 12 & -643.5775228 & 0.00434 & 0.01150 \\ 13 & -643.5775384 & 0.00816 & 0.02376 \\ 14 & -643.5775169 & 0.00690 & 0.03758 \\ 15 & -643.5774991 & 0.00509 & 0.01139 \\ 16 & -643.5776058 & 0.00286 & 0.00639 \\ 17 & -643.5776623 & 0.00229 & 0.00356 \\ 18 & -643.5776731 & 0.00124 & 0.00337 \\ 19 & -643.5776842 & 0.00116 & 0.00074 \\ 20 & -643.5776924 & 0.00074 & 0.00078 \\ 21 & -643.5777018 & 0.00134 & 0.00198 \\ 22 & -643.5777134 & 0.00129 & 0.00338 \\ 23 & -643.5777214 & 0.00098 & 0.00389 \\ 24 & -643.5777350 & 0.00113 & 0.00426 \\ 25 & -643.5777530 & 0.00062 & 0.00374 \\ 26 & -643.5777622 & 0.00059 & 0.00285 \\ 27 & -643.5777575 & 0.00188 & 0.00280 \\ 28 & -643.5777735 & 0.00132 & 0.00212 \\ 29 & -643.5777766 & 0.00095 & 0.00047 \\ 30 & -643.5777853 & 0.00044 & 0.00111 \\ 31 & -643.5777945 & 0.00060 & 0.00166 \\ 32 & -643.5778066 & 0.00083 & 0.00152 \\ 33 & -643.5778238 & 0.00108 & 0.00326 \\ 34 & -643.5778419 & 0.00112 & 0.00858 \\ 35 & -643.5778569 & 0.00107 & 0.00443 \\ 36 & -643.5778663 & 0.00126 & 0.00325 \\ 37 & -643.5778757 & 0.00065 & 0.00239 \\ 38 & -643.5778829 & 0.00090 & 0.00129 \\ 39 & -643.5778880 & 0.00066 & 0.00061 \\ 40 & -643.5778910 & 0.00042 & 0.00091 \\ 41 & -643.5778949 & 0.00043 & 0.00134 \\ 42 & -643.5778990 & 0.00044 & 0.00245 \\ 43 & -643.5779036 & 0.00028 & 0.00169 \\ 44 & -643.5779064 & 0.00030 & 0.00041 \\ 45 & -643.5779076 & 0.00021 & 0.00054 \\ 46 & -643.5779079 & 0.00051 & 0.00020 \\ 47 & -643.5779084 & 0.00019 & 0.00001\end{array}$

\begin{tabular}{lrrrr}
\multicolumn{5}{c}{ Cartesian Coordinates (Angstroms) } \\
Atom Label & X & Y & \multicolumn{1}{c}{ Z } \\
-- & & & & \\
\multicolumn{1}{c}{ O O4 } & 2.1191186 & -0.3599374 & 0.3457861 \\
H H1 & 0.5491190 & 0.1784890 & -3.8335930
\end{tabular}




$\begin{array}{lccc}\text { C C2 } & 1.3557609 & -0.0729118 & -1.8378914 \\ \text { H H9 } & 2.3615815 & -0.1614368 & -2.1886743 \\ \text { C C1 } & 1.1139599 & -0.1236310 & -0.5391522 \\ \text { C C3 } & -0.2851065 & 0.0714022 & 0.0046790 \\ \text { O O2 } & -0.2976577 & 1.4127399 & 0.4799923 \\ \text { O O3 } & -0.5381820 & -0.7711966 & 1.1057660 \\ \text { C C4 } & -1.3982054 & -0.0398795 & -1.0555089 \\ \text { O O1 } & -2.5491623 & -0.2010831 & -0.6971030 \\ \text { C C5 } & -1.0088816 & 0.1219213 & -2.4528243 \\ \text { C C6 } & 0.2724181 & 0.0915291 & -2.8013379 \\ \text { H H8 } & -1.7986169 & 0.2124615 & -3.1682127 \\ \text { C C8 } & -1.3976139 & 1.7918596 & 1.3566500 \\ \text { C C7 } & -0.5881438 & -2.1942450 & 0.8484964 \\ \text { H H2 } & -0.7516367 & -2.6605004 & 1.8061034 \\ \text { H H3 } & 0.3482301 & -2.5390798 & 0.4312145 \\ \text { H H4 } & -1.4058118 & -2.4333570 & 0.1850418 \\ \text { H H5 } & -1.2699234 & 2.8504991 & 1.5158926 \\ \text { H H6 } & -1.3328539 & 1.2556795 & 2.2884302 \\ \text { H H7 } & -2.3490844 & 1.5924363 & 0.8913711 \\ \text { C C9 } & 2.1878888 & 0.3723439 & 1.6071013 \\ \text { H H10 } & 2.1839431 & 1.4348245 & 1.4232138 \\ \text { H H11 } & 3.1208726 & 0.0661329 & 2.0496956 \\ \text { H H12 } & 1.3579879 & 0.1049402 & 2.2348633 \\ & & & \\ \text { E(HF) }= & -643.5779084 & a . u . & \end{array}$

\section{Additional References :}

(22) (a) Meyers, A. I.; Snyder, L. J. Org. Chem. 1993, 58, 36-42. (a) Van der Mey, M.; Hatzelmann, A.; Van Klink, G. P. M.; Van der Laan, I. J.; Sterk, G. J.; Thibaut, U.; Ulrich, W. R.; Timmerman, H. J. Med. Chem. 2001, 44, 2523-2535.

(23) Oberhauser, T. J. Org. Chem. 1997, 62, 4504-4506.

(24) Villemin, D.; Hammadi, M.; Hachemi, M. Synth. Comm. 2002, 32, 1501-1515.

(25) Corey, E. J.; Barcza, S.; Klotmann, G. J. Am. Chem. Soc. 1969, 91, 4782-4786. 
IFA $16.1 / \mathrm{CDCl} 3 / \mathrm{MA} 03-108 / \mathrm{AC} 250$
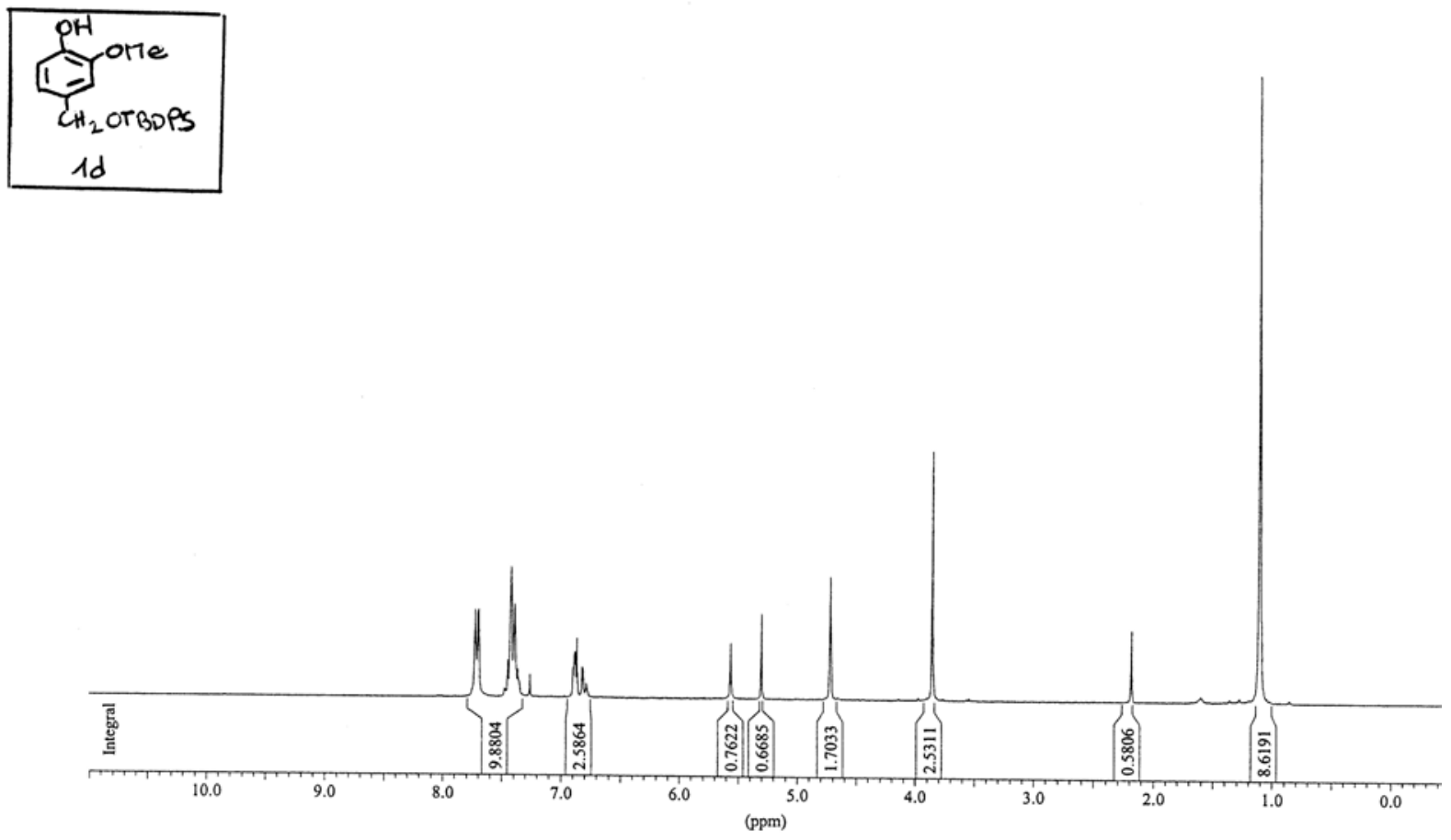
Peak List

File Name : D: \RMNDEA 1 \MAI200 1\MA03PR 1 1108001.1R

Peak Results saved in File: D: \RMNDEA 1\MAI200 1\MA03PR 1\108001. TXT Nucleus

$\mathrm{SF}$ : $1 \mathrm{H}$

: $250.132854 \mathrm{MHz}$

OFFSET

SW_p

: $15.7748 \mathrm{ppm}$

SI

: $5000.00 \mathrm{~Hz}$

: 16384

Peak Picking Parameter

Peak constant $\mathrm{PC}=$

1.00

Noise

3

Sens. level=

12

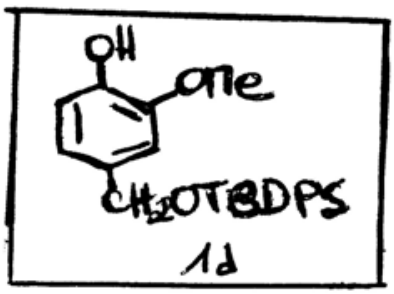

Peak Picking region

\begin{tabular}{llcc} 
Start $(\mathrm{ppm} / \mathrm{Hz})$ & End $(\mathrm{ppm} / \mathrm{Hz})$ & MI(\%) & MAXI(\%) \\
\hline $7.84 / 1961.2$ & $6.74 / 1685.0$ & 2.02 & 28.20 \\
$5.64 / 1410.1$ & $5.43 / 1358.5$ & 5.26 & 12.68 \\
$4.74 / 1184.6$ & $4.61 / 1154.3$ & 15.58 & 24.44 \\
$3.99 / 998.4$ & $3.73 / 934.0$ & 24.84 & 52.13 \\
$1.14 / 2285.8$ & $1.05 / 262.9$ & 62.65 & 114.48
\end{tabular}

Peak Picking results

\begin{tabular}{rrrrrr} 
Peak Nr. Data Point & Frequency & PPM & Intensity & \%Int. \\
\hline 1 & 6595 & 1933.15 & 7.7285 & 2306 & 10.5 \\
2 & 6600 & 1931.63 & 7.7224 & 3033 & 13.9 \\
3 & 6619 & 1925.83 & 7.6992 & 2908 & 13.3 \\
4 & 6625 & 1924.00 & 7.6919 & 3033 & 13.9 \\
5 & 6826 & 1862.66 & 7.4467 & 1269 & 5.8 \\
6 & 6851 & 1855.03 & 7.4162 & 4511 & 20.6 \\
7 & 6875 & 1847.70 & 7.3869 & 3223 & 14.7 \\
8 & 6894 & 1841.90 & 7.3637 & 966 & 4.4 \\
9 & 6903 & 1839.16 & 7.3527 & 685 & 3.1 \\
10 & 6979 & 1815.96 & 7.2600 & 783 & 3.6 \\
11 & 7276 & 1725.33 & 6.8976 & 1030 & 4.7 \\
12 & 7290 & 1721.05 & 6.8806 & 1578 & 7.2 \\
13 & 7303 & 1717.09 & 6.8647 & 2035 & 9.3 \\
14 & 7341 & 1705.49 & 6.8183 & 1052 & 4.8 \\
15 & 7368 & 1697.25 & 6.7854 & 493 & 2.3 \\
16 & 8371 & 1391.16 & 5.5617 & 1921 & 8.8 \\
17 & 9062 & 1180.28 & 4.7186 & 4275 & 19.5 \\
18 & 9771 & 963.91 & 3.8536 & 8652 & 39.5
\end{tabular}




$19 \quad 12023 \quad 276.66 \quad 1.1060 \quad 21888 \quad 100.0$

IFA $16.1 / \mathrm{CDC} 13 / \mathrm{MA} 03-108 / \mathrm{AC} 250$

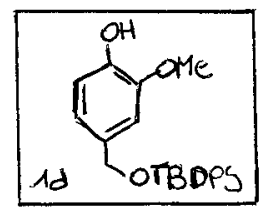

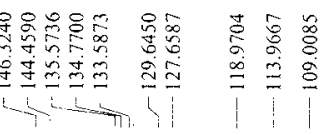

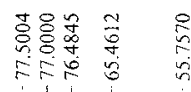

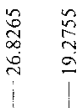

Ad OTBDPS

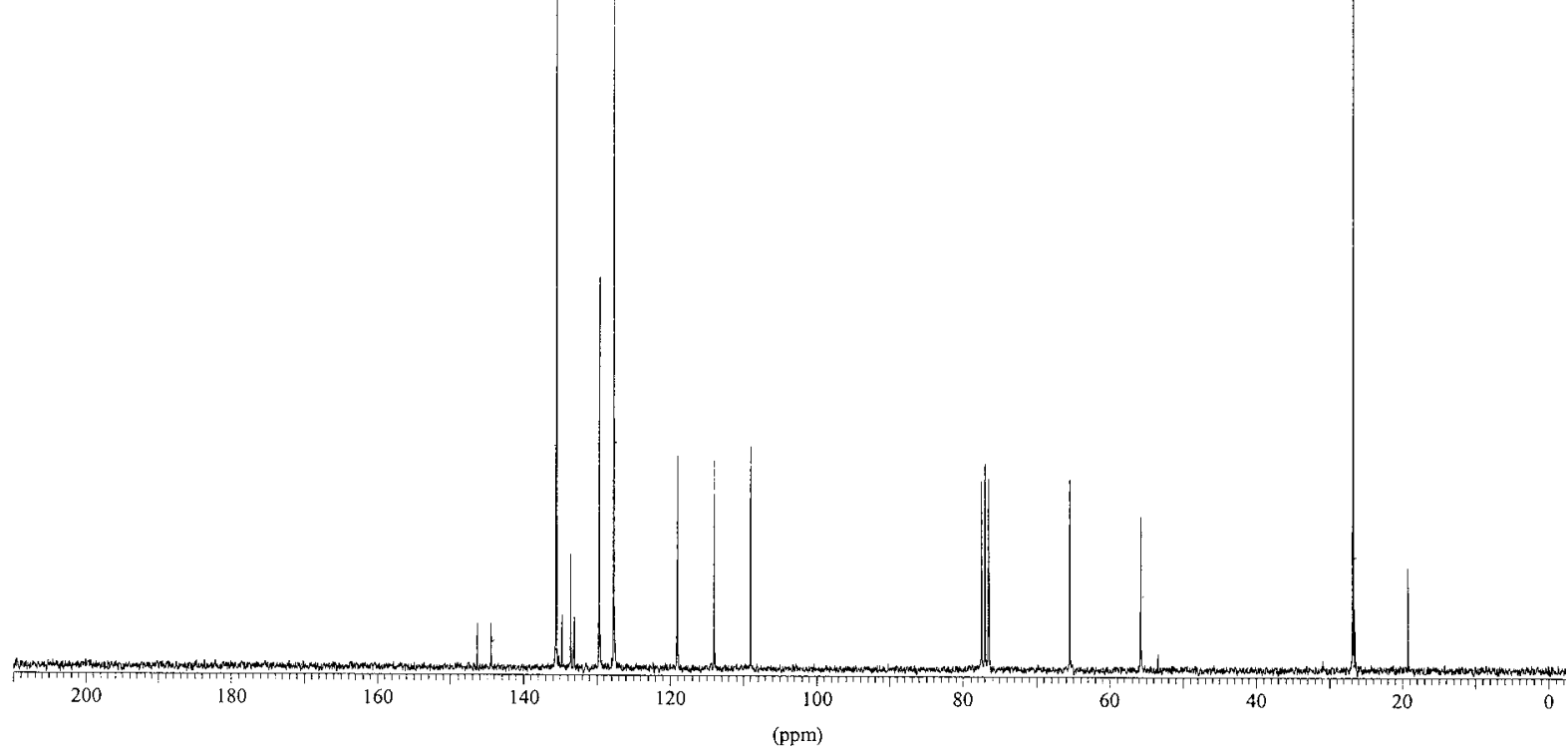


IFB 137.1/CDCI3/MI27-106/AC 250

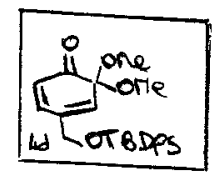

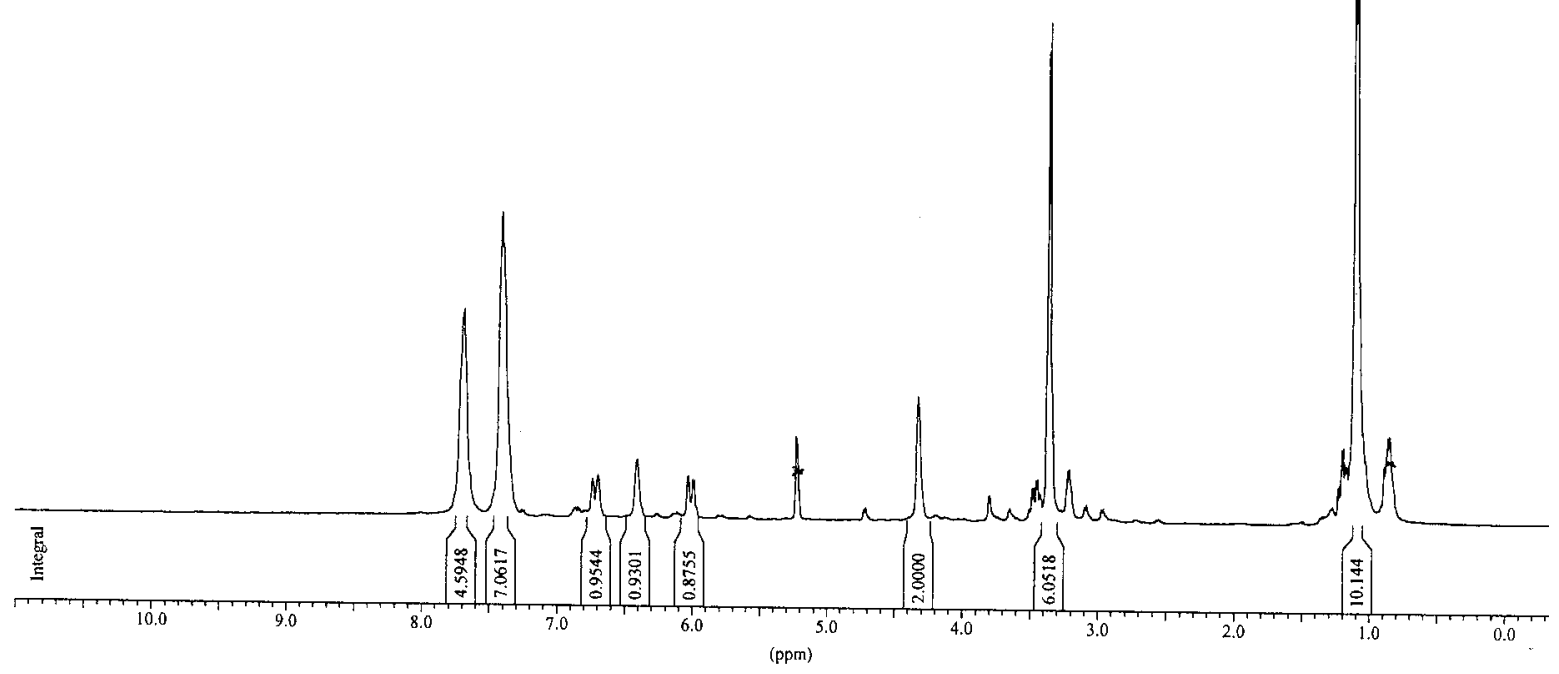




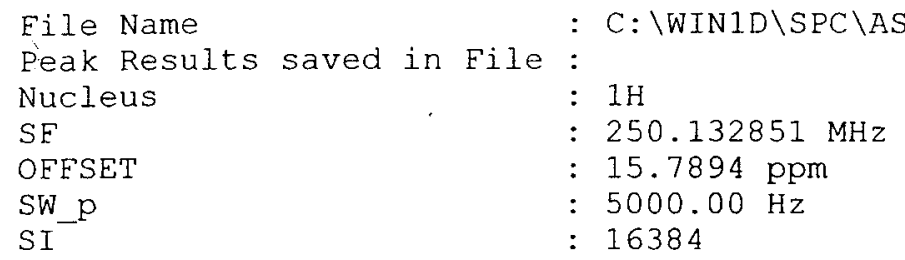

Peak Picking Parameter

$\begin{array}{lrr}\text { Peak constant } \mathrm{PC} & = & 1.00 \\ \text { Noise } & = & 1 \\ \text { Sens. level } & = & 4\end{array}$

Peak Picking region

\begin{tabular}{rrrr} 
Start $(\mathrm{ppm} / \mathrm{Hz})$ & End $(\mathrm{ppm} / \mathrm{Hz})$ & MI (\%) & MAXI (\%) \\
\hline $8.25 / 2063.2$ & $7.13 / 1784.2$ & 6.80 & 49.48 \\
$7.29 / 1824.5$ & $7.18 / 1795.2$ & 0.71 & 3.35 \\
$6.87 / 1718.3$ & $5.90 / 1476.0$ & 4.16 & 12.37 \\
$4.42 / 1105.2$ & $4.07 / 1018.8$ & 12.58 & 20.79 \\
$3.69 / 923.9$ & $3.02 / 755.2$ & 62.24 & 80.09 \\
$1.25 / 312.7$ & $0.80 / 200.7$ & 85.97 & 106.04 \\
\hline
\end{tabular}

Peak Picking results

\begin{tabular}{rrrrrr} 
Peak Nr. Data Point & Frequency & \multicolumn{1}{c}{ PPM } & Intensity & \%Int. \\
\hline 1 & 6617 & 1930.10 & 7.7163 & 665 & 19.7 \\
2 & 6634 & 1924.91 & 7.6956 & 920 & 27.3 \\
3 & 6641 & 1922.78 & 7.6870 & 952 & 28.2 \\
4 & 6866 & 1854.11 & 7.4125 & 1403 & 41.6 \\
5 & 6875 & 1851.36 & 7.4015 & 1262 & 37.4 \\
6 & 6991 & 1815.96 & 7.2600 & 26 & 0.8 \\
7 & 7173 & 1760.42 & 7.0379 & -2 & -0.1 \\
8 & 7419 & 1685.35 & 6.7378 & 170 & 5.0 \\
9 & 7451 & 1675.58 & 6.6988 & 192 & 5.7 \\
10 & 7684 & 1604.48 & 6.4145 & 254 & 7.5 \\
11 & 7689 & 1602.95 & 6.4084 & 265 & 7.8 \\
12 & 7992 & 1510.48 & 6.0387 & 181 & 5.4 \\
13 & 8001 & 1507.74 & 6.0277 & 189 & 5.6 \\
14 & 8025 & 1500.41 & 5.9985 & 171 & 5.1 \\
15 & 8034 & 1497.67 & 5.9875 & 169 & 5.0 \\
16 & 9397 & 1081.71 & 4.3245 & 566 & 16.8 \\
17 & 10176 & 843.98 & 3.3741 & 2175 & 64.4 \\
18 & 10188 & 840.32 & 3.3595 & 2310 & 68.4 \\
19 & 12024 & 280.01 & 1.1195 & 3061 & 90.7 \\
20 & 12037 & 276.05 & 1.1036 & 3376 & 100.0 \\
\hline
\end{tabular}



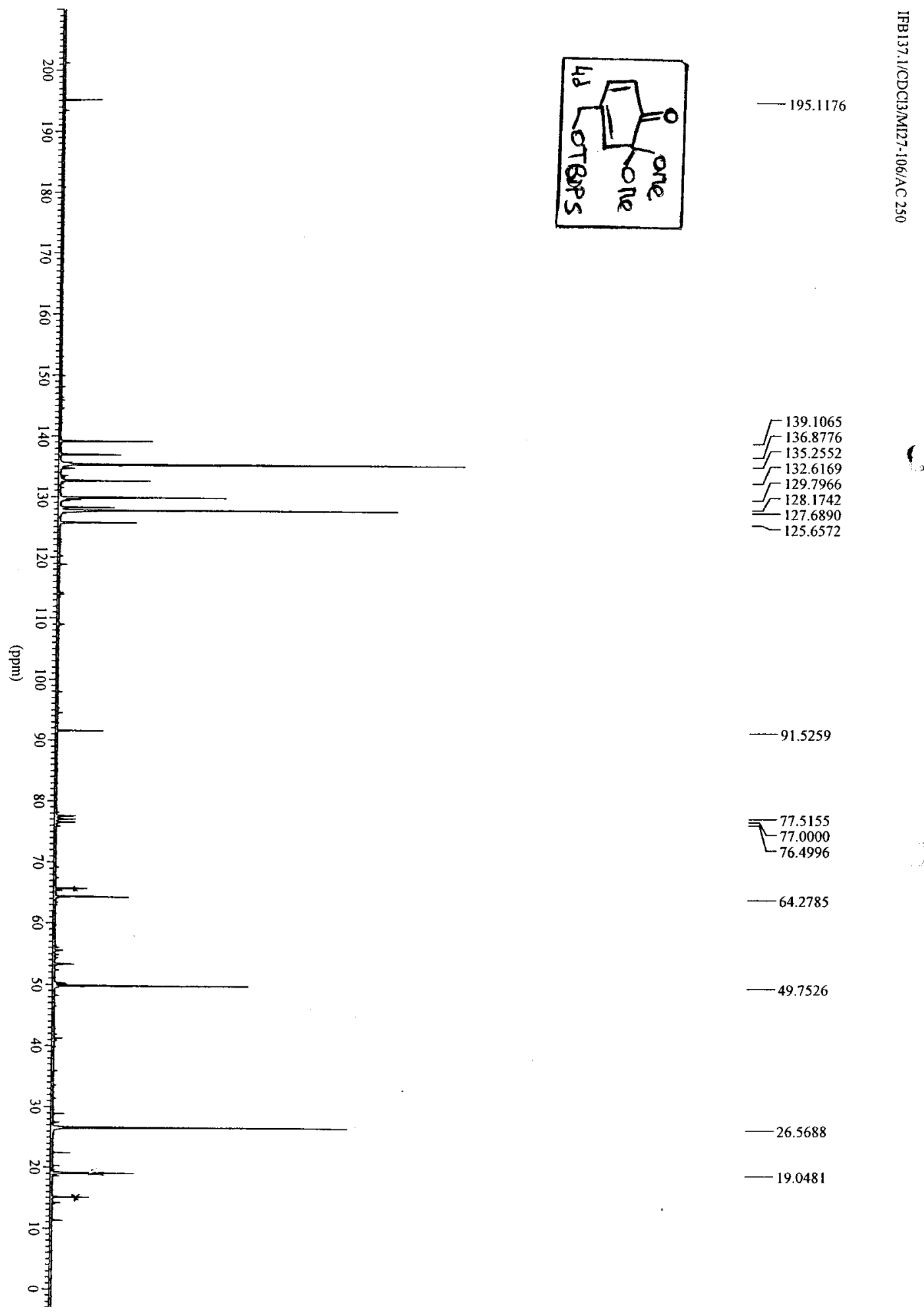

139.1065 136.8776
1352552 f 135.2552 132.6169
129.7966 $\tau^{128.1742}$

127.6890
-125.6502

$-125.6572$

$-91.5259$

77.5155

$-76.4996$

$-64.2785$

$-49.7526$

$-26.5688$

$-19.0481$ 
IFB!3CDCl3/08050326/DPX 300
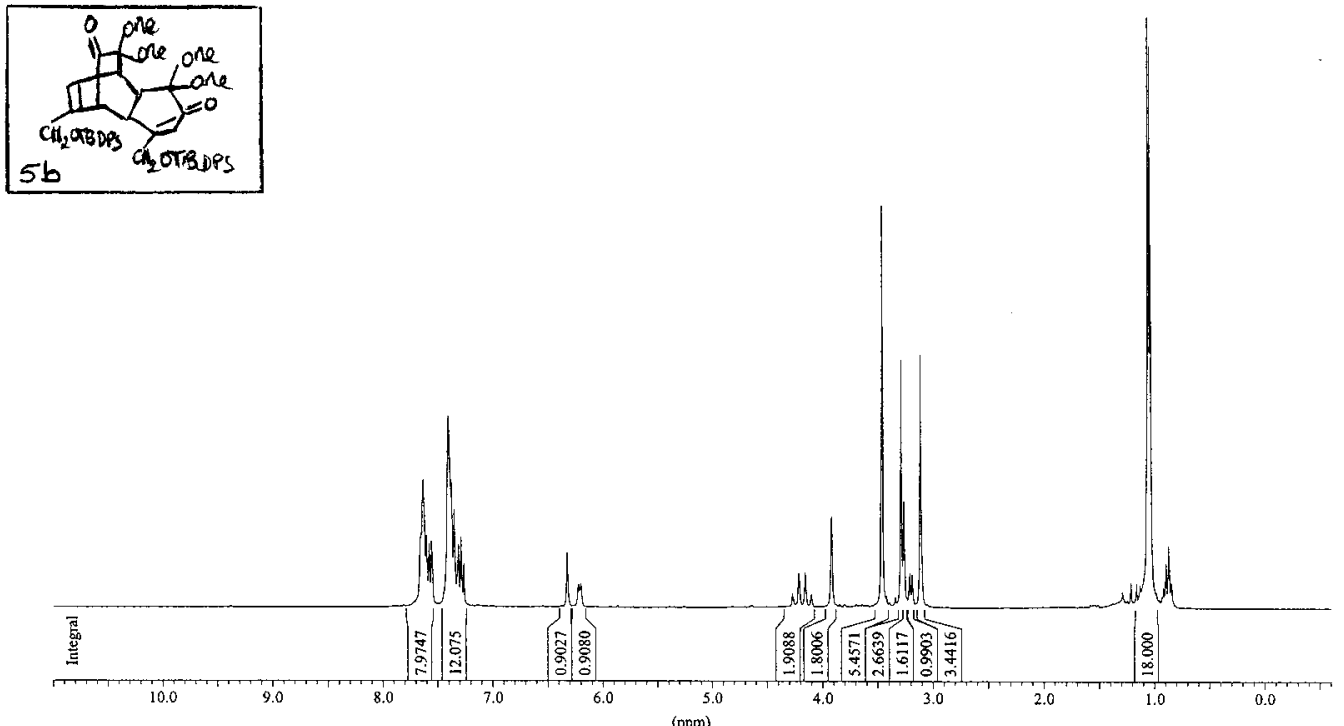
File Name $\quad$ : D: \DPX300 1 1 $08050326 \backslash 001001.1 R$ Peak Results saved in File : D: \DPX300 1\08050326\001001.TXT Nucleus : off

SF : $300.130006 \mathrm{MHz}$

OFFSET

SW_p

: $16.4402 \mathrm{ppm}$

: $6172.84 \mathrm{~Hz}$

SI

: 32768

Peak Picking Parameter

Peak constant $P C=1.00$

Noise $\quad=\quad 209$

Sens. level = 836

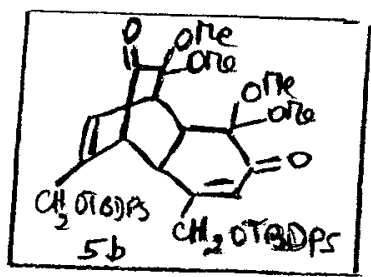

Peak Picking region

\begin{tabular}{|c|c|c|c|}
\hline Start $(\mathrm{ppm} / \mathrm{Hz})$ & End $(\mathrm{ppm} / \mathrm{Hz})$ & $M I(\%)$ & $\operatorname{MAXI}(\%)$ \\
\hline $7.69 / 2308.9$ & $7.17 / 2151.6$ & 5.10 & 34.51 \\
\hline $6.37 / 1913.1$ & $6.07 / 1820.5$ & 2.18 & 10.10 \\
\hline $4.36 / 1309.8$ & $4.07 / 1220.1$ & 1.82 & 23.35 \\
\hline $3.54 / 1061.5$ & $3.34 / 1001.8$ & 58.20 & 69.95 \\
\hline $3.31 / 994.4$ & $3.27 / 980.3$ & 36.97 & 44.16 \\
\hline $3.13 / 940.7$ & $3.09 / 926.0$ & 38.55 & 51.18 \\
\hline $4.50 / 1350.6$ & $3.73 / 1119.1$ & 9.80 & 31.40 \\
\hline $1.21 / 363.3$ & $0.91 / 274.0$ & 84.79 & 111.79 \\
\hline $3.23 / 970.5$ & $3.17 / 950.0$ & 3.92 & 9.47 \\
\hline
\end{tabular}

Peak Picking results

\begin{tabular}{rrrrrr}
\hline Peak Nr. Data Point & Frequency & PPM & Intensity & \%Int. \\
\hline 1 & 14001 & 2296.68 & 7.6523 & 52723 & 11.9 \\
2 & 14011 & 2294.80 & 7.6460 & 55183 & 12.4 \\
3 & 14042 & 2288.96 & 7.6266 & 94487 & 21.3 \\
4 & 14049 & 2287.64 & 7.6222 & 94713 & 21.3 \\
5 & 14084 & 2281.05 & 7.6002 & 53220 & 12.0 \\
6 & 14125 & 2273.32 & 7.5745 & 46966 & 10.6 \\
7 & 14160 & 2266.73 & 7.5525 & 48772 & 11.0 \\
8 & 14395 & 2222.46 & 7.4050 & 121294 & 27.3 \\
9 & 14405 & 2220.58 & 7.3987 & 143261 & 32.3 \\
10 & 14411 & 2219.45 & 7.3949 & 141438 & 31.9 \\
11 & 14427 & 2216.43 & 7.3849 & 111678 & 25.2 \\
12 & 14447 & 2212.66 & 7.3724 & 94841 & 21.4 \\
13 & 14490 & 2204.56 & 7.3454 & 72923 & 16.4 \\
14 & 14528 & 2197.40 & 7.3215 & 24470 & 5.5
\end{tabular}




$\begin{array}{rrrrrr}15 & 14550 & 2193.26 & 7.3077 & 46567 & 10.5 \\ 16 & 14589 & 2185.91 & 7.2832 & 52600 & 11.9 \\ 17 & 14626 & 2178.94 & 7.2600 & 32310 & 7.3 \\ 18 & 16124 & 1896.75 & 6.3198 & 40683 & 9.2 \\ 19 & 16288 & 1865.86 & 6.2168 & 17332 & 3.9 \\ 20 & 16323 & 1859.26 & 6.1949 & 17423 & 3.9 \\ 21 & 19397 & 1280.18 & 4.2654 & 10423 & 2.3 \\ 22 & 19484 & 1263.79 & 4.2108 & 25898 & 5.8 \\ 23 & 19575 & 1246.65 & 4.1537 & 26448 & 6.0 \\ 24 & 19663 & 1230.07 & 4.0985 & 10157 & 2.3 \\ 25 & 19957 & 1174.69 & 3.9139 & 67739 & 15.3 \\ 26 & 20687 & 1037.17 & 3.4557 & 302159 & 68.1 \\ 27 & 20958 & 986.12 & 3.2856 & 185558 & 41.8 \\ 28 & 20999 & 978.40 & 3.2599 & 79724 & 18.0 \\ 29 & 21084 & 962.38 & 3.2066 & 25504 & 5.7 \\ 30 & 21120 & 955.60 & 3.1840 & 24753 & 5.6 \\ 31 & 21240 & 933.00 & 3.1086 & 189955 & 42.8 \\ 32 & 24510 & 316.99 & 1.0562 & 443752 & 100.0 \\ 33 & 24540 & 311.34 & 1.0374 & 422303 & 95.2 \\ \ldots\end{array}$




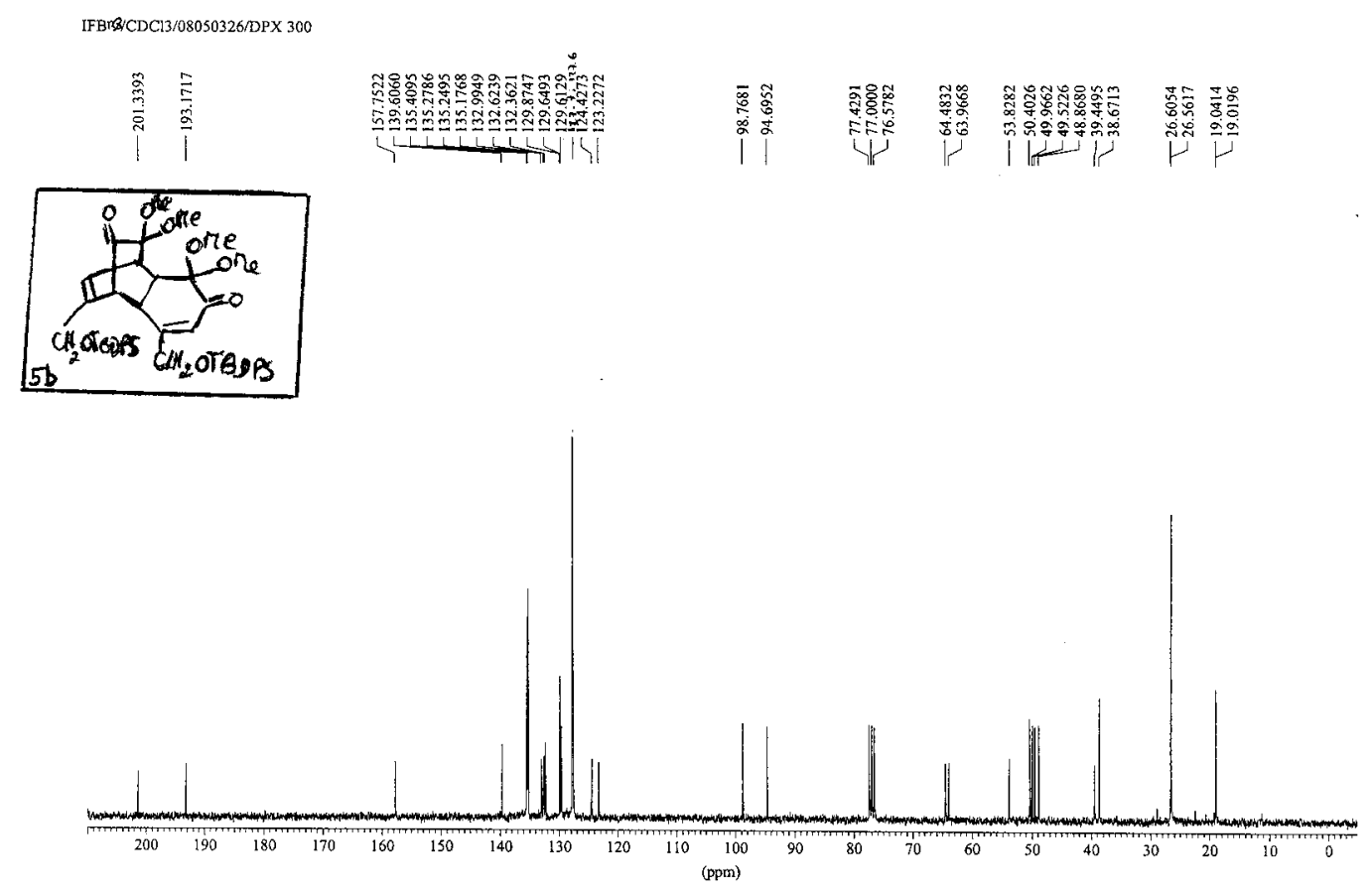

IFB131.3/CDC13/MI20-103/AC 250
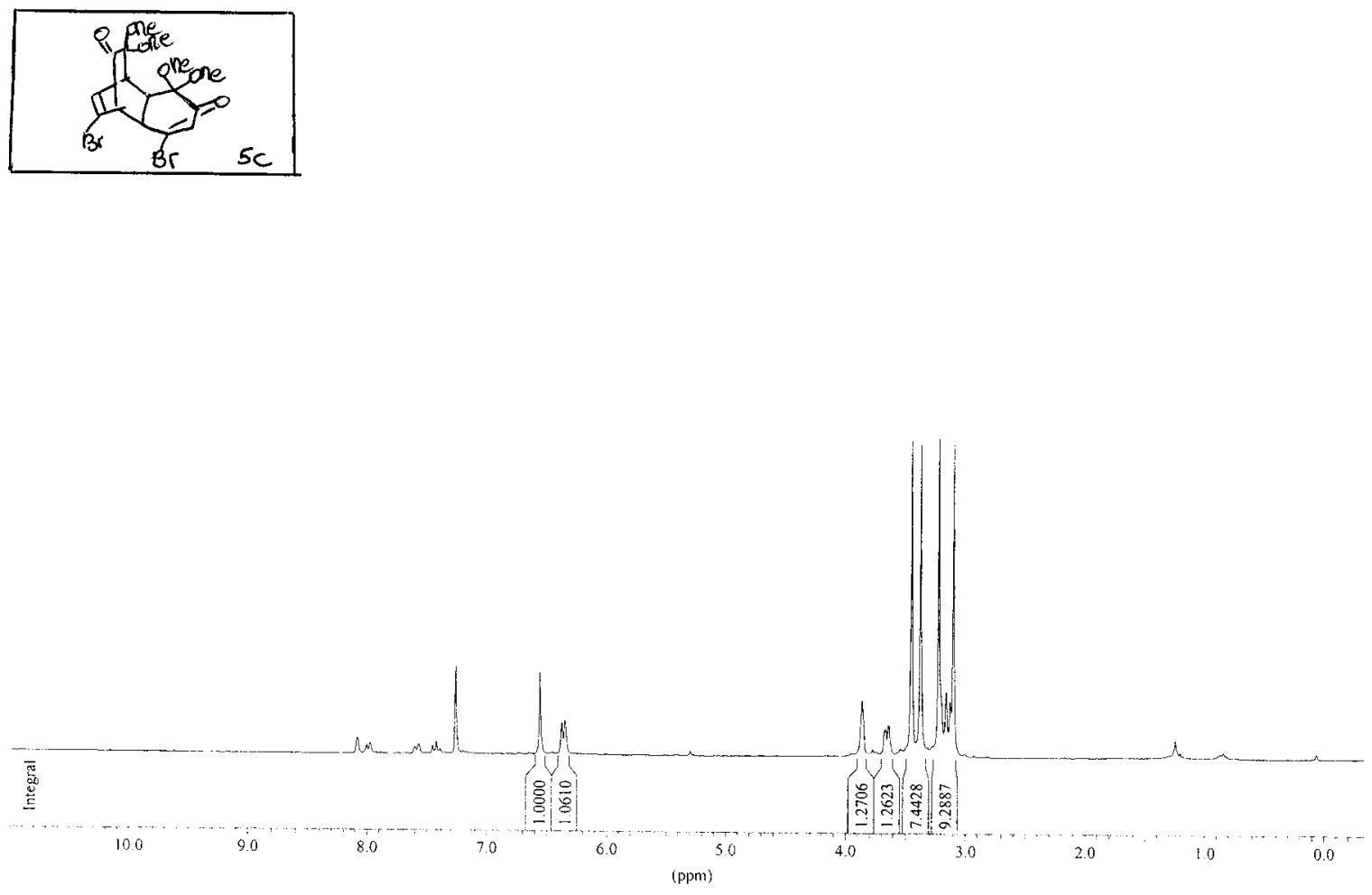
File Name

C: $\backslash W I N 1 D \backslash S P C \backslash A S P 3000 \backslash M I 200 F \backslash 103001.1 R$

Peak Results saved in File:

Nucleus

$S F$

$1 \mathrm{H}$

OFFSET

$250.132854 \mathrm{MHz}$

: $15.7760 \mathrm{ppm}$

SW_P

$5000.00 \mathrm{~Hz}$

$S I$

16384

Peak Picking Parameter

Peak constant $\mathrm{PC}=$

1.00

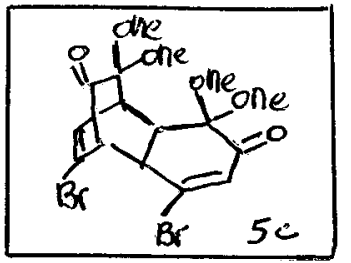

Noise

Sens. level

.00
1

Peak Picking region

\begin{tabular}{|c|c|c|c|}
\hline Start (ppm/Hz) & End (ppm/Hz) & $\operatorname{MI}(\%)$ & $\operatorname{MAXI}(\%)$ \\
\hline 2053.1 & 1746.7 & 21.30 & 39.13 \\
\hline 1657.6 & 1602.0 & 20.96 & 29.02 \\
\hline 1604.8 & 1562.1 & 8.74 & 11.30 \\
\hline 1004.2 & $3.80 \%$ & 15.85 & 31.98 \\
\hline 931.3 & $3.57 /$ & 8.23 & 12.80 \\
\hline $3.53 /$ & $2.81 /$ & 75.33 & 107.36 \\
\hline 793.6 & 3.091 & 16.07 & 23.91 \\
\hline
\end{tabular}

Peak Picking results

\begin{tabular}{rrrrrr} 
Peak Nr. Data Point & Frequency & PPM & Intensity & \% Int. \\
\hline 1 & 6980 & 1815.96 & 7.2600 & 1186 & 27.4 \\
2 & 7557 & 1639.88 & 6.5560 & 1106 & 25.6 \\
3 & 7704 & 1595.02 & 6.3767 & 417 & 9.7 \\
4 & 7710 & 1593.19 & 6.3694 & 430 & 10.0 \\
5 & 7727 & 1588.00 & 6.3486 & 452 & 10.5 \\
6 & 7733 & 1586.17 & 6.3413 & 442 & 10.2 \\
7 & 9767 & 965.44 & 3.8597 & 766 & 17.7 \\
8 & 9919 & 919.05 & 3.6743 & 370 & 8.6 \\
9 & 9928 & 916.31 & 3.6633 & 384 & 8.9 \\
10 & 9945 & 911.12 & 3.6425 & 442 & 10.2 \\
11 & 9954 & 908.37 & 3.6316 & 423 & 9.8 \\
12 & 10103 & 862.90 & 3.4498 & 4289 & 99.2 \\
13 & 10166 & 843.67 & 3.3729 & 4230 & 97.9 \\
14 & 10289 & 806.14 & 3.2228 & 4322 & 100.0 \\
15 & 10342 & 789.96 & 3.1582 & 904 & 20.9 \\
16 & 10367 & 782.33 & 3.1277 & 752 & 17.4 \\
17 & 10389 & 775.62 & 3.1008 & 4244 & 98.2 \\
\hline
\end{tabular}


[FB131.3/CDC13/M[20-103/AC 250

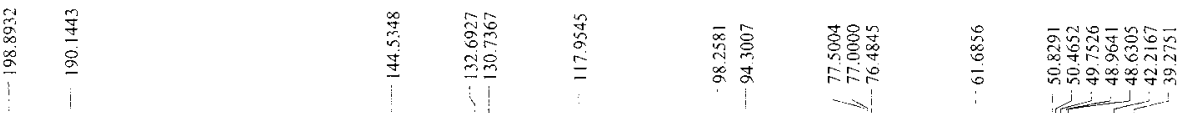
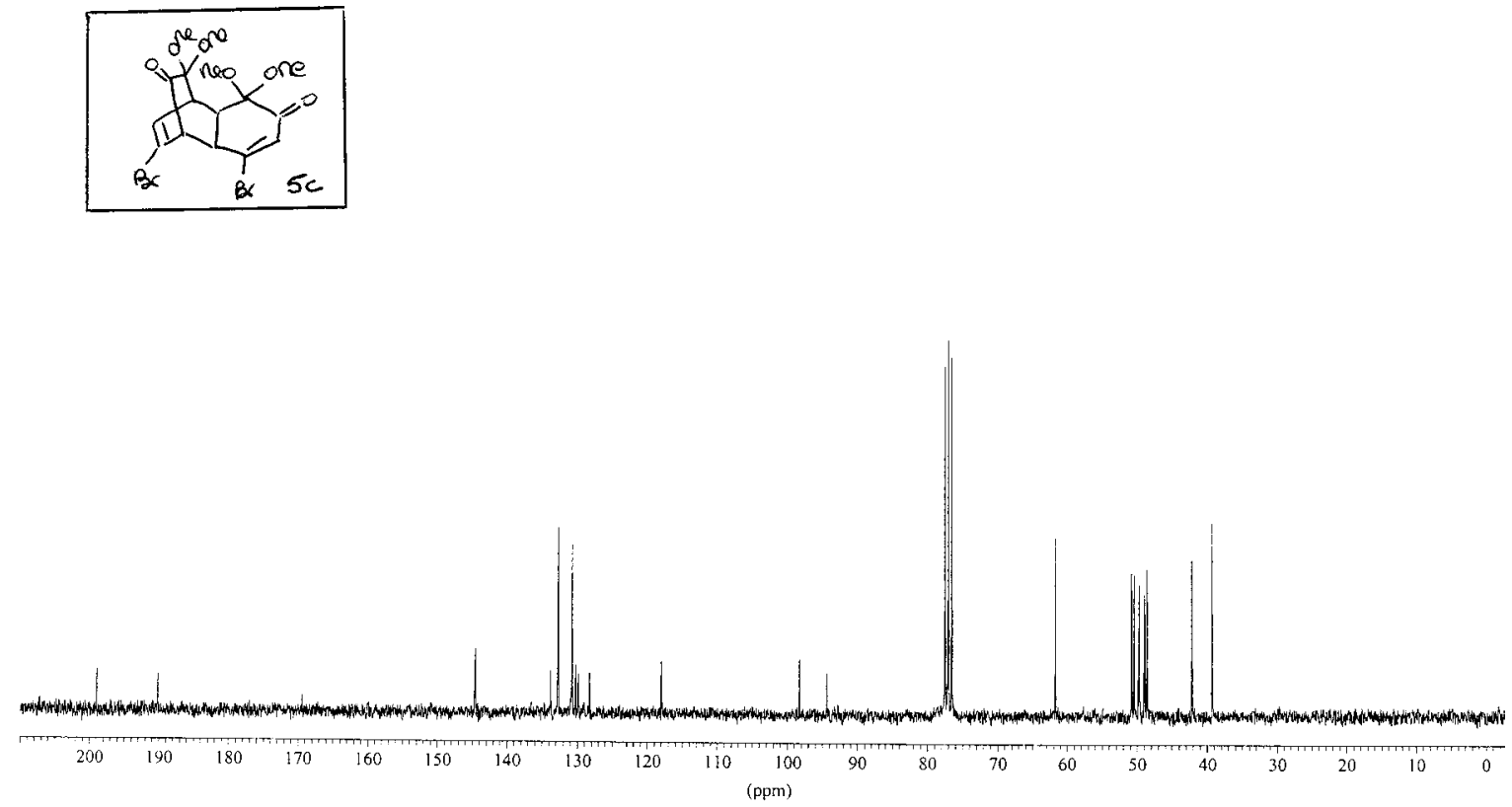

FB16CCOC13.25041338:DPX 300
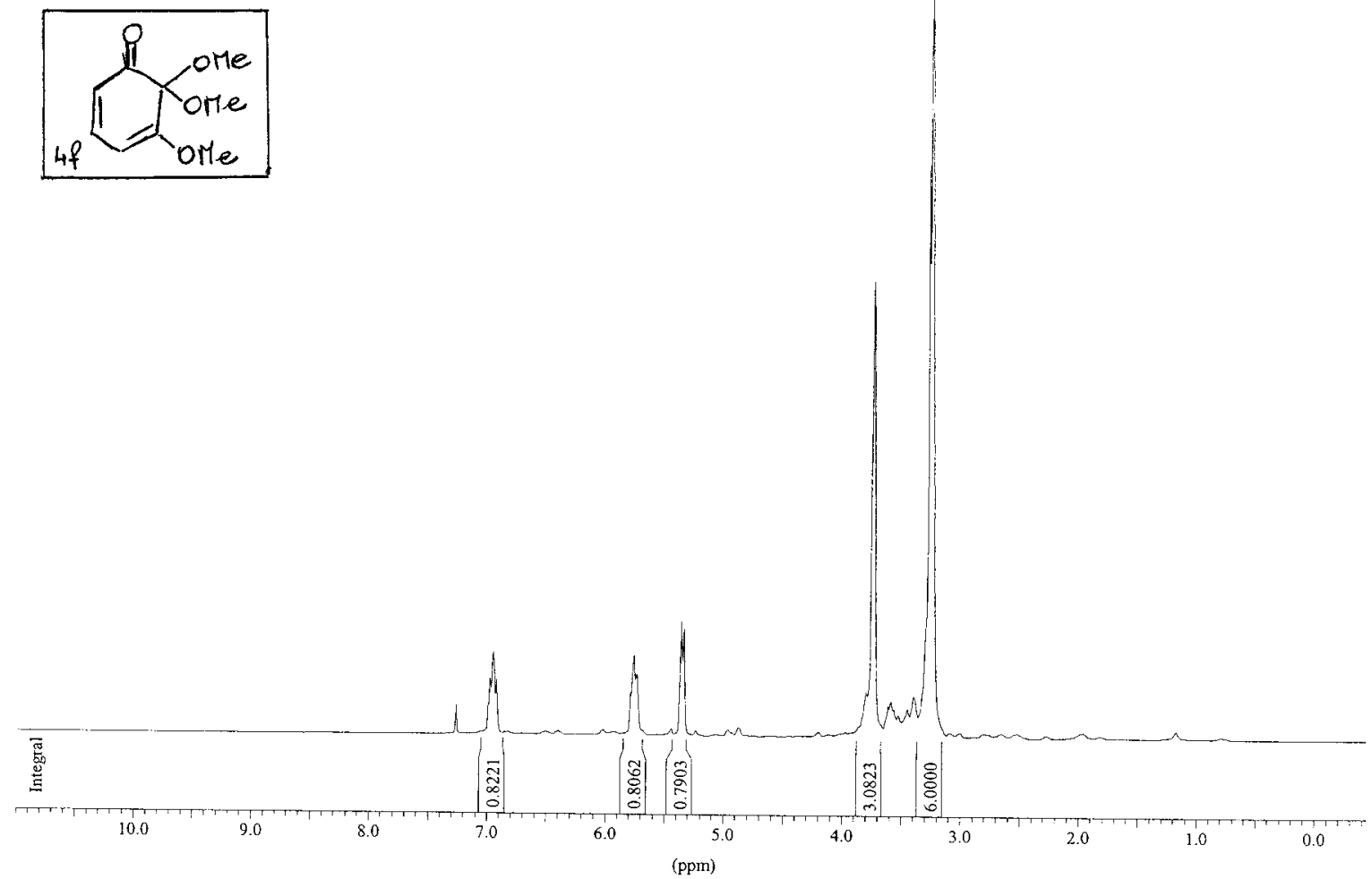
File Name : d: \2002-2 1 Idpx300 1 Peak Results saved in File: Nucleus SF OFFSET

SW_p

$S I$ : off : $300.130006 \mathrm{MHz}$

: $19.7643 \mathrm{ppm}$

: $6172.84 \mathrm{~Hz}$

: 16384

\section{Peak Picking Parameter}

Peak constant $\mathrm{PC}=$ 1.00

Noise

Sens. leve]

377

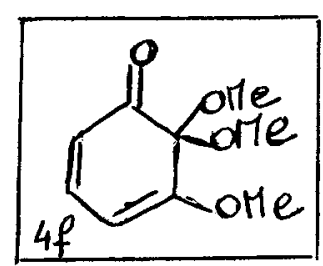

Peak Picking region

\begin{tabular}{ccrc} 
Start $(\mathrm{ppm} / \mathrm{Hz})$ & End $(\mathrm{ppm} / \mathrm{Hz})$ & MI $\left(\frac{\circ}{\circ}\right)$ & MAXI (\%) \\
\hline $3.24 / 973.7$ & $3.24 / 971.4$ & 99.33 & 101.61 \\
$3.84 / 1151.1$ & $3.60 / 1081.1$ & 50.23 & 70.14 \\
$5.43 / 1630.0$ & $5.22 / 1567.1$ & 9.16 & 26.58 \\
$5.86 / 1758.5$ & $5.52 / 1657.5$ & 4.80 & 19.74 \\
$7.01 / 2103.2$ & $6.83 / 2049.7$ & 6.05 & 17.25 \\
$7.34 / 2204.2$ & $7.14 / 2142.4$ & 2.94 & 12.27 \\
\hline
\end{tabular}

Peak Picking results

\begin{tabular}{rrrrrr} 
Peak Nr. Data Point & Frequency & \multicolumn{1}{c}{ PPM } & Intensity & \% Int. \\
\hline 1 & 9961 & 2178.94 & 7.2600 & 3869 & 3.9 \\
2 & 10189 & 2093.04 & 6.9738 & 7497 & 7.5 \\
3 & 10208 & 2085.88 & 6.9499 & 11042 & 11.1 \\
4 & 10233 & 2076.47 & 6.9186 & 7307 & 7.3 \\
5 & 11135 & 1736.63 & 5.7863 & 5712 & 5.7 \\
6 & 11159 & 1727.59 & 5.7561 & 10660 & 10.7 \\
7 & 11176 & 1721.18 & 5.7348 & 8265 & 8.3 \\
8 & 11477 & 1607.78 & 5.3569 & 15259 & 15.3 \\
9 & 11495 & 1600.99 & 5.3343 & 14194 & 14.3 \\
10 & 12774 & 1119.12 & 3.7288 & 60938 & 61.3 \\
11 & 13162 & 972.93 & 3.2417 & 99455 & 100.0 \\
\hline
\end{tabular}


IFB1 16-1/CDCI3/IN26-102/AC 250

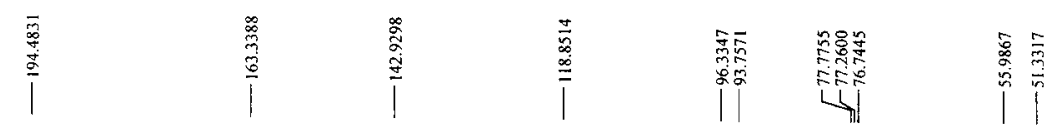

$$
\begin{aligned}
& \& \text { one } \\
& 1 \text { - Pone } \\
& \text { one }
\end{aligned}
$$

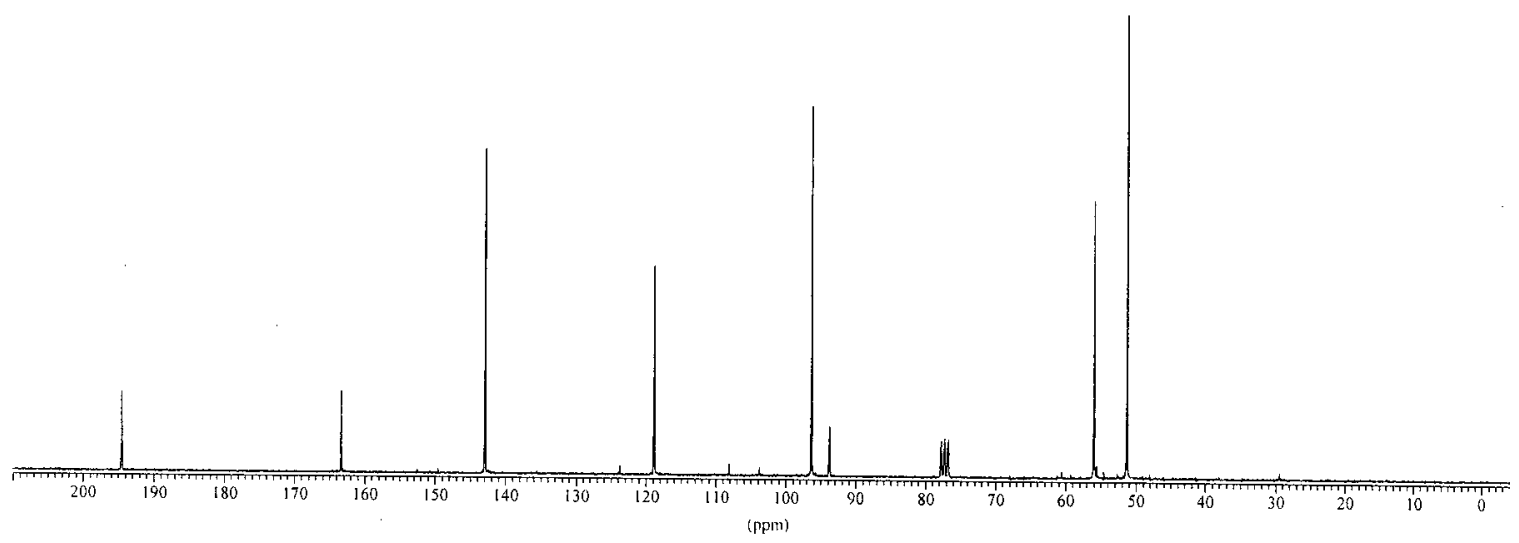

IFB 128.2/CDCl3/M113-101/AC 250
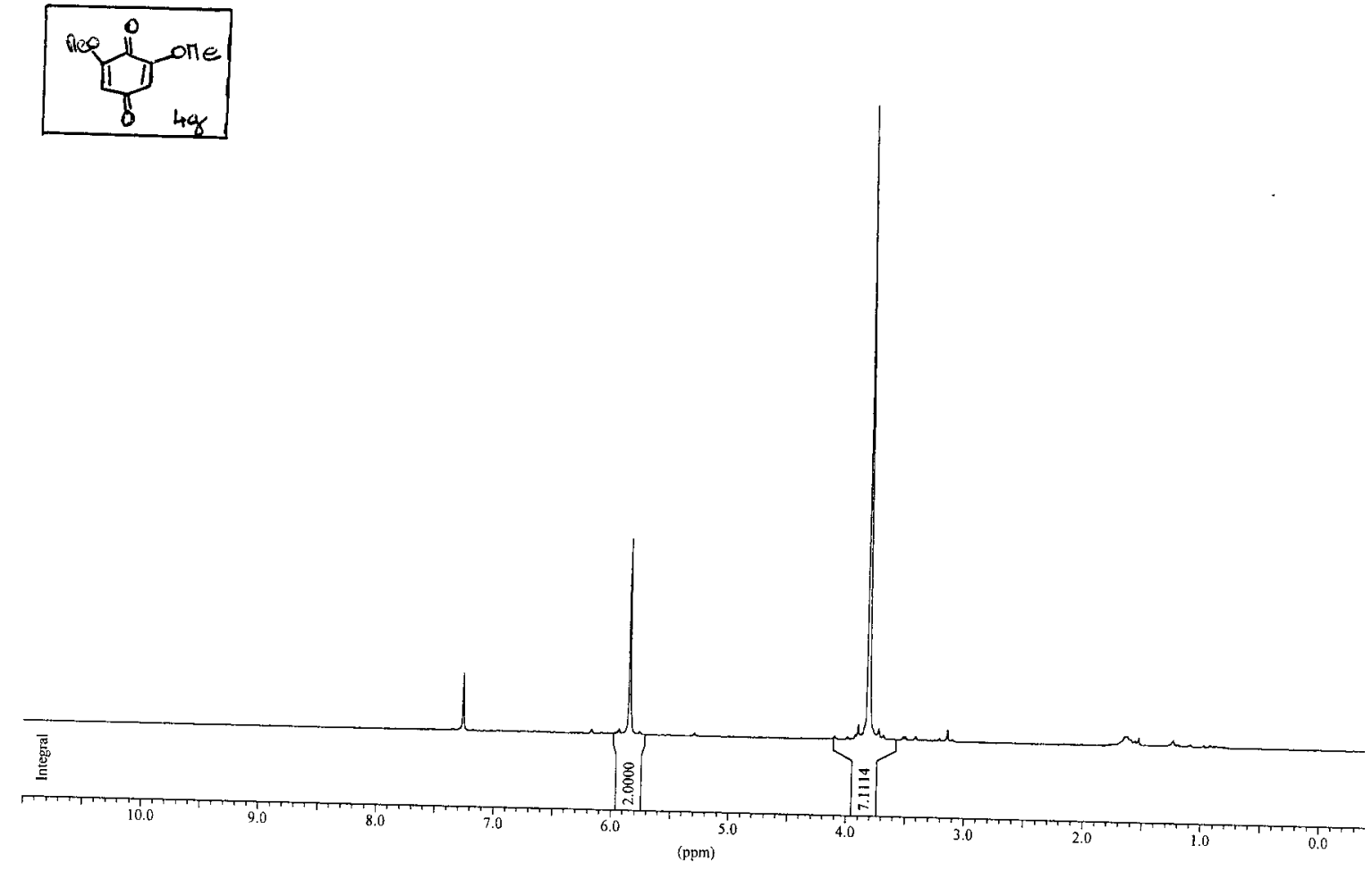


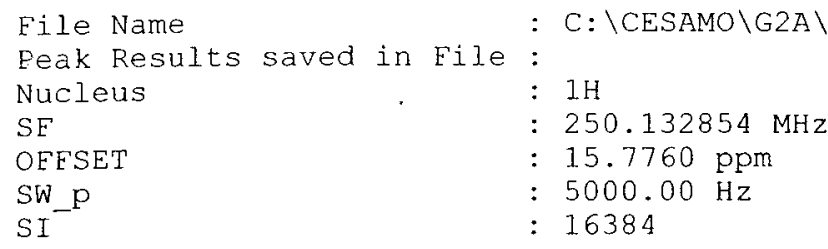

Peak Picking Parameter

$\begin{array}{lrr}\text { Peak constant } \mathrm{PC} & = & 1.00 \\ \text { Noise } & = & 1\end{array}$

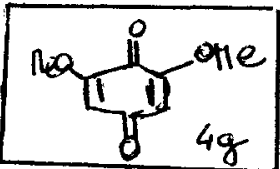

$=$

5

Peak Picking region

\begin{tabular}{rrrr} 
Start $(\mathrm{ppm} / \mathrm{Hz})$ & End $(\mathrm{ppm} / \mathrm{Hz})$ & MI (o) & \multicolumn{1}{c}{ MAXI (\%) } \\
\hline $7.63 / 1908.1$ & $5.34 / 1334.7$ & 7.46 & 42.76 \\
$5.98 / 1496.1$ & $1.66 / 416.1$ & 76.61 & 136.25 \\
\hline
\end{tabular}

Peak Picking results

\begin{tabular}{crrrrr} 
Peak Nr. Data Point & Frequency & PPM & Intensity & o. Int. \\
\hline 1 & 6980 & 1815.96 & 7.2600 & 1205 & 8.9 \\
2 & 8139 & 1462.27 & 5.8460 & 4137 & 30.7 \\
3 & 9805 & 953.84 & 3.8133 & 13483 & 100.0 \\
\hline
\end{tabular}




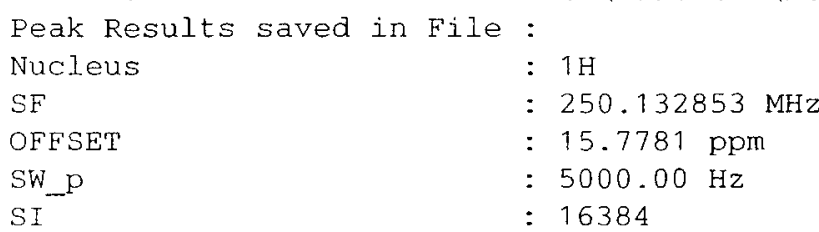

Peak Picking Parameter

$\begin{array}{llr}\text { Peak constant PC } & & 1.00 \\ \text { Noise level } & = & 3 \\ \text { Sens. level } & & 11\end{array}$

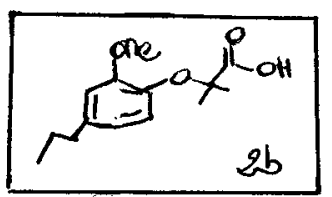

Peak Picking region

\begin{tabular}{crrr} 
Start $(\mathrm{ppm} / \mathrm{Hz})$ & End $(\mathrm{ppm} / \mathrm{Hz})$ & MI $(8)$ & MAXI $(8)$ \\
\hline $7.28 / 1822.0$ & $7.24 / 1811.9$ & 0.37 & 2.42 \\
$6.93 / 1734.4$ & $6.83 / 1708.1$ & 6.83 & 12.08 \\
$6.74 / 1686.2$ & $6.69 / 1674.6$ & 6.94 & 11.81 \\
$6.71 / 1678.2$ & $6.70 / 1674.9$ & 5.11 & 7.92 \\
$6.68 / 1671.5$ & $6.67 / 1667.6$ & 3.11 & 7.04 \\
$3.89 / 2973.6$ & $3.81 / 951.9$ & 40.57 & 94.75 \\
$2.61 / 653.5$ & $2.47 / 618.4$ & 3.37 & 19.76 \\
$1.68 / 419.7$ & $1.54 / 236.1$ & 1.81 & 13.19 \\
$0.99 / 248.2$ & $0.88 / 219.5$ & 5.08 & 61.16 \\
\hline
\end{tabular}

Peak Picking results

$\begin{array}{rrrrrr}\text { Peak Nr. Data Point } & \text { Frequency } & \text { PPM } & \text { Intensity } & \text { s Int. } \\ 1 & 6981 & 1816.18 & 7.2609 & 193 & 1.0 \\ 2 & 7272 & 1727.37 & 6.9058 & 1600 & 8.4 \\ 3 & 7298 & 1719.44 & 6.8741 & 2195 & 11.5 \\ 4 & 7422 & 1681.60 & 6.7228 & 1811 & 9.5 \\ 5 & 7437 & 1677.02 & 6.7045 & 1227 & 6.4 \\ 6 & 7463 & 1669.08 & 6.6728 & 806 & 4.2 \\ 7 & 9784 & 960.77 & 3.8410 & 11331 & 59.3 \\ 8 & 10829 & 641.86 & 2.5661 & 1197 & 6.3 \\ 9 & 10853 & 634.54 & 2.5368 & 1586 & 8.3 \\ 10 & 10879 & 626.60 & 2.5051 & 1354 & 7.1 \\ 11 & 11549 & 422.13 & 1.6876 & 202 & 1.1 \\ 12 & 11573 & 414.81 & 1.6584 & 766 & -4.0 \\ 13 & 11598 & 407.18 & 1.6279 & 1296 & -6.8 \\ 14 & 11623 & 399.55 & 1.5974 & 1307 & 6.8 \\ 15 & 11647 & 392.23 & 1.5681 & 838 & -4.4 \\ 16 & 11671 & 384.90 & 1.5388 & 268 & 1.4 \\ 17 & 11713 & 372.09 & 1.4876 & 19108 & 100.0 \\ 18 & 12155 & 237.20 & 0.9483 & 2819 & 14.8 \\ 19 & 12179 & 229.87 & 0.9190 & 5553 & 29.1 \\ 20 & 12203 & 222.55 & 0.8897 & 2362 & 12.4\end{array}$


IFA $12.3 / \mathrm{CDC}[3 / \mathrm{mu} 08-103 / \mathrm{C} 13 / \mathrm{AC} 250$

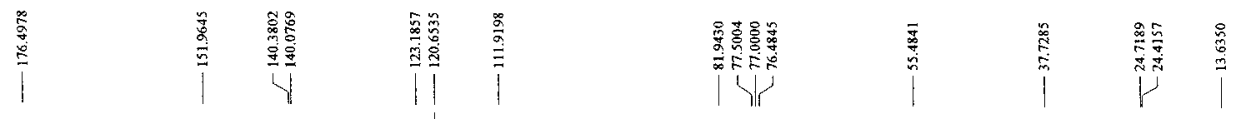

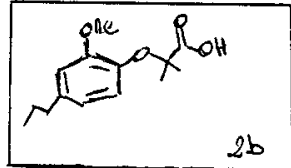

$2 b$

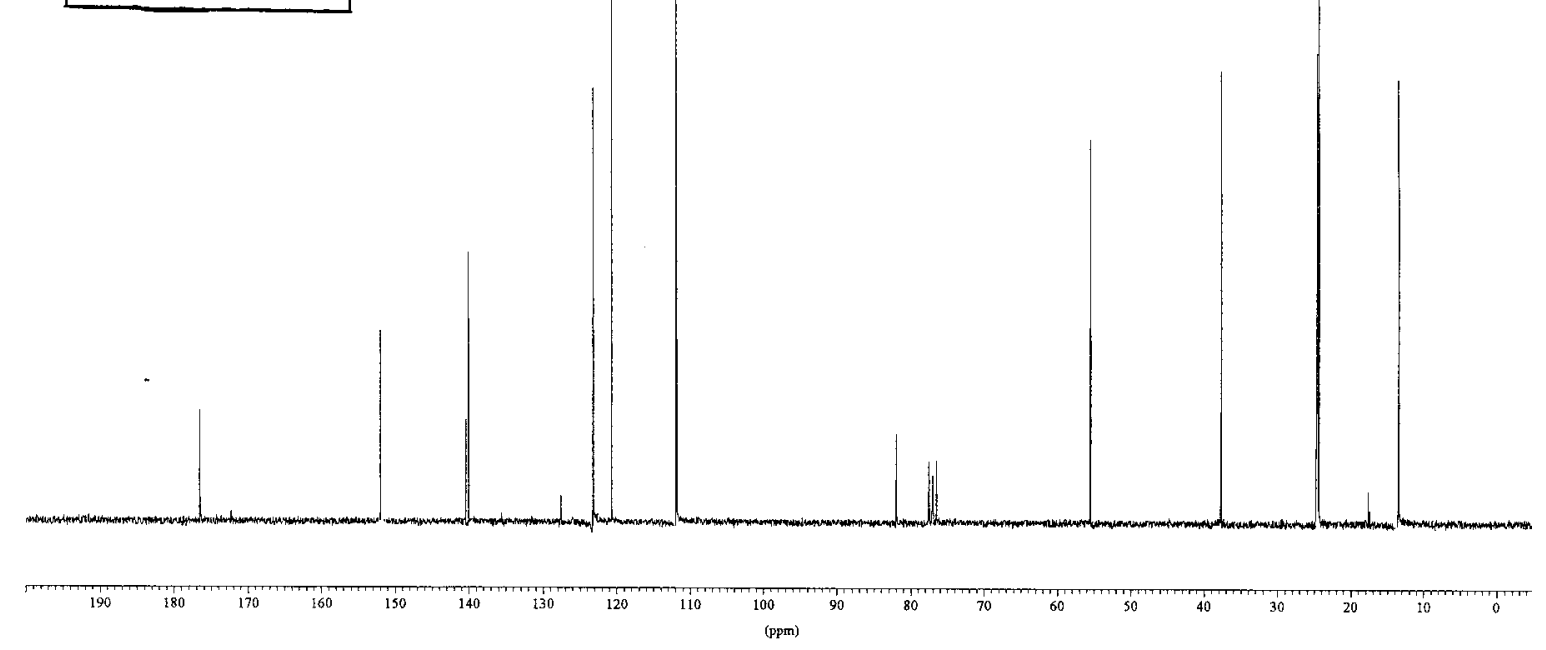

IFA20-2-3/CDC13/M123-130/AC 200

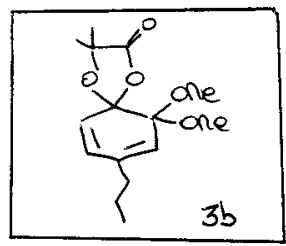

$3 b$

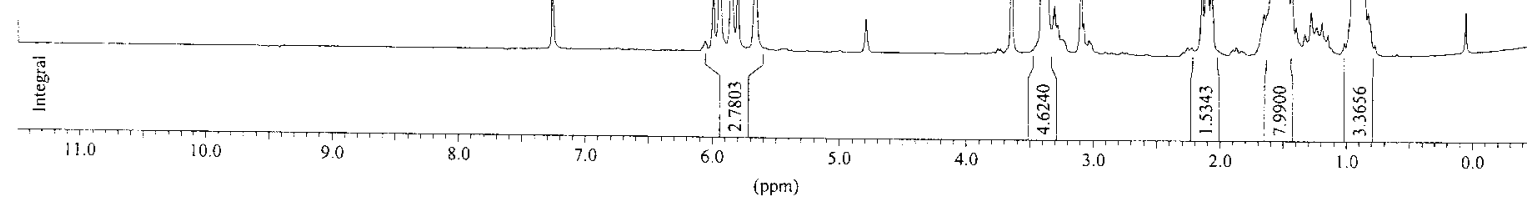


File Name

C: \WIN1D \SPC \ASP3000\MI23\130001.IR

Peak Results saved in File:

Nucleus

$: 1 \mathrm{H}$

SF

: $200.132341 \mathrm{MHz}$

OFFSET

$\mathrm{SI}_{\mathrm{SI}}$

$11.4988 \mathrm{ppm}$

: $2403.85 \mathrm{~Hz}$

: 8192

Peak Picking Parameter

$\begin{array}{llr}\text { Peak constant PC } & = & 1.00 \\ \text { Noise } & = & 6 \\ \text { Sens, level } & = & 2\end{array}$

6

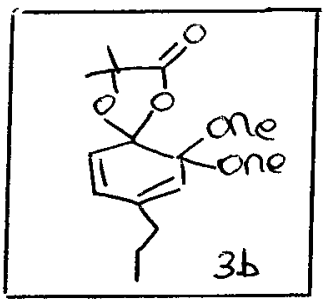

Peak Picking region

\begin{tabular}{|c|c|c|c|}
\hline Start $(\mathrm{ppm} / \mathrm{Hz})$ & End $(\mathrm{ppm} / \mathrm{Hz})$ & MI ( $\left.\frac{0}{2}\right)$ & $\operatorname{MAXI}\left(\frac{\circ}{5}\right)$ \\
\hline $8.05 / 1610.5$ & $6.99 / 1399.3$ & 14.71 & 26.72 \\
\hline $6.32 / 1265.5$ & $5.43 / 1085.9$ & 6.99 & 27.58 \\
\hline 737.9 & $2.86 /$ & 78.84 & 121.75 \\
\hline 470.2 & $1.90 /$ & 7.78 & 28.03 \\
\hline $1.15 /$ & $0.54 /$ & 18.42 & 57.90 \\
\hline 365.8 & $1.11 /$ & 74.38 & 114.54 \\
\hline 322.6 & $1.35 /$ & 6.52 & 27.39 \\
\hline 395.4 & $1.84 /$ & 86.97 & 92.29 \\
\hline
\end{tabular}

Peak Picking results

\begin{tabular}{ccrrrr} 
Peak Nr. Data Point & Frequency & PPM & Intensity & oInt. \\
\hline 1 & 2891 & 1452.96 & 7.2600 & 661 & 18.8 \\
2 & 3754 & 1199.72 & 5.9947 & 259 & 7.4 \\
3 & 3787 & 1190.04 & 5.9463 & 517 & 14.7 \\
4 & 3853 & 1170.67 & 5.8495 & 873 & 24.9 \\
5 & 3886 & 1160.99 & 5.8011 & 363 & 10.3 \\
6 & 3978 & 1133.99 & 5.6562 & 641 & 18.3 \\
7 & 5527 & 679.46 & 3.3950 & 3509 & 100.0 \\
8 & 5540 & 675.64 & 3.3760 & 3447 & 98.2 \\
9 & 6382 & 428.57 & 2.1414 & 336 & 9.6 \\
10 & 6407 & 421.23 & 2.1048 & 646 & 18.4 \\
11 & 6432 & 413.90 & 2.0681 & 434 & 12.4 \\
12 & 6768 & 315.30 & 1.5755 & 3446 & 98.2 \\
13 & 6792 & 308.26 & 1.5403 & 497 & 14.2 \\
14 & 6828 & 297.69 & 1.4875 & 3055 & 87.1 \\
15 & 6867 & 286.25 & 1.4303 & 335 & 9.5 \\
16 & 7194 & 190.30 & 0.9509 & 876 & 25.0 \\
17 & 7219 & 182.96 & 0.9142 & 1577 & 44.9 \\
18 & 7244 & 175.62 & 0.8775 & 794 & 22.6 \\
\hline
\end{tabular}


IFA 20.2.2A/CDC13/J605-113/AC 200

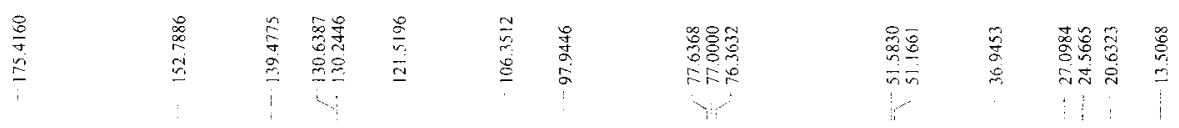
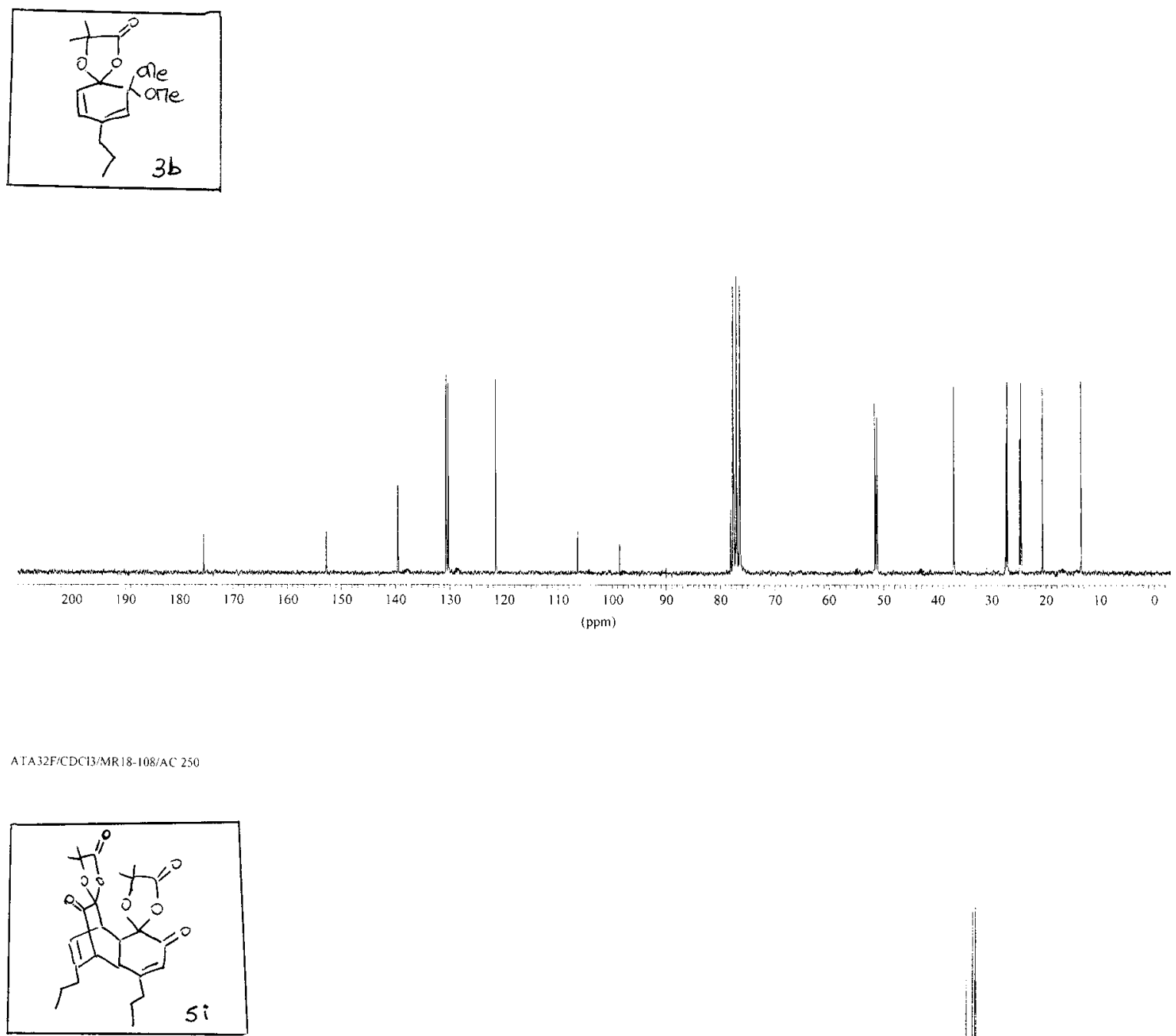

$5 i$

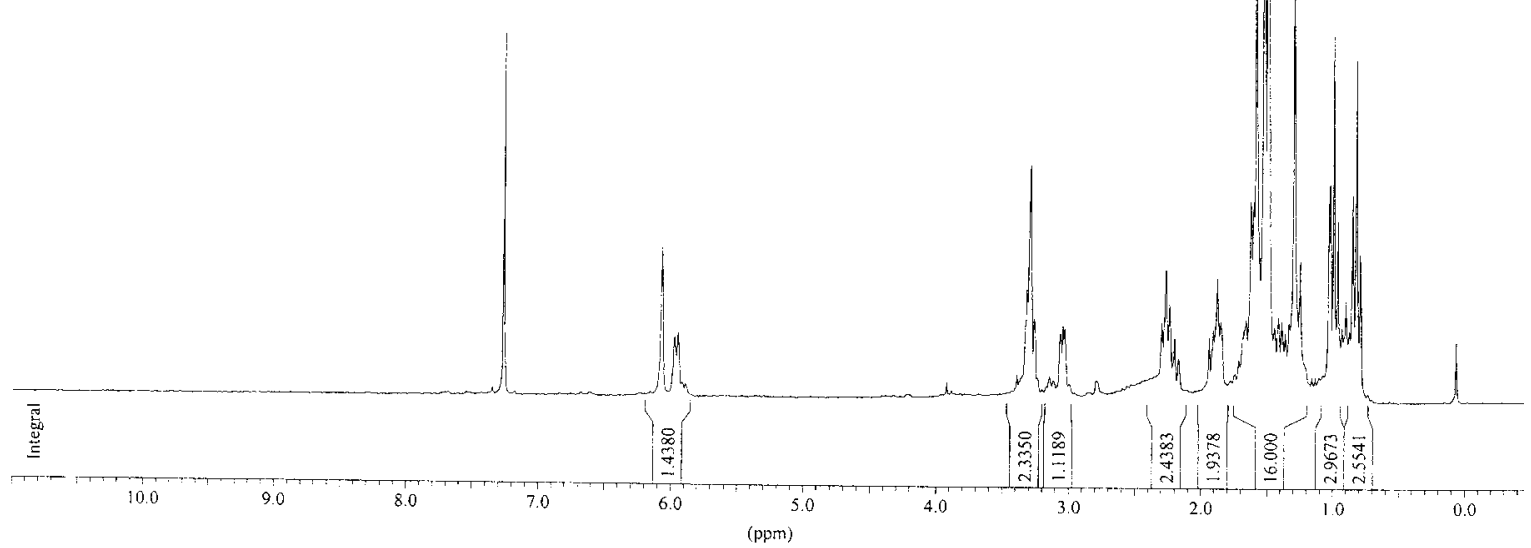


File Name

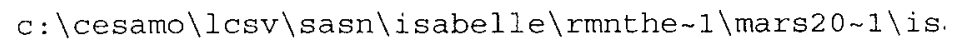
Peak Results saved in File: Nucleus $\mathrm{SF}$ OFFSET

$S W \_p$ $1 \mathrm{H}$ : $250.132854 \mathrm{MHz}$ : $15.7760 \mathrm{ppm}$ : $5000.00 \mathrm{~Hz}$ : 16384

Peak Picking Parameter

Peak constant $\mathrm{PC}=$ Noise

Sens. level

$=$
$=$

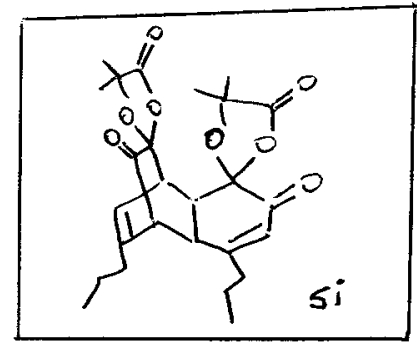

peak Picking region

\begin{tabular}{|c|c|c|c|}
\hline Start (ppm/Hz) & End $(\mathrm{ppm} / \mathrm{Hz})$ & $\mathrm{MI}\left(\frac{\circ}{0}\right)$ & $\operatorname{MAXI}(\%)$ \\
\hline $8.88 / 2220.0$ & $5.76 / 1440.6$ & 38.35 & 64.20 \\
\hline $6.14 / 1535.2$ & $5.94 / 1486.1$ & 18.12 & 21.54 \\
\hline $6.01 / 1502.2$ & $5.88 / 1469.6$ & 6.16 & 11.57 \\
\hline $3.44 / 859.5$ & $3.14 / 784.5$ & 9.02 & 37.54 \\
\hline 777.5 & 746.6 & 7.58 & 13.24 \\
\hline 594.3 & 537.9 & 4.49 & 21.48 \\
\hline 508.9 & 452.4 & 6.04 & 19.94 \\
\hline 421.9 & $1.14 /$ & 51.03 & 112.17 \\
\hline 222.9 & $0.74 /$ & 16.91 & 56.96 \\
\hline
\end{tabular}

Peak Picking results

\begin{tabular}{rrrrrr} 
Peak Nr. Data Point & Frequency & \multicolumn{1}{c}{ PPM } & Intensity & \multicolumn{1}{c}{ \%nt. } \\
\hline 1 & 6980 & 1815.96 & 7.2600 & 777 & 51.2 \\
2 & 7959 & 1517.20 & 6.0656 & 318 & 21.0 \\
3 & 8036 & 1493.70 & 5.9716 & 125 & 8.3 \\
4 & 8060 & 1486.37 & 5.9423 & 135 & 8.9 \\
5 & 10216 & 828.42 & 3.3119 & 235 & 15.5 \\
6 & 10238 & 821.70 & 3.2851 & 502 & 33.1 \\
7 & 10261 & 814.68 & 3.2570 & 172 & 11.4 \\
8 & 10266 & 813.16 & 3.2509 & 158 & 10.4 \\
9 & 10421 & 765.85 & 3.0618 & 141 & 9.3 \\
10 & 10439 & 760.36 & 3.0398 & 156 & 10.3 \\
11 & 10449 & 757.31 & 3.0276 & 151 & 10.0 \\
12 & 11050 & 573.90 & 2.2944 & 165 & 10.9 \\
13 & 11076 & 565.96 & 2.2627 & 280 & 18.5 \\
14 & 11098 & 559.25 & 2.2358 & 204 & 13.5 \\
15 & 11129 & 549.79 & 2.1980 & 131 & 8.6 \\
16 & 11156 & 541.55 & 2.1651 & 89 & 5.9 \\
17 & 11344 & 484.18 & 1.9357 & 133 & 8.7 \\
18 & 11369 & 476.55 & 1.9052 & 151 & 9.9 \\
19 & 11394 & 468.92 & 1.8747 & 262 & 17.3 \\
20 & 11418 & 461.59 & 1.8454 & 169 & 11.1 \\
21 & 11603 & 405.14 & 1.6197 & 427 & 28.2 \\
22 & 11621 & 399.64 & 1.5977 & 426 & 28.1 \\
23 & 11636 & 395.07 & 1.5794 & 1302 & 85.8 \\
24 & 11686 & 379.81 & 1.5184 & 1494 & 98.5 \\
25 & 11710 & 372.48 & 1.4891 & 1517 & 100.0 \\
26 & 11875 & 322.13 & 1.2878 & 1138 & 75.0 \\
27 & 12088 & 257.13 & 1.0280 & 423 & 27.9
\end{tabular}




\begin{tabular}{llllll}
28 & 12097 & 254.38 & 1.0170 & 464 & 30.6 \\
29 & 12121 & 247.06 & 0.9877 & 785 & 51.7 \\
30 & 12145 & 239.73 & 0.9584 & 384 & 25.3 \\
31 & 12236 & 211.96 & 0.8474 & 440 & 29.0 \\
32 & 12260 & 204.64 & 0.8181 & 732 & 48.2 \\
33 & 12284 & 197.31 & 0.7888 & 314 & 20.7 \\
\hline
\end{tabular}

ATA32/CDCB3/19030426/DPX300

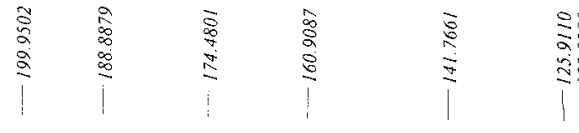

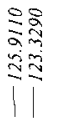
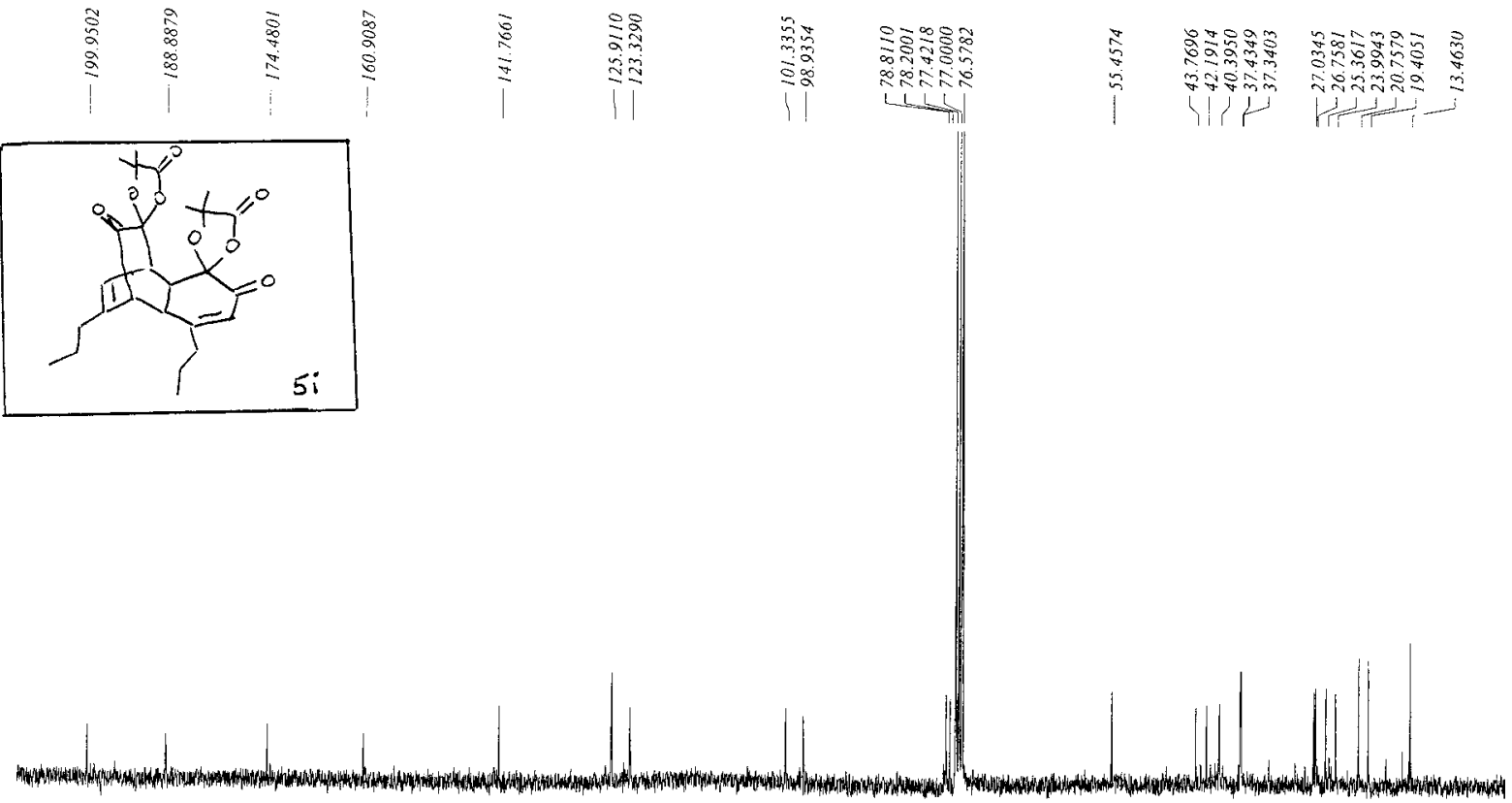

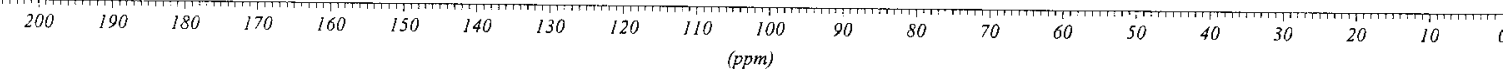




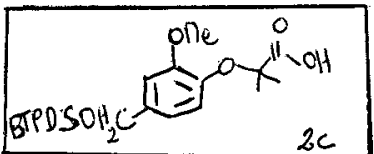

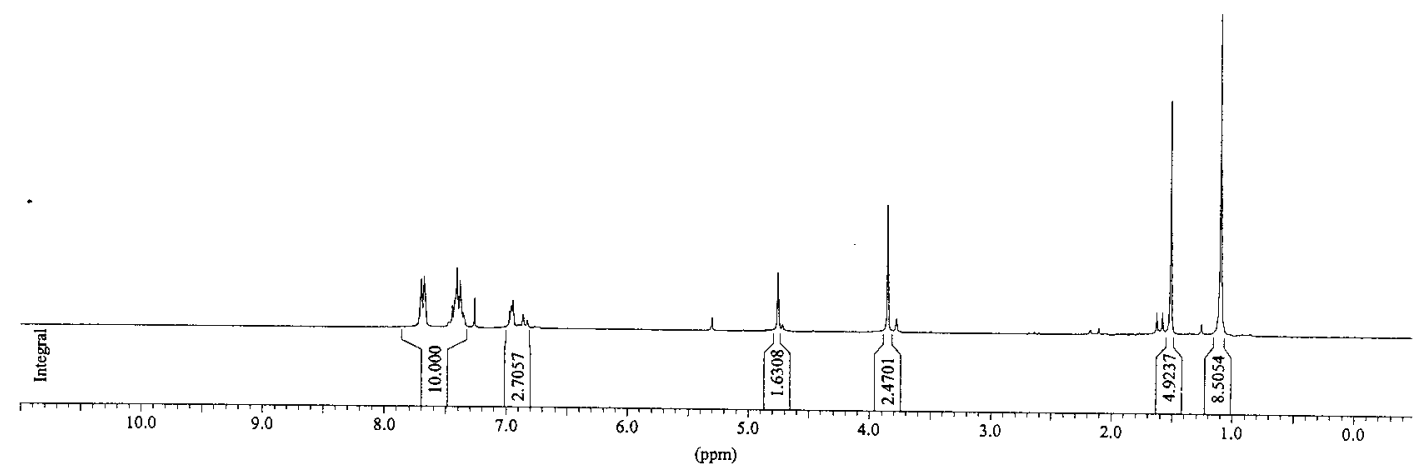


File Name

$\mathrm{d}: \backslash 2000-2 \sim 1 \backslash$ rmndea $1 \backslash$ avri12 1 1 ap02\115001.1R

Peak Results saved in File:

Nucleus

$\mathrm{SF}$

OFFSET

SW_P

SI

$: 1 \mathrm{H}$

: $200.132341 \mathrm{MHz}$

: $11.4988 \mathrm{ppm}$

: $2403.85 \mathrm{~Hz}$

: 8192

Peak Picking Parameter

Peak constant $\mathrm{PC}=$

Noise

1.00

$=$

Sens. level

4

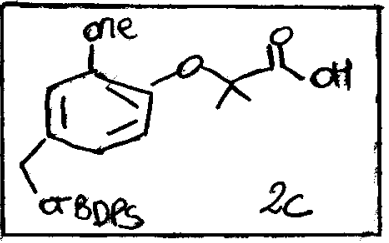

\section{Peak Picking region}

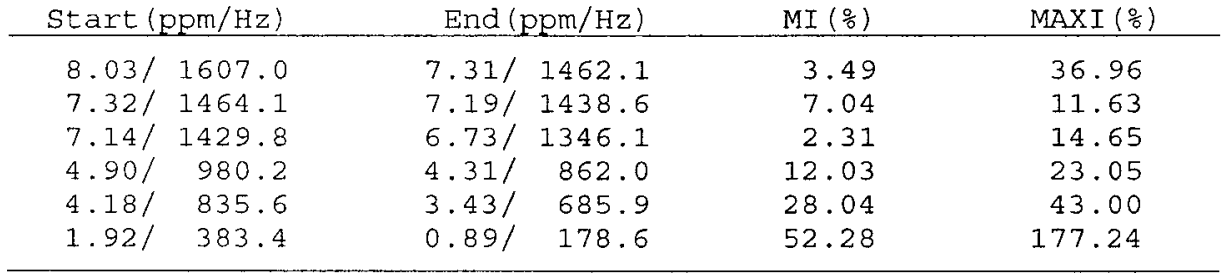

Peak Picking results

\begin{tabular}{rrrrrr} 
Peak Nr. Data Point & Frequency & PPM & Intensity & oInt. \\
\hline 1 & 2591 & 1540.99 & 7.6999 & 952 & 15.6 \\
2 & 2611 & 1535.12 & 7.6705 & 994 & 16.3 \\
3 & 2617 & 1533.36 & 7.6617 & 1144 & 18.8 \\
4 & 2765 & 1489.93 & 7.4447 & 383 & 6.3 \\
5 & 2788 & 1483.18 & 7.4110 & 1538 & 25.3 \\
6 & 2813 & 1475.85 & 7.3744 & 1023 & 16.8 \\
7 & 2832 & 1470.27 & 7.3465 & 334 & 5.5 \\
8 & 2891 & 1452.96 & 7.2600 & 533 & 8.8 \\
9 & 3083 & 1396.62 & 6.9785 & 297 & 4.9 \\
10 & 3102 & 1391.05 & 6.9506 & 531 & 8.7 \\
11 & 3110 & 1388.70 & 6.9389 & 672 & 11.0 \\
12 & 3165 & 1372.56 & 6.8583 & 327 & 5.4 \\
13 & 3193 & 1364.34 & 6.8172 & 170 & 2.8 \\
14 & 4603 & 950.59 & 4.7498 & 1202 & 19.8 \\
15 & 5218 & 770.13 & 3.8481 & 2386 & 39.2 \\
16 & 6809 & 303.27 & 1.5153 & 4375 & 71.9 \\
17 & 7089 & 221.11 & 1.1048 & 6084 & 100.0 \\
\hline
\end{tabular}


IFA. 28.2/CDCI3/AR05-1 10/AC 250

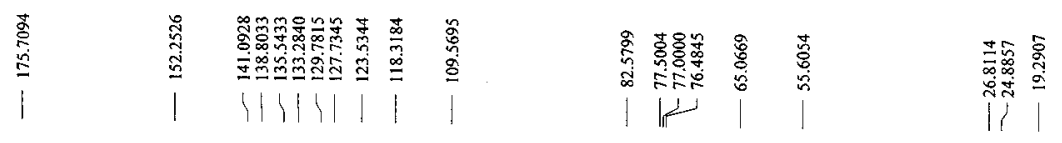

BTPSSOHL

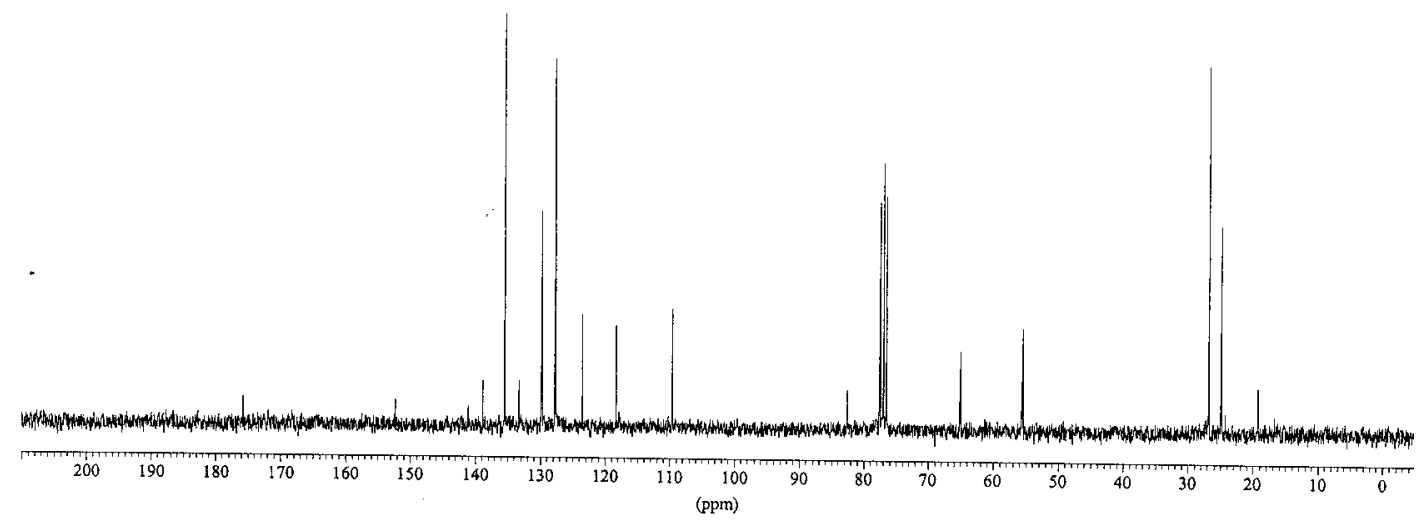


gpa 23-1/CDCi3/04070222/DPx 300
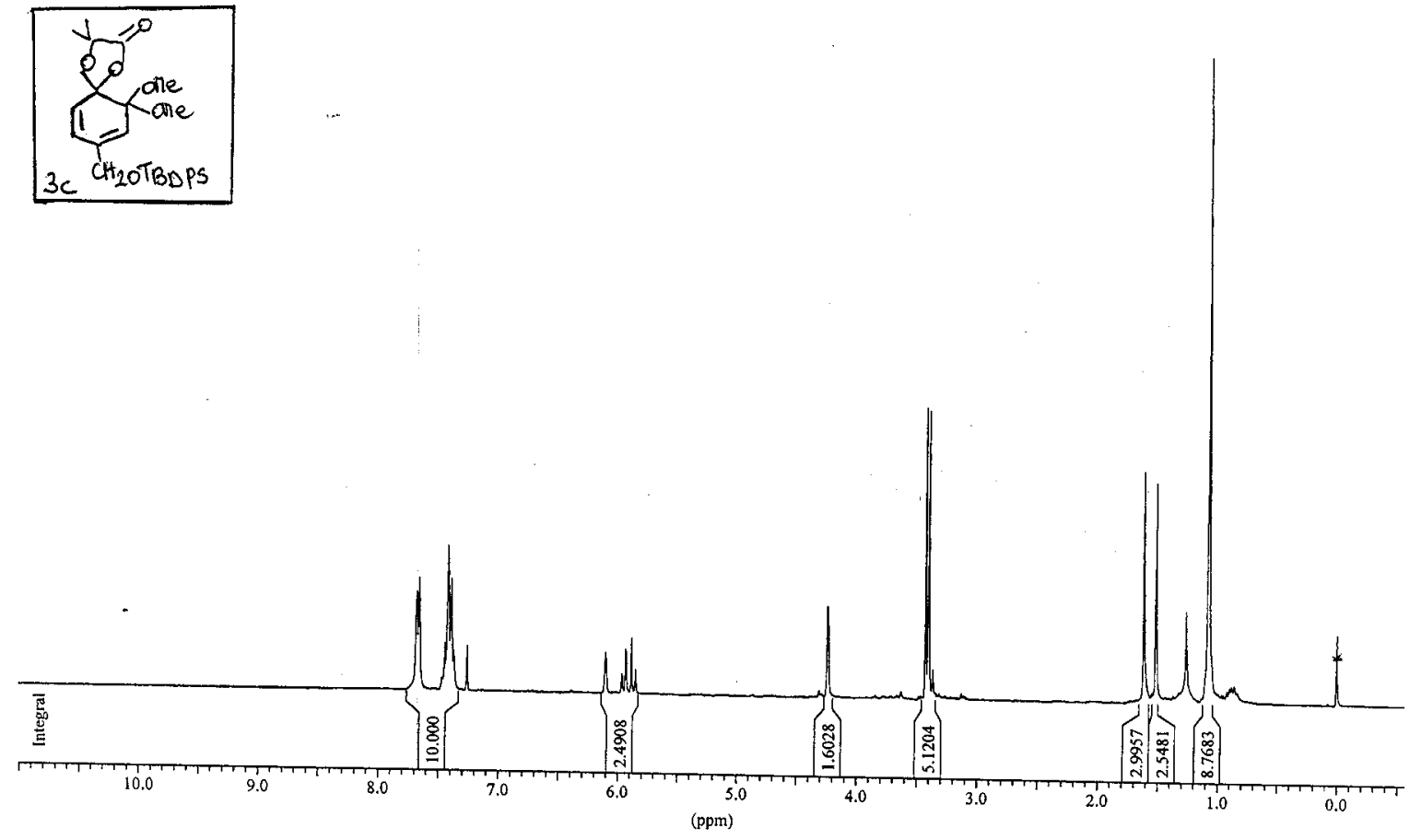
File Name

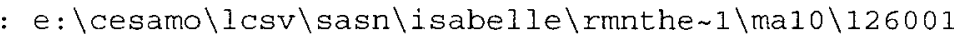

Peak Results saved in File:

Nucleus

SF

$: 1 \mathrm{H}$

OFFSET

SW_P

: $250.132855 \mathrm{MHz}$

: $15.7723 \mathrm{ppm}$

: $5000.00 \mathrm{~Hz}$

: 16384

Peak Picking Parameter

Peak constant $\mathrm{PC}=$

1.00

Noise

$\begin{array}{lr}= & 1.00 \\ & 1\end{array}$

$3 c$

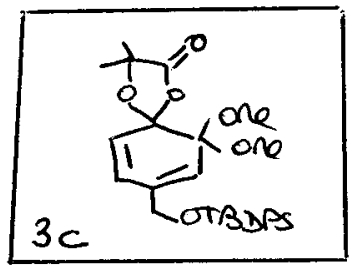

Sens. level

2

Peak Picking region

\begin{tabular}{|c|c|c|c|c|c|}
\hline \multicolumn{2}{|c|}{ Start $(\mathrm{ppm} / \mathrm{Hz})$} & \multicolumn{2}{|c|}{ End $(\mathrm{ppm} / \mathrm{Hz})$} & \multirow{2}{*}{$\frac{M I\left(\frac{\circ}{0}\right)}{26.84}$} & \multirow{2}{*}{$\frac{\operatorname{MAXI}\left(\frac{\circ}{6}\right)}{42.85}$} \\
\hline $3.63 /$ & 906.8 & $2.66 /$ & 664.2 & & \\
\hline $1.72 /$ & 429.9 & 1.041 & 259.6 & 23.97 & 37.52 \\
\hline $4.70 /$ & 1175.1 & 4.031 & 1009.1 & 11.85 & 17.60 \\
\hline $6.21 /$ & 1554.4 & 5.531 & 1383.8 & 1.18 & 8.78 \\
\hline $8.05 /$ & 2014.3 & $6.96 /$ & 1741.8 & 4.88 & 30.95 \\
\hline $2.02 /$ & 506.5 & 0.491 & 123.2 & 82.41 & 120.18 \\
\hline
\end{tabular}

Peak Picking results

\begin{tabular}{crrrrr} 
Peak Nr. Data Point & Frequency & PPM & Intensity & \%Int. \\
\hline 1 & 6631 & 1921.56 & 7.6821 & 654 & 12.9 \\
2 & 6651 & 1915.45 & 7.6577 & 708 & 14.0 \\
3 & 6655 & 1914.23 & 7.6529 & 695 & 13.8 \\
4 & 6824 & 1862.66 & 7.4467 & 358 & 7.1 \\
5 & 6845 & 1856.25 & 7.4210 & 1201 & 23.8 \\
6 & 6869 & 1848.92 & 7.3918 & 827 & 16.4 \\
7 & 6887 & 1843.43 & 7.3698 & 329 & 6.5 \\
8 & 6977 & 1815.96 & 7.2600 & 314 & 6.2 \\
9 & 7921 & 1527.88 & 6.1083 & 259 & 5.1 \\
10 & 7926 & 1526.35 & 6.1022 & 256 & 5.1 \\
11 & 8035 & 1493.09 & 5.9692 & 115 & 2.3 \\
12 & 8067 & 1483.32 & 5.9301 & 298 & 5.9 \\
13 & 8071 & 1482.10 & 5.9253 & 273 & 5.4 \\
14 & 8102 & 1472.64 & 5.8874 & 387 & 7.6 \\
15 & 8134 & 1462.88 & 5.8484 & 139 & 2.8 \\
16 & 9452 & 1060.65 & 4.2404 & 620 & 12.3 \\
17 & 10118 & 857.41 & 3.4278 & 1911 & 37.8 \\
18 & 10139 & 851.00 & 3.4022 & 1881 & 37.2 \\
19 & 11602 & 404.53 & 1.6172 & 1612 & 31.9 \\
20 & 11685 & 379.20 & 1.5160 & 1572 & 31.1 \\
21 & 12047 & 268.72 & 1.0743 & 5056 & 100.0 \\
\hline
\end{tabular}


gpa 23-1/CDCl3//C13/04070222/DPx 300

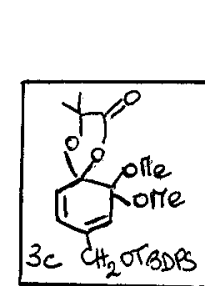

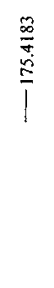
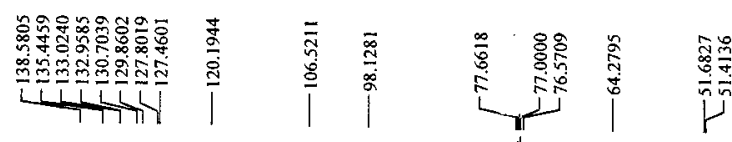

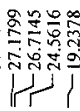

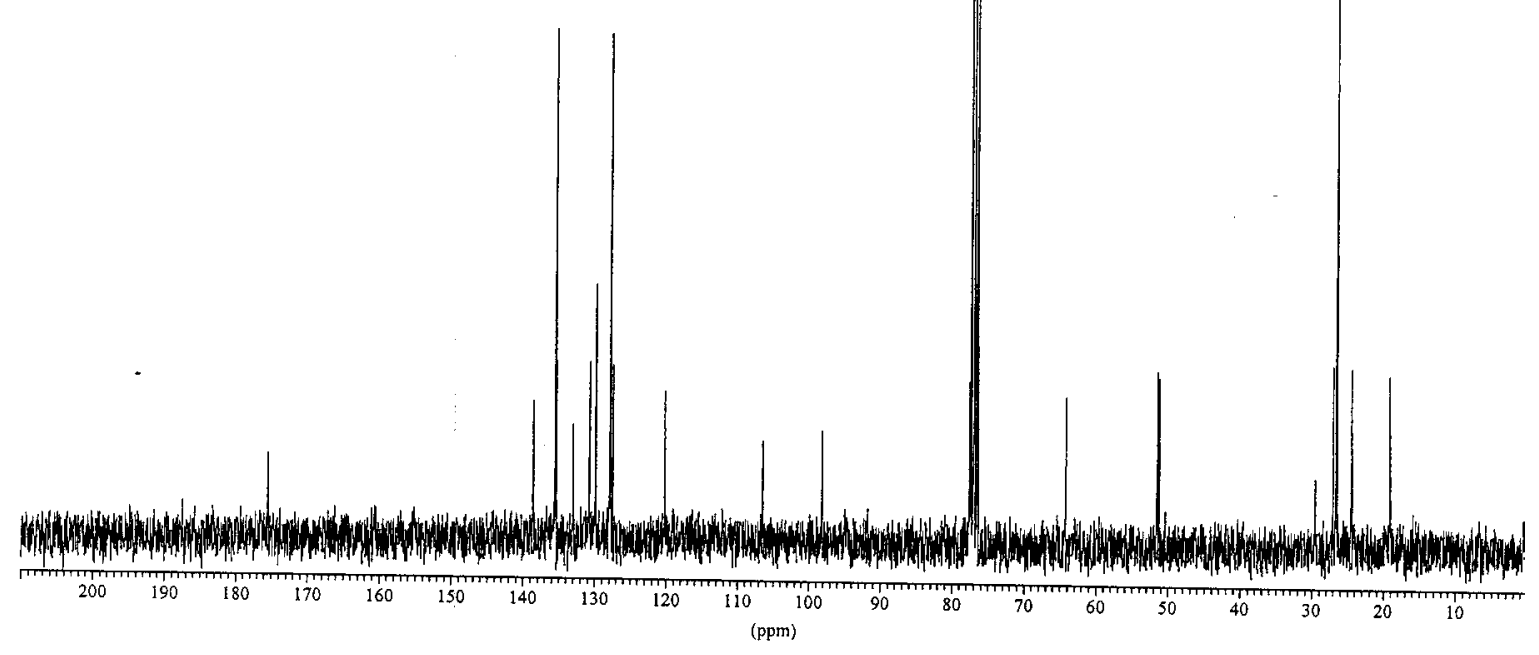

$$
\text { Corsops 2d }
$$

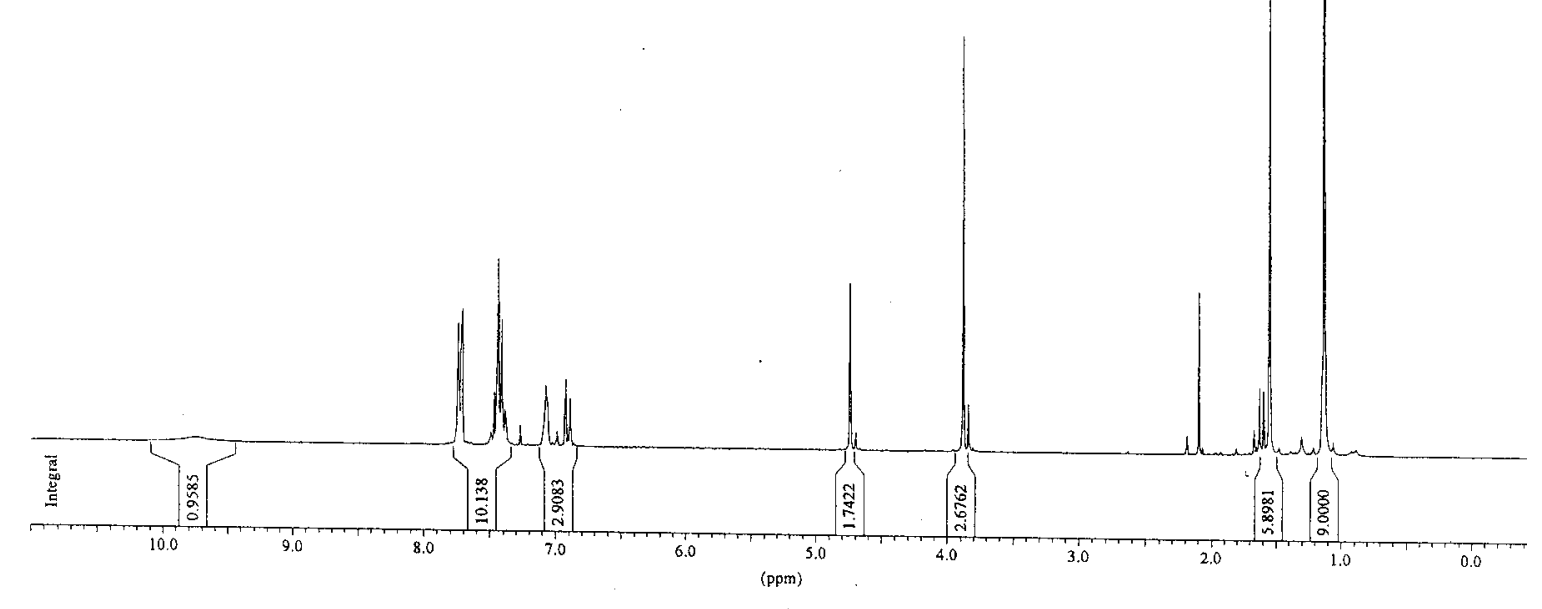




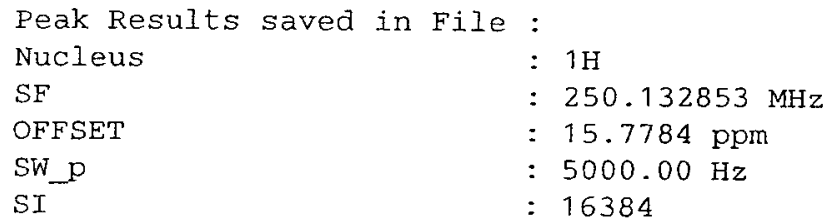

Peak Picking Parameter

Peak constant $\mathrm{PC}=\quad 1.00$

Noise $=3$

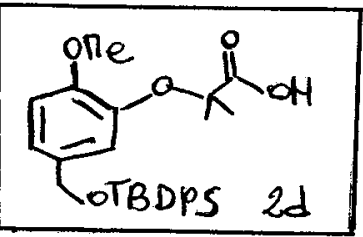

Sens. level = 13

Peak Picking region

\begin{tabular}{rrrr} 
Start $(\mathrm{ppm} / \mathrm{Hz})$ & End $(\mathrm{ppm} / \mathrm{Hz})$ & MI $\left(\frac{\circ}{6}\right)$ & MAXI (o) \\
\hline $7.76 / 1939.9$ & $7.74 / 1935.9$ & 9.24 & 13.81 \\
$7.74 / 1937.1$ & $7.73 / 1933.5$ & 12.95 & 14.64 \\
$7.72 / 1931.0$ & $7.69 / 1922.8$ & 13.18 & 15.98 \\
$7.73 / 1934.4$ & $7.73 / 1933.2$ & 8.02 & 9.01 \\
$7.16 / 1791.2$ & $7.01 / 1752.5$ & 2.95 & 8.02 \\
$6.97 / 1743.0$ & $6.76 / 1689.9$ & 1.32 & 8.54 \\
$6.91 / 1727.8$ & $6.86 / 1717.1$ & 0.49 & 1.61 \\
$7.00 / 1749.7$ & $6.98 / 1745.2$ & 1.40 & 2.25 \\
$10.35 / 2589.3$ & $9.05 / 2262.7$ & -0.13 & 8.18 \\
\hline
\end{tabular}

Peak Picking results

\begin{tabular}{rrrrrr} 
Peak Nx. Data Point & Frequency & PPM & Intensity & : Int. \\
\hline 1 & 4940 & 2439.13 & 9.7514 & 221 & 0.4 \\
2 & 6587 & 1936.51 & 7.7419 & 5572 & 11.1 \\
3 & 6590 & 1935.59 & 7.7383 & 6836 & 13.6 \\
4 & 6592 & 1934.98 & 7.7358 & 7117 & 14.2 \\
5 & 6609 & 1929.80 & 7.7151 & 6767 & 13.5 \\
6 & 6611 & 1929.18 & 7.7126 & 6780 & 13.5 \\
7 & 6616 & 1927.66 & 7.7065 & 7946 & 15.9 \\
8 & 6981 & 1816.27 & 7.2612 & 1171 & 2.3 \\
9 & 7125 & 1772.32 & 7.0855 & 1874 & 3.7 \\
10 & 7136 & 1768.97 & 7.0721 & 3012 & 6.0 \\
11 & 7140 & 1767.75 & 7.0672 & 3553 & 7.1 \\
12 & 7142 & 1767.14 & 7.0648 & 3480 & 6.9 \\
13 & 7147 & 1765.61 & 7.0587 & 2824 & 5.6 \\
14 & 7208 & 1746.99 & 6.9843 & 893 & 1.8 \\
15 & 7261 & 1730.82 & 6.9196 & 3895 & 7.8 \\
16 & 7274 & 1726.85 & 6.9037 & 636 & 1.3 \\
17 & 7290 & 1721.97 & 6.8842 & 2740 & 5.5 \\
18 & 9049 & 1185.77 & 4.7381 & 9789 & 19.5 \\
19 & 9752 & 970.63 & 3.8804 & 24371 & 48.6 \\
20 & 11661 & 388.05 & 1.5514 & 33417 & 66.7
\end{tabular}




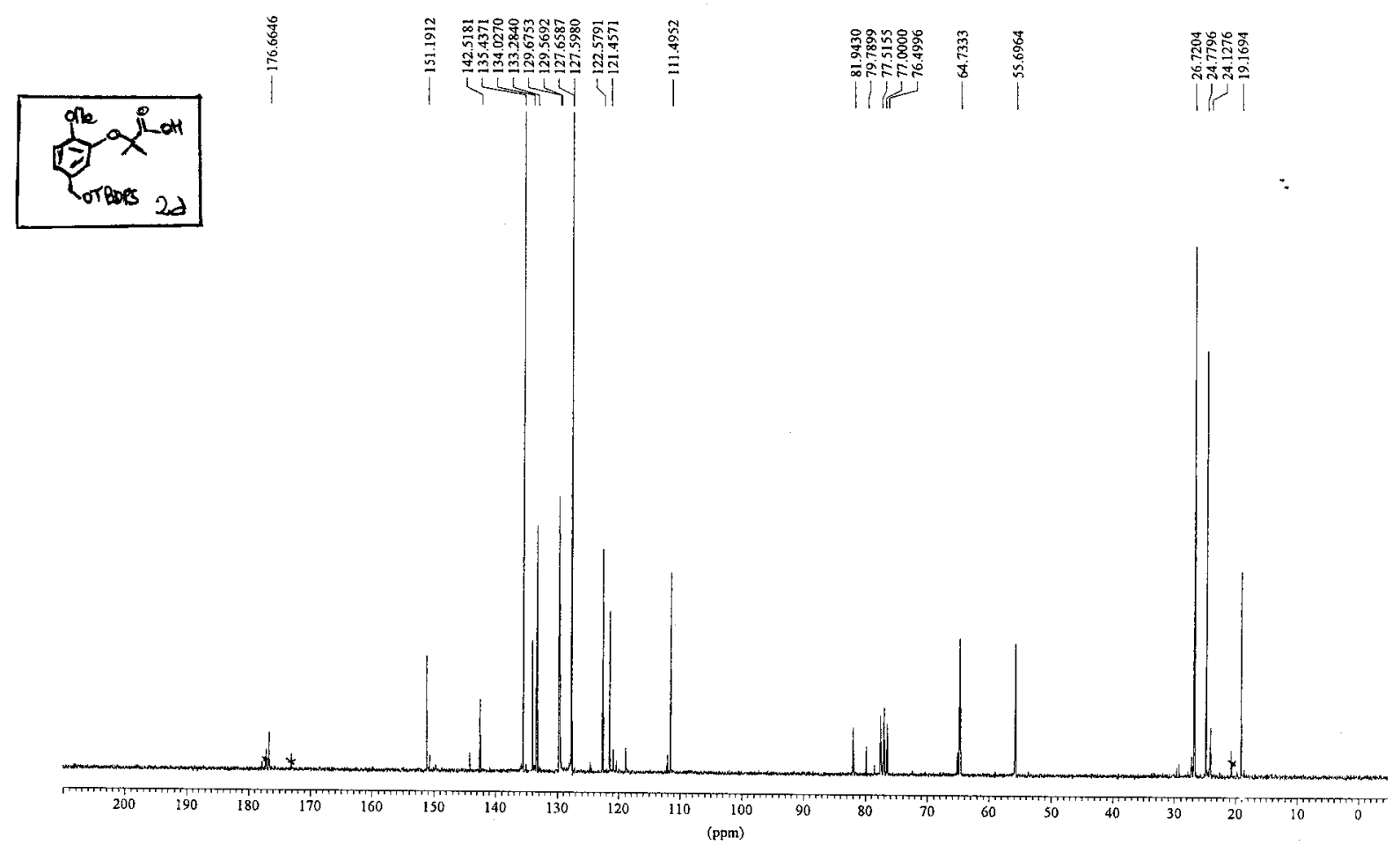



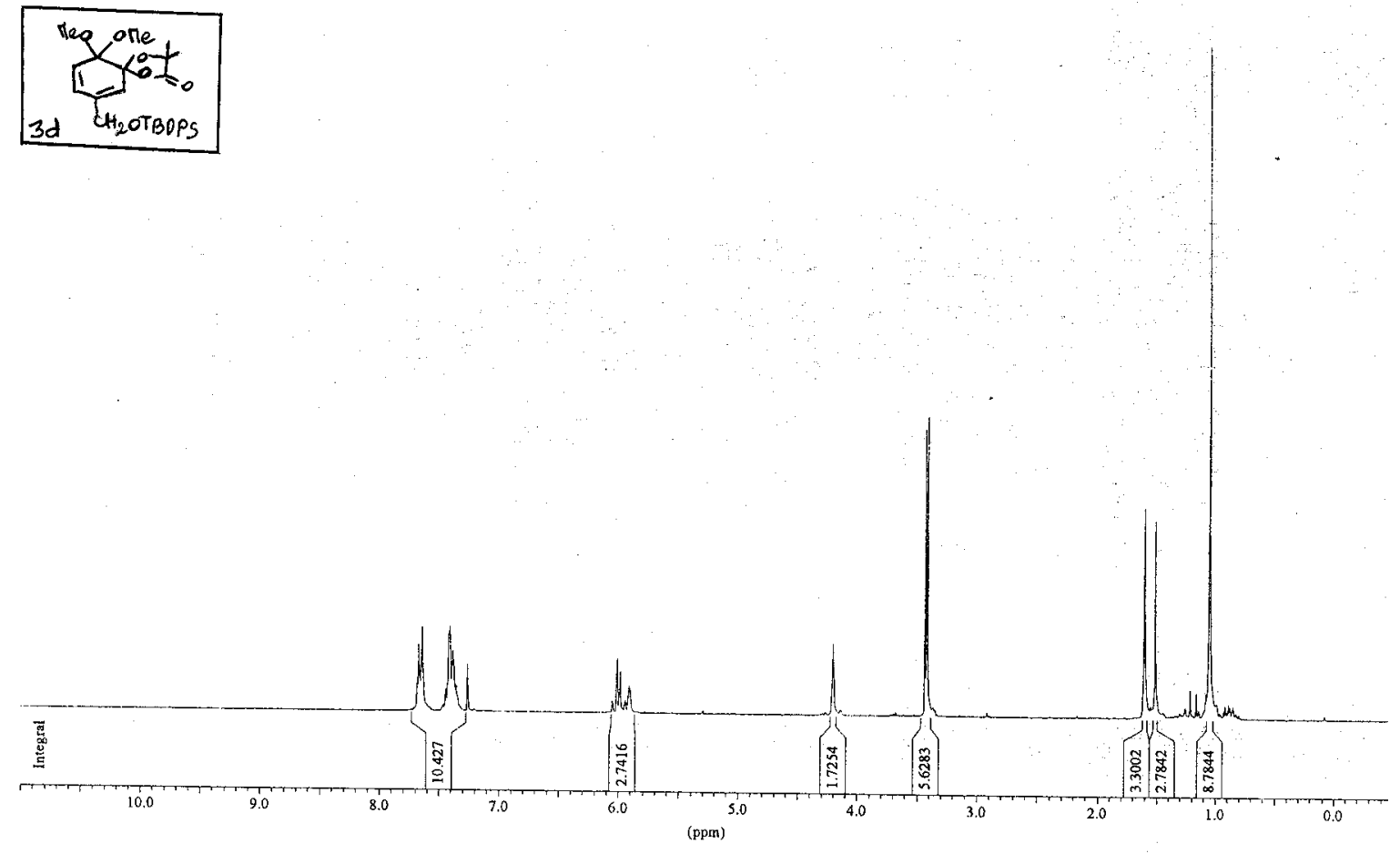


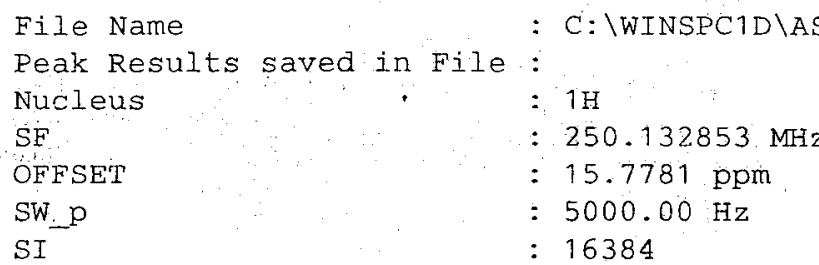

Peak Picking Parameter

Peak constant $P C=$

Noise

$$
1.00
$$

Sens. level =

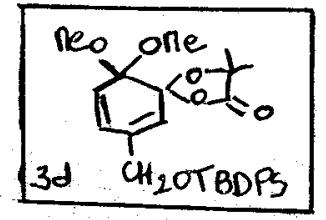

\section{Peak Picking region}

\begin{tabular}{rrrr} 
Start (ppm/Hz) & End (ppm/Hz) & MI (8) & MAXI (o) \\
\hline $7.85 / 1963.0$ & $7.55 / 1888.8$ & 4.19 & 13.96 \\
$7.48 / 1871.7$ & $7.30 / 1824.7$ & 0.65 & 14.43 \\
$7.30 / 1825.9$ & $7.05 / 1763.1$ & -0.30 & 7.99 \\
$6.15 / 1539.1$ & $5.78 / 1445.7$ & 0.52 & 9.16 \\
$4.25 / 1063.6$ & $4.03 / 1008.1$ & 8.51 & 12.36 \\
$3.61 / 902.8$ & $3.29 / 823.4$ & 36.63 & 52.71 \\
$1.71 / 428.2$ & $1.29 / 323.6$ & 26.09 & 45.45 \\
$1.13 / 283.6$ & $0.95 / 238.4$ & 90.54 & 120.62 \\
$13.82 / 3457.1$ & $12.93 / 3234.3$ & 39.62 & 42.00 \\
\hline
\end{tabular}

Peak Picking results

$\begin{array}{rrrrrr}\text { Peak Nr. Data Point } & \text { Frequency } & \text { PPM } & \text { Intensity } & \text { \% Int. } \\ 1 & 6636 & 1921.46 & 7.6818 & 676 & 4.8 \\ 2 & 6641 & 1919.94 & 7.6757 & 1363 & 9.7 \\ 3 & 6646 & 1918.41 & 7.6696 & 1222 & 8.7 \\ 4 & 6652 & 1916.58 & 7.6623 & 761 & 5.4 \\ 5 & 6660 & 1914.14 & 7.6525 & 1142 & 8.1 \\ 6 & 6666 & 1912.31 & 7.6452 & 1745 & 12.4 \\ 7 & 6672 & 1910.48 & 7.6378 & 1118 & 7.9 \\ 8 & 6804 & 1870.19 & 7.4768 & 97 & 0.7 \\ 9 & 6810 & 1868.36 & 7.4695 & 132 & 0.9 \\ 10 & 6831 & 1861.95 & 7.4439 & 461 & 3.3 \\ 11 & 6833 & 1861.34 & 7.4414 & 460 & 3.3 \\ 12 & 6844 & 1857.99 & 7.4280 & 706 & 5.0 \\ 13 & 6850 & 1856.16 & 7.4207 & 1602 & 11.4 \\ 14 & 6857 & 1854.02 & 7.4121 & 1758 & 12.5 \\ 15 & 6877 & 1847.92 & 7.3877 & 1249 & 8.9 \\ 16 & 6883 & 1846.09 & 7.3804 & 1168 & 8.3 \\ 17 & 6895 & 1842.42 & 7.3658 & 479 & 3.4 \\ 18 & 6903 & 1839.98 & 7.3560 & 511 & 3.6 \\ 19 & 6911 & 1837.54 & 7.3463 & 316 & 2.2 \\ 20 & 6950 & 1825.64 & 7.2987 & 20 & 0.1\end{array}$




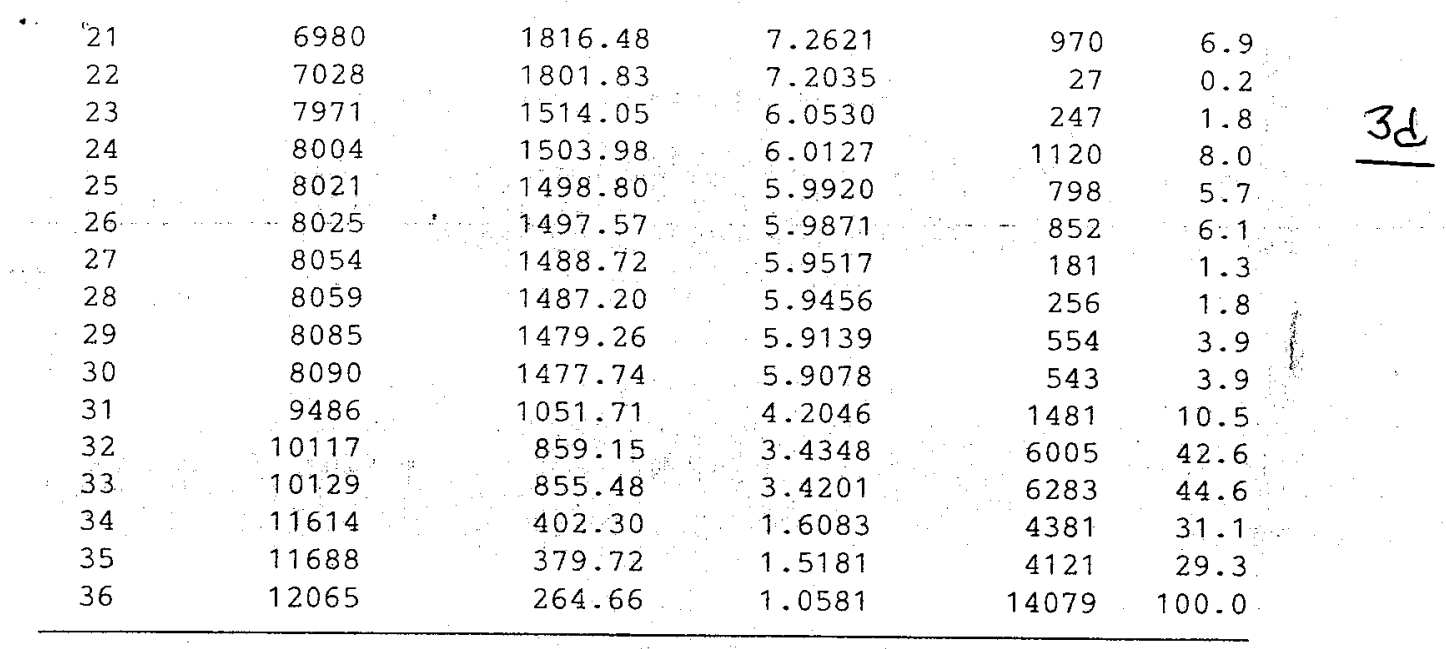




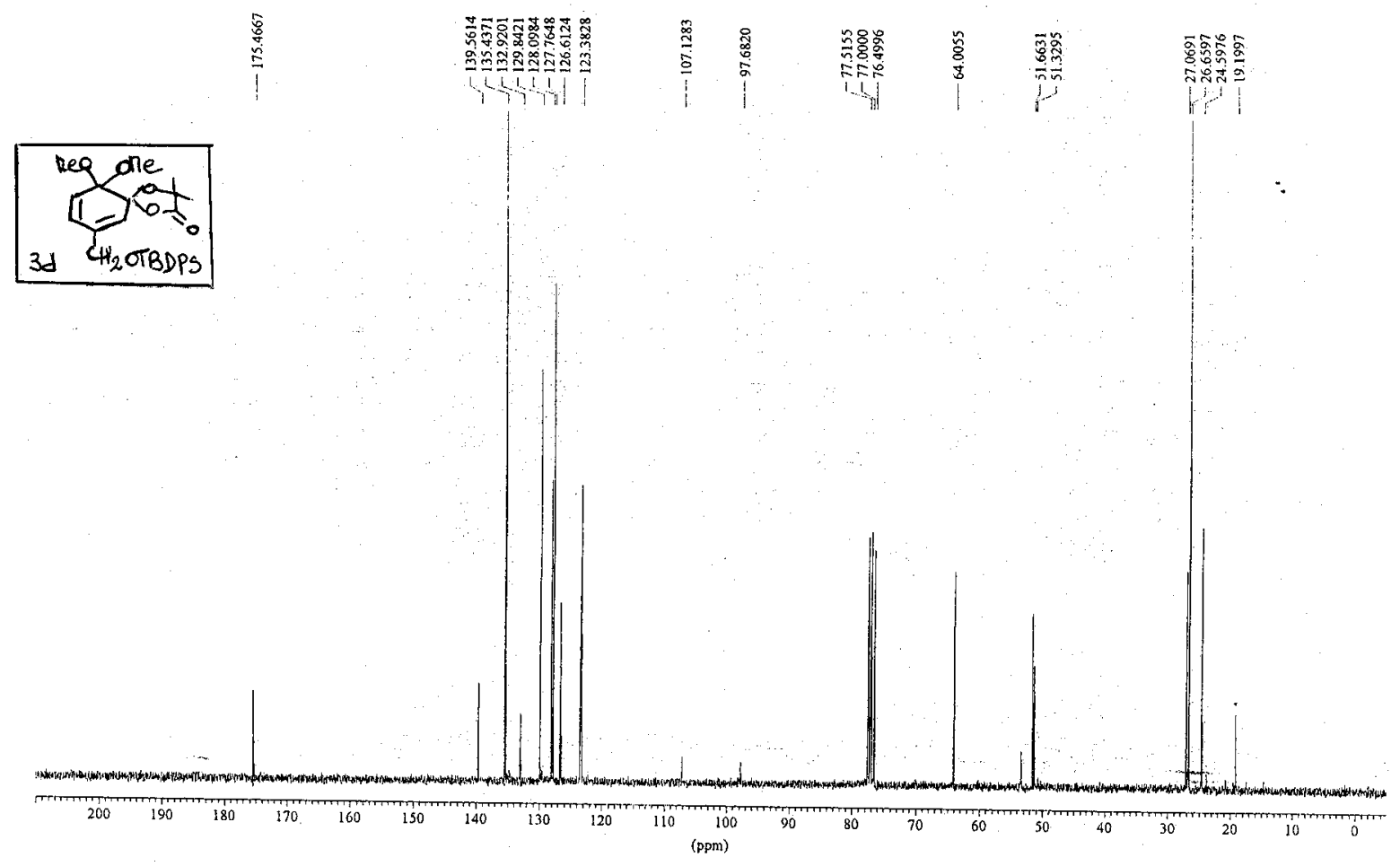

IFA153/CDC13/FV28-101/AC 250
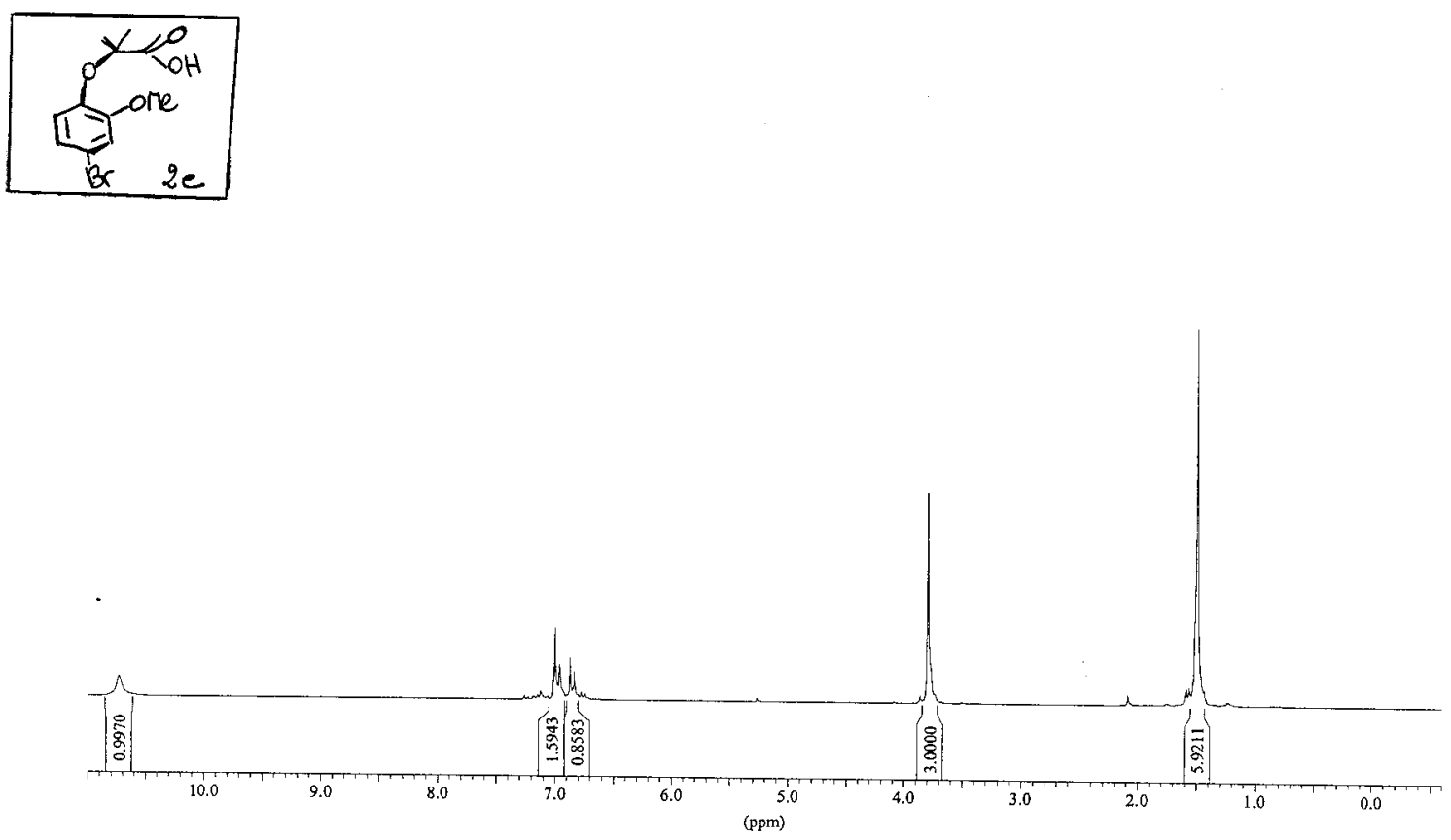
File Name

: C: \WINSPC1D \ASP3000\FV280F\101001.1R

Peak Results saved in File:

Nucleus

$\mathrm{SF}$

$: 1 \mathrm{H}$

OFFSET

$S W \_P$

$: 250.132855 \mathrm{MHz}$

: 15.7723 ppm

: $5000.00 \mathrm{~Hz}$

$S I$

: 16384

Peak Picking Parameter

$\begin{array}{lrr}\text { Peak constant PC } & = & 1.00 \\ \text { Noise level } & = & 1 \\ \text { Sens. level } & & 6\end{array}$

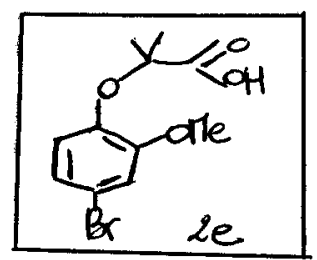

Peak Picking region

\begin{tabular}{|c|c|c|c|}
\hline Start $(\mathrm{ppm} / \mathrm{Hz})$ & End $(\mathrm{ppm} / \mathrm{Hz})$ & $\operatorname{MI}\left(\frac{8}{0}\right)$ & $\operatorname{MAXI}(8)$ \\
\hline $6.89 / 1724.1$ & $6.79 / 1698.2$ & 6.08 & 12.91 \\
\hline $6.99 / 1747.6$ & $6.91 / 1729.0$ & 7.44 & 9.65 \\
\hline $7.00 / 1751.6$ & $6.99 / 1748.2$ & 17.33 & 19.89 \\
\hline $7.28 / 1820.5$ & $7.24 / 1810.2$ & 0.51 & 3.12 \\
\hline $10.79 / 2699.4$ & $10.70 / 2677.2$ & 2.82 & 5.28 \\
\hline $6.79 / 1699.1$ & $6.70 / 1676.8$ & 1.39 & 2.62 \\
\hline $3.81 / 954.1$ & $3.68 / 920.6$ & 56.55 & 67.19 \\
\hline $1.54 /$ & 368.5 & 96.26 & 108.95 \\
\hline
\end{tabular}

Peak Picking results

\begin{tabular}{rrrrrr} 
Peak Nr. Data Point & Frequency & \multicolumn{1}{c}{ PPM } & Intensity & 8Int. \\
1 & 4133 & 2683.88 & 10.7298 & 472 & 3.5 \\
2 & 6978 & 1815.66 & 7.2588 & 165 & 1.2 \\
3 & 7194 & 1749.74 & 6.9952 & 2544 & 18.7 \\
4 & 7221 & 1741.50 & 6.9623 & 1205 & 8.8 \\
5 & 7228 & 1739.37 & 6.9538 & 1027 & 7.5 \\
6 & 7298 & 1718.00 & 6.8684 & 1532 & 11.3 \\
7 & 7324 & 1710.07 & 6.8366 & 920 & 6.8 \\
8 & 7375 & 1694.50 & 6.7744 & 238 & 1.7 \\
9 & 7403 & 1685.96 & 6.7403 & 198 & 1.5 \\
10 & 9815 & 949.88 & 3.7975 & 8434 & 61.9 \\
11 & 11702 & 374.01 & 1.4952 & 13621 & 100.0 \\
\hline
\end{tabular}


IFA153/CC13/FV28-101/AC 250

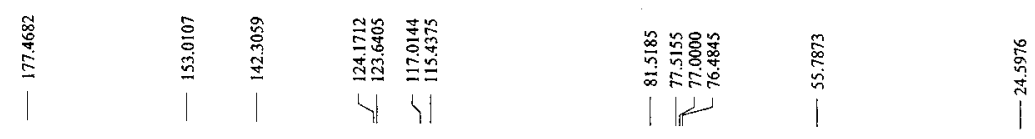
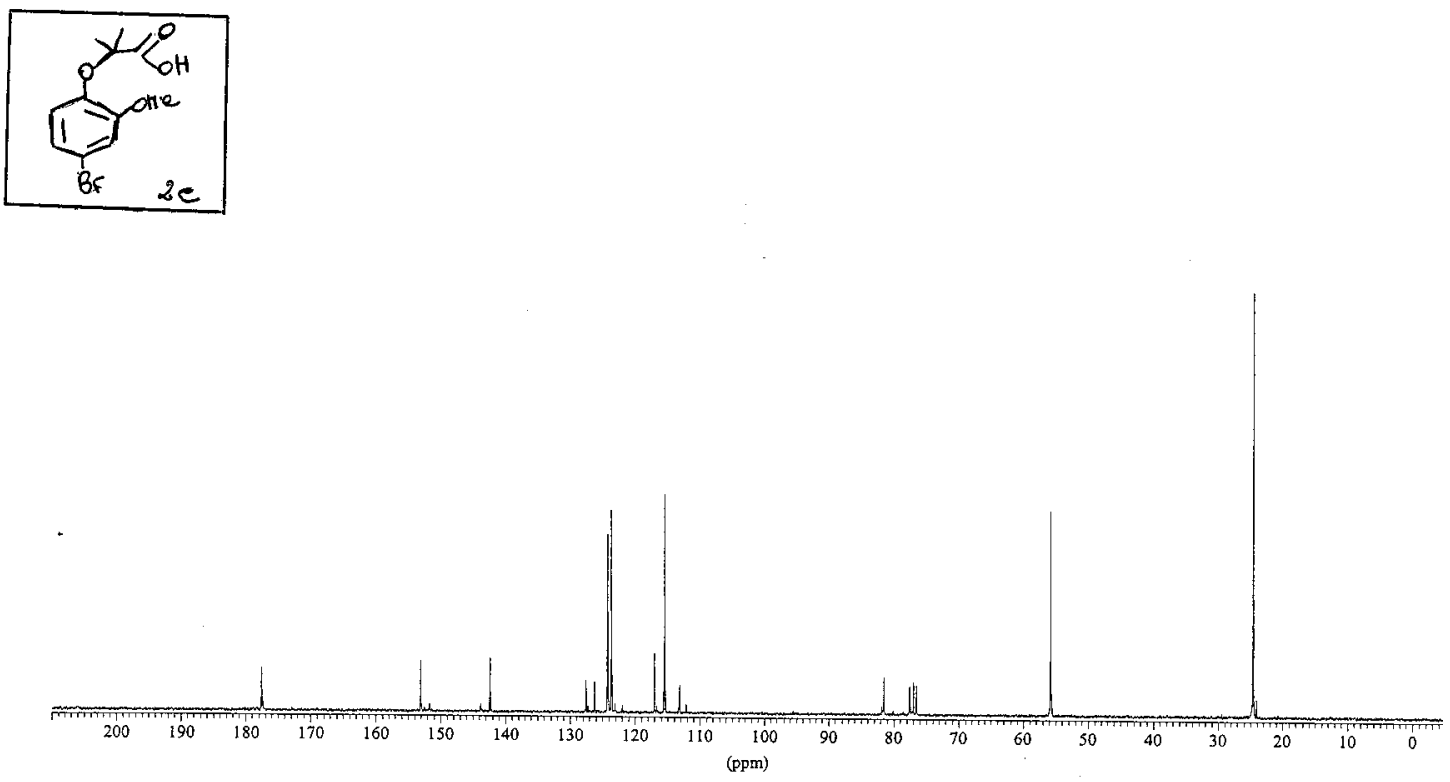

IFAI58/CDCI3/FV28-102/AC 250
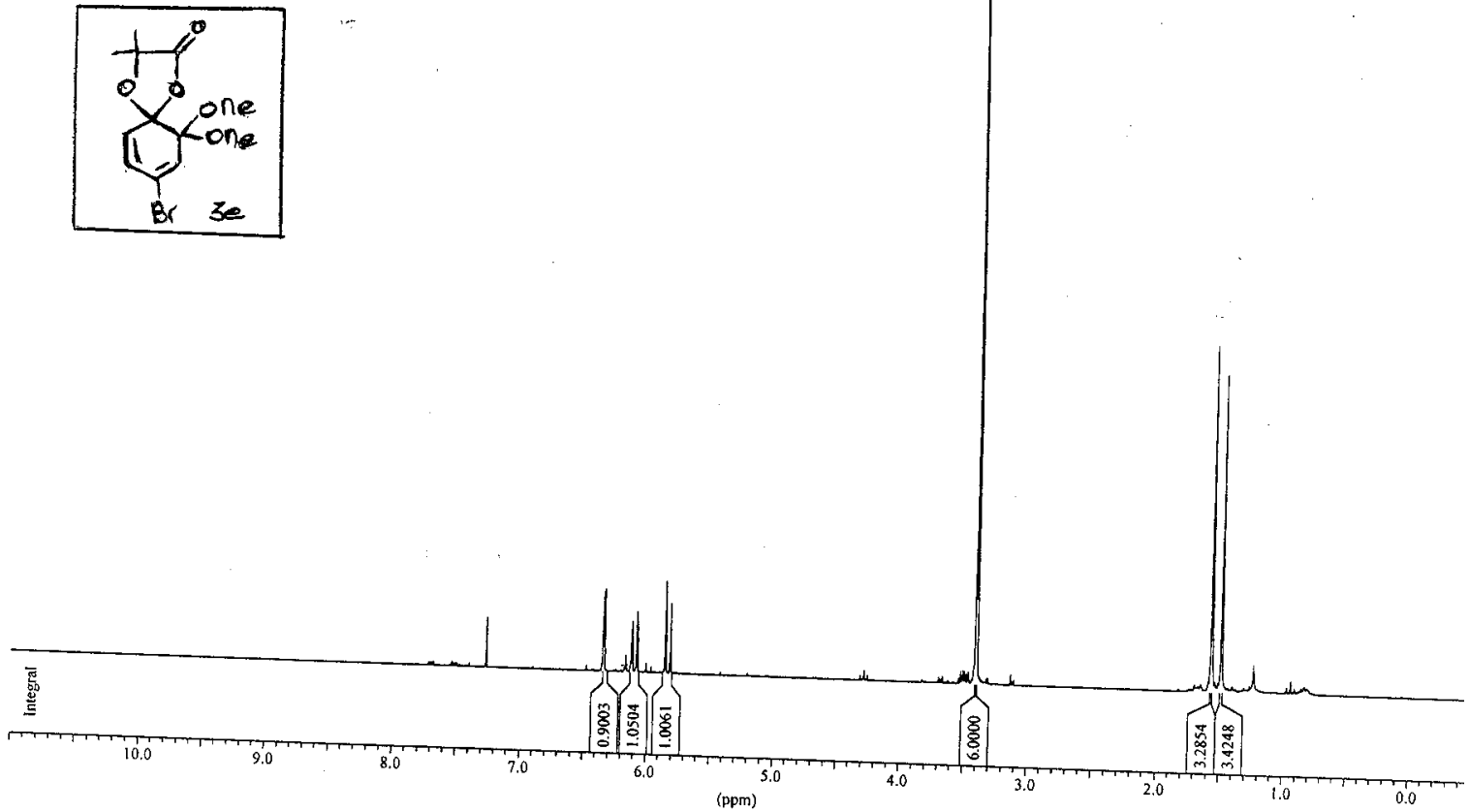


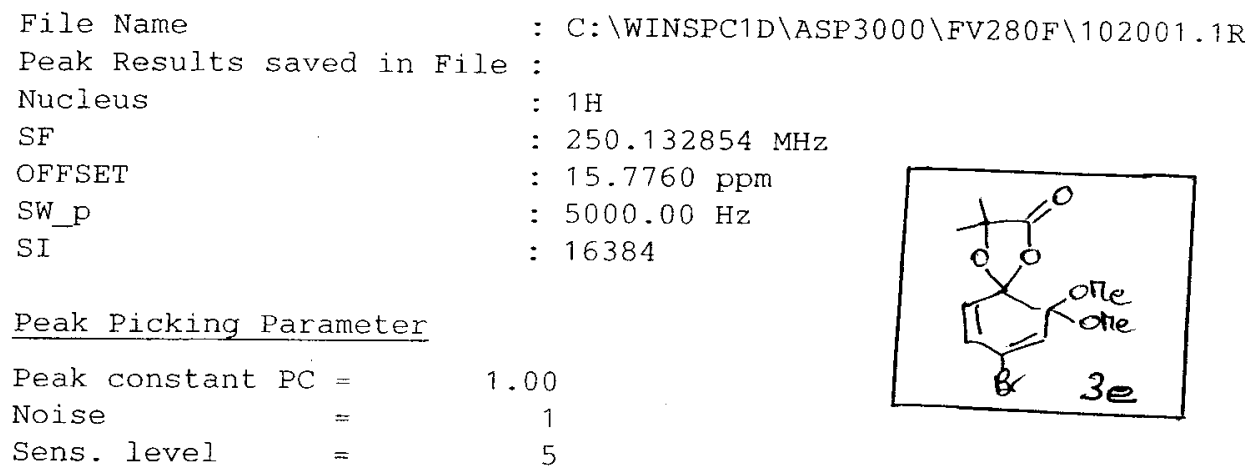

$\mathrm{SI}^{-} \quad 16384$

Peak Picking Parameter

Peak constant $\mathrm{PC}=$ 1.00

Noise

Sens. level =

\section{Peak Picking region}

\begin{tabular}{llrr} 
Start $(\mathrm{ppm} / \mathrm{Hz})$ & End $(\mathrm{ppm} / \mathrm{Hz})$ & MI $(\%)$ & \multicolumn{1}{c}{ MAXI (\%) } \\
\hline $5.89 / 1472.6$ & $5.81 / 1452.5$ & 10.60 & 11.54 \\
$5.83 / 1458.0$ & $5.78 / 1446.4$ & 7.37 & 9.30 \\
$6.09 / 1523.9$ & $6.05 / 1514.1$ & 5.55 & 7.74 \\
$6.14 / 1535.5$ & $6.09 / 1523.3$ & 5.21 & 6.71 \\
$6.36 / 1591.1$ & $6.31 / 1579.5$ & 8.56 & 10.41 \\
$7.30 / 1826.3$ & $7.21 / 1802.8$ & 4.58 & 7.14 \\
$1.93 / 481.7$ & $1.27 / 318.5$ & 36.65 & 45.91 \\
$3.76 / 941.6$ & $1.75 / 437.2$ & 27.75 & 113.64 \\
\hline
\end{tabular}

Peak Picking results

\begin{tabular}{rrrrrr} 
Peak Nr. Data Point & Frequency & PPM & Intensity & o Int. \\
\hline 1 & 6980 & 1815.96 & 7.2600 & 1200 & 5.9 \\
2 & 7735 & 1585.56 & 6.3389 & 1832 & 9.0 \\
3 & 7741 & 1583.73 & 6.3315 & 1982 & 9.8 \\
4 & 7917 & 1530.01 & 6.1168 & 1120 & 5.5 \\
5 & 7923 & 1528.18 & 6.1095 & 1235 & 6.1 \\
6 & 7949 & 1520.25 & 6.0778 & 1469 & 7.3 \\
7 & 7956 & 1518.11 & 6.0692 & 1126 & 5.6 \\
8 & 8137 & 1462.88 & 5.8484 & 2249 & 11.1 \\
9 & 8170 & 1452.81 & 5.8081 & 1708 & 8.4 \\
10 & 10139 & 851.91 & 3.4058 & 20245 & 100.0 \\
11 & 10146 & 849.78 & 3.3973 & 17234 & 85.1 \\
12 & 11649 & 391.10 & 1.5636 & 8467 & 41.8 \\
13 & 11715 & 370.96 & 1.4830 & 7732 & 38.2 \\
\hline
\end{tabular}


IFA158-1/CDC13/FV28-102/AC 250
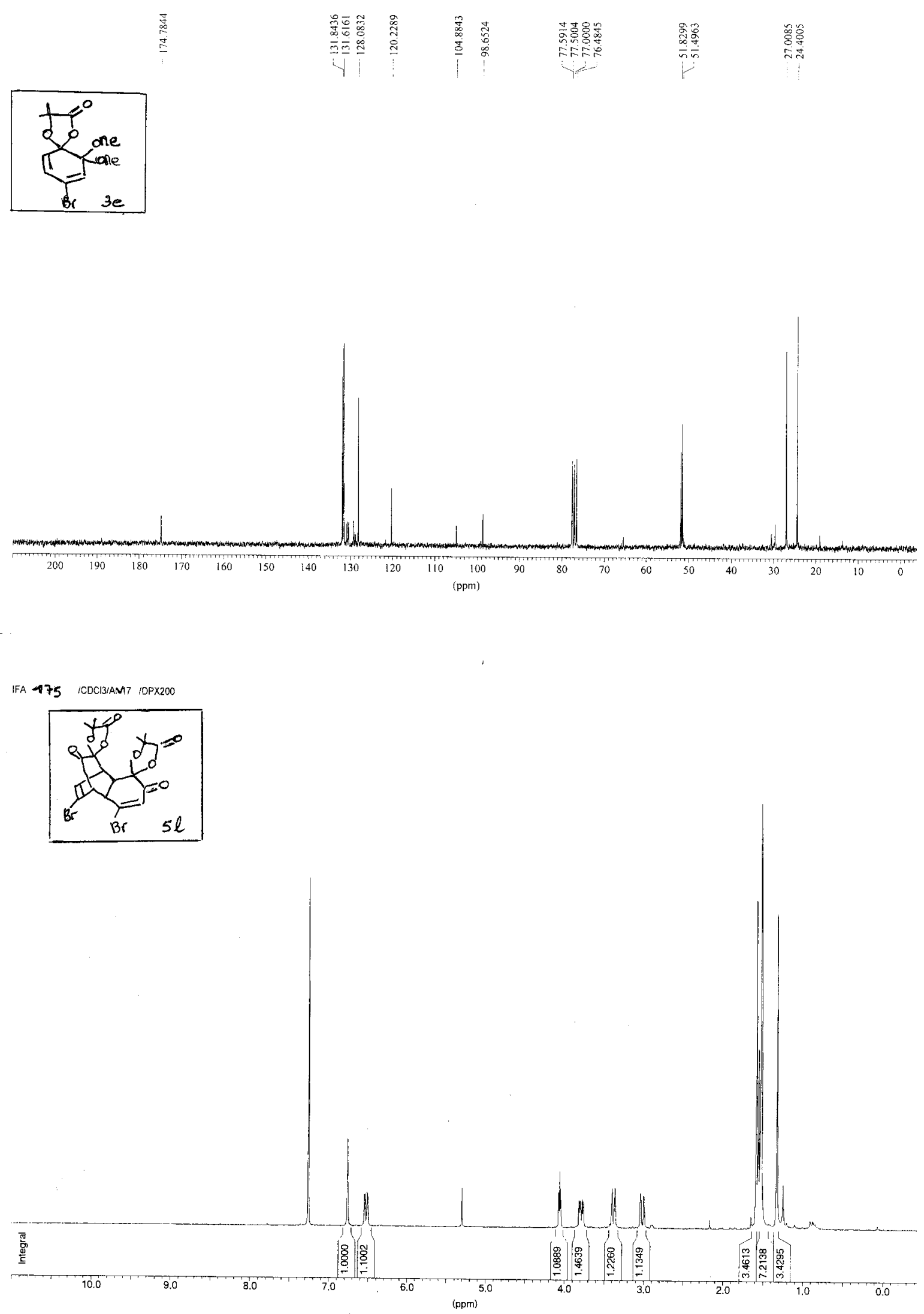
File Name

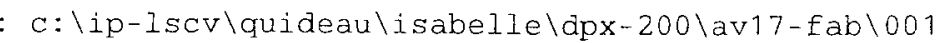

Peak Results saved in File:

Nucleus

$\mathrm{SF}$

: off

OFFSET

$200.160007 \mathrm{MHz}$

SW_p

: $11.9682 \mathrm{ppm}$

: $2604.17 \mathrm{~Hz}$

$S I$

: 16384

Peak Picking Parameter

Peak constant $\mathrm{PC}=1.00$

Noise $=291095$

Sens. level = 1164378

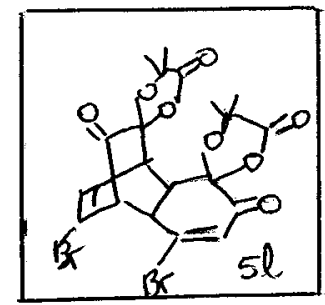

Peak Picking region

\begin{tabular}{llrl} 
Start $(\mathrm{ppm} / \mathrm{Hz})$ & End $(\mathrm{ppm} / \mathrm{Hz})$ & MI (\%) & MAXI (\%) \\
\hline $8.09 / 1619.3$ & $6.61 / 1323.5$ & 71.29 & 92.38 \\
$6.80 / 1360.2$ & $6.74 / 1350.0$ & 19.42 & 23.03 \\
$6.78 / 1357.5$ & $6.76 / 1353.7$ & 19.03 & 22.18 \\
$6.78 / 1357.9$ & $6.76 / 1353.7$ & 19.31 & 22.07 \\
$6.77 / 1354.6$ & $6.75 / 1350.3$ & 18.74 & 22.80 \\
$6.56 / 1313.6$ & $6.48 / 1296.4$ & 5.39 & 11.42 \\
$5.33 / 1066.4$ & $5.25 / 1051.2$ & 5.27 & 15.59 \\
$4.10 / 220.4$ & $3.99 / 798.9$ & 4.09 & 19.48 \\
$1.37 / 273.5$ & $1.29 / 257.6$ & 47.47 & 80.84 \\
\hline
\end{tabular}

Peak Picking results

\begin{tabular}{rrrrrr} 
Peak Nr. Data Point & Frequency & PPM & Intensity & \% Int. \\
\hline 1 & 5929 & 1453.16 & 7.2600 & 222523824 & 81.7 \\
2 & 6557 & 1353.34 & 6.7613 & 55931504 & 20.5 \\
3 & 6825 & 1310.75 & 6.5485 & 20867370 & 7.7 \\
4 & 6838 & 1308.68 & 6.5382 & 20951148 & 7.7 \\
5 & 6869 & 1303.75 & 6.5136 & 22012624 & 8.1 \\
6 & 6882 & 1301.69 & 6.5032 & 21223716 & 7.8 \\
7 & 8398 & 1060.72 & 5.2994 & 25056710 & 9.2 \\
8 & 9941 & 815.47 & 4.0741 & 22861218 & 8.4 \\
9 & 9957 & 812.93 & 4.0614 & 36353172 & 13.4 \\
10 & 9972 & 810.54 & 4.0495 & 25844076 & 9.5 \\
11 & 10267 & 763.66 & 3.8152 & 17775684 & 6.5 \\
12 & 10286 & 760.64 & 3.8001 & 17876236 & 6.6 \\
13 & 10320 & 755.23 & 3.7731 & 18555990 & 6.8 \\
14 & 10335 & 752.85 & 3.7612 & 17082474 & 6.3 \\
15 & 10790 & 680.53 & 3.3999 & 25032894 & 9.2 \\
16 & 10799 & 679.10 & 3.3928 & 26290194 & 9.7 \\
17 & 10834 & 673.53 & 3.3650 & 25139050 & 9.2 \\
18 & 10844 & 671.94 & 3.3570 & 26166092 & 9.6 \\
19 & 11239 & 609.16 & 3.0434 & 22843324 & 8.4 \\
20 & 11248 & 607.73 & 3.0362 & 22923000 & 8.4
\end{tabular}




\begin{tabular}{rrrrrrr}
21 & 11292 & 600.74 & 3.0013 & 21223206 & 7.8 & 5? \\
22 & 11301 & 599.31 & 2.9941 & 20896142 & 7.7 & \\
23 & 13073 & 317.65 & 1.5870 & 210615568 & 77.4 & \\
24 & 13107 & 312.25 & 1.5600 & 115934720 & 42.6 \\
25 & 13142 & 306.69 & 1.5322 & 251104560 & 92.2 & \\
26 & 13150 & 305.41 & 1.5259 & 272242592 & 100.0 & \\
27 & 13400 & 265.68 & 1.3273 & 201980688 & 74.2 \\
\hline
\end{tabular}

IFA175/CDCL3/AV17/OPX 200
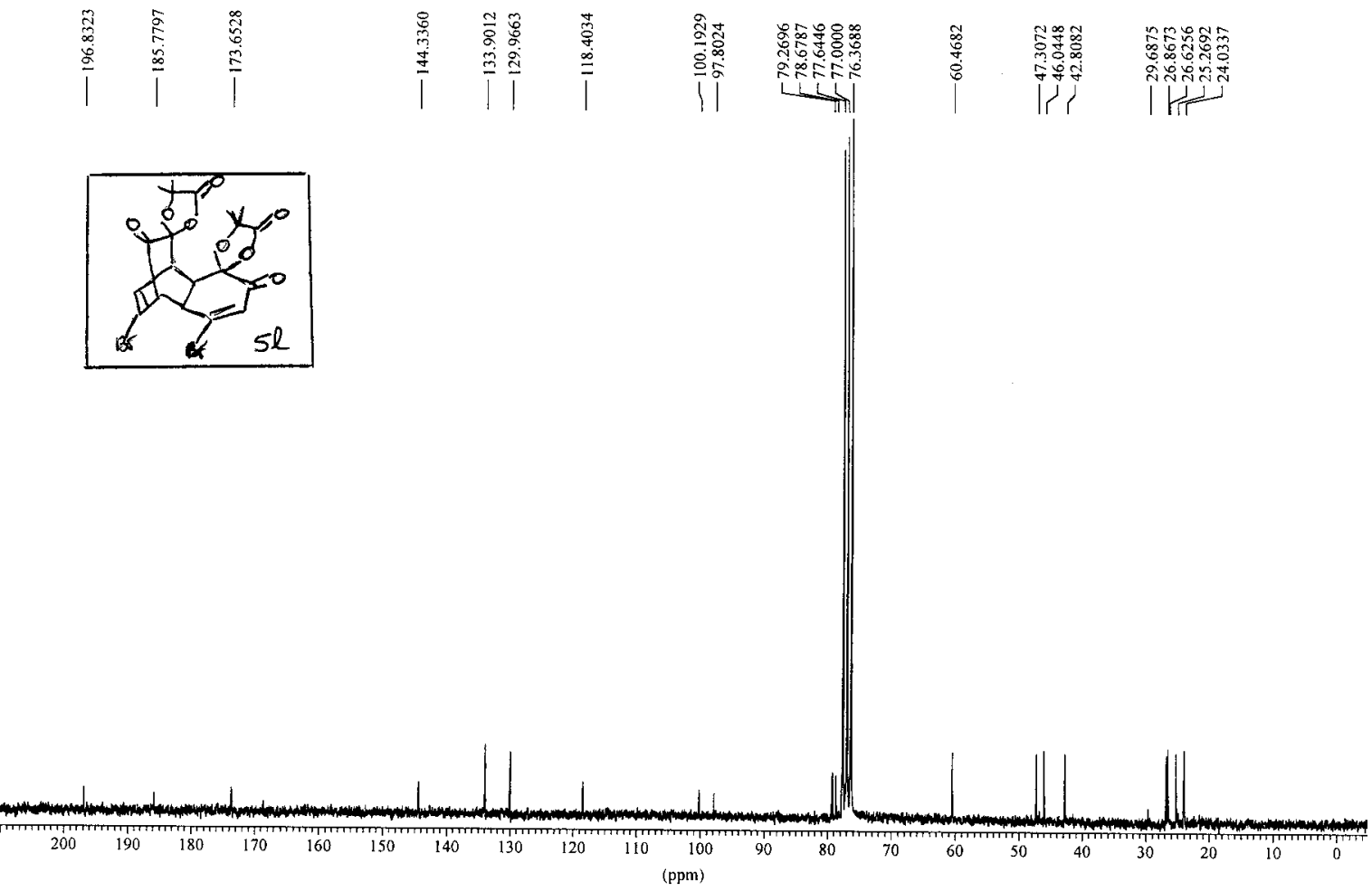
IFB 117.2/CDCl3/M107-101/AC 250
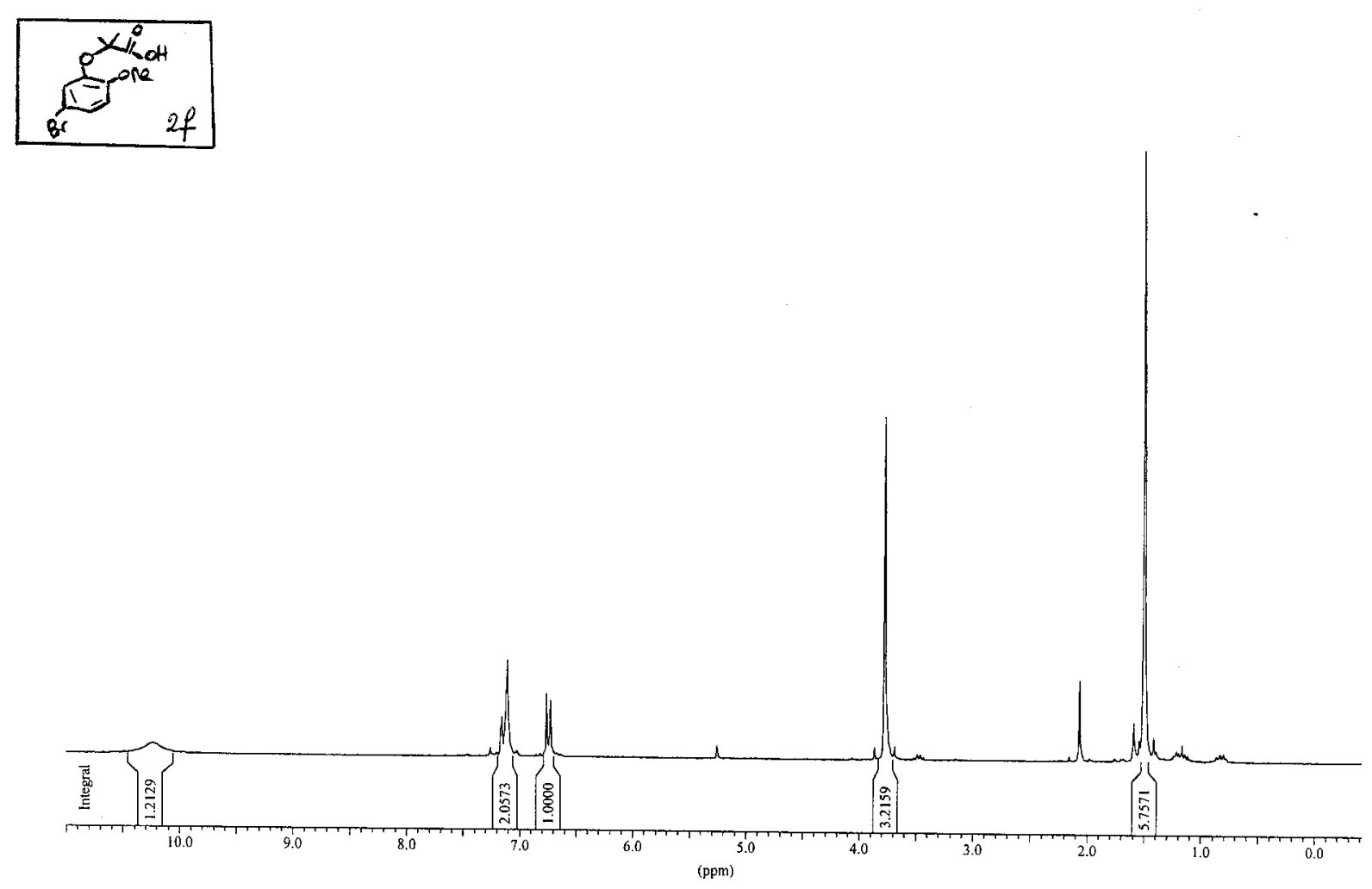

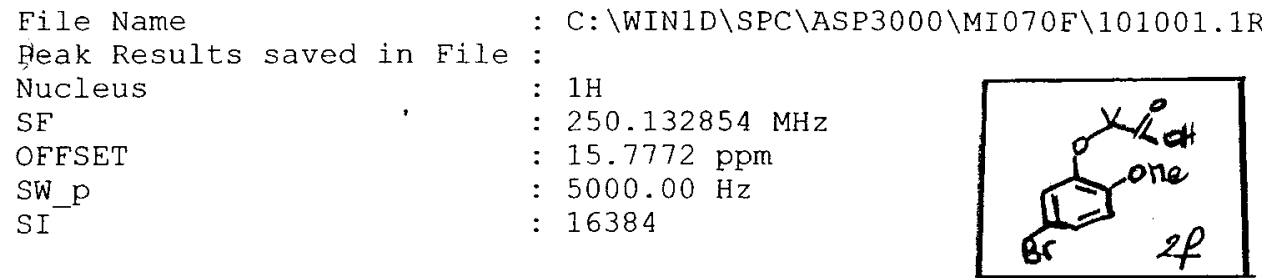

Peak Picking Parameter

Peak constant $\mathrm{PC}=$ Noise

$=1.00$

Sens. level =

.00

1

Peak Picking region

\begin{tabular}{rrrr} 
Start (ppm/Hz) & End (ppm/Hz) & MI (\%) & \multicolumn{1}{c}{ MAXI (\%) } \\
\hline $10.63 / 2660.1$ & $9.77 / 2443.1$ & -0.02 & 4.61 \\
$7.30 / 1825.1$ & $6.30 / 1577.0$ & 0.85 & 20.90 \\
$4.64 / 1160.4$ & $3.27 / 817.4$ & 42.82 & 70.81 \\
$2.14 / 535.5$ & $0.93 / 233.6$ & 78.81 & 138.21 \\
\hline
\end{tabular}

Peak Picking results

\begin{tabular}{crrrrr} 
Peak Nr. Data Point & Erequency & PPM & Intensity & oInt. \\
\hline 1 & 4540 & 2560.90 & 10.2382 & 90 & 1.7 \\
2 & 6981 & 1815.96 & 7.2600 & 69 & 1.3 \\
3 & 7064 & 1790.63 & 7.1587 & 336 & 6.4 \\
4 & 7093 & 1781.78 & 7.1234 & 528 & 10.0 \\
5 & 7102 & 1779.04 & 7.1124 & 826 & 15.7 \\
6 & 7388 & 1691.76 & 6.7634 & 537 & 10.2 \\
7 & 7416 & 1683.21 & 6.7293 & 478 & 9.1 \\
8 & 9836 & 944.69 & 3.7767 & 2955 & 56.1 \\
9 & 11698 & 376.45 & 1.5050 & 5265 & 100.0
\end{tabular}


JFB117.2/CDCl3/M107-101/C13/AC 250
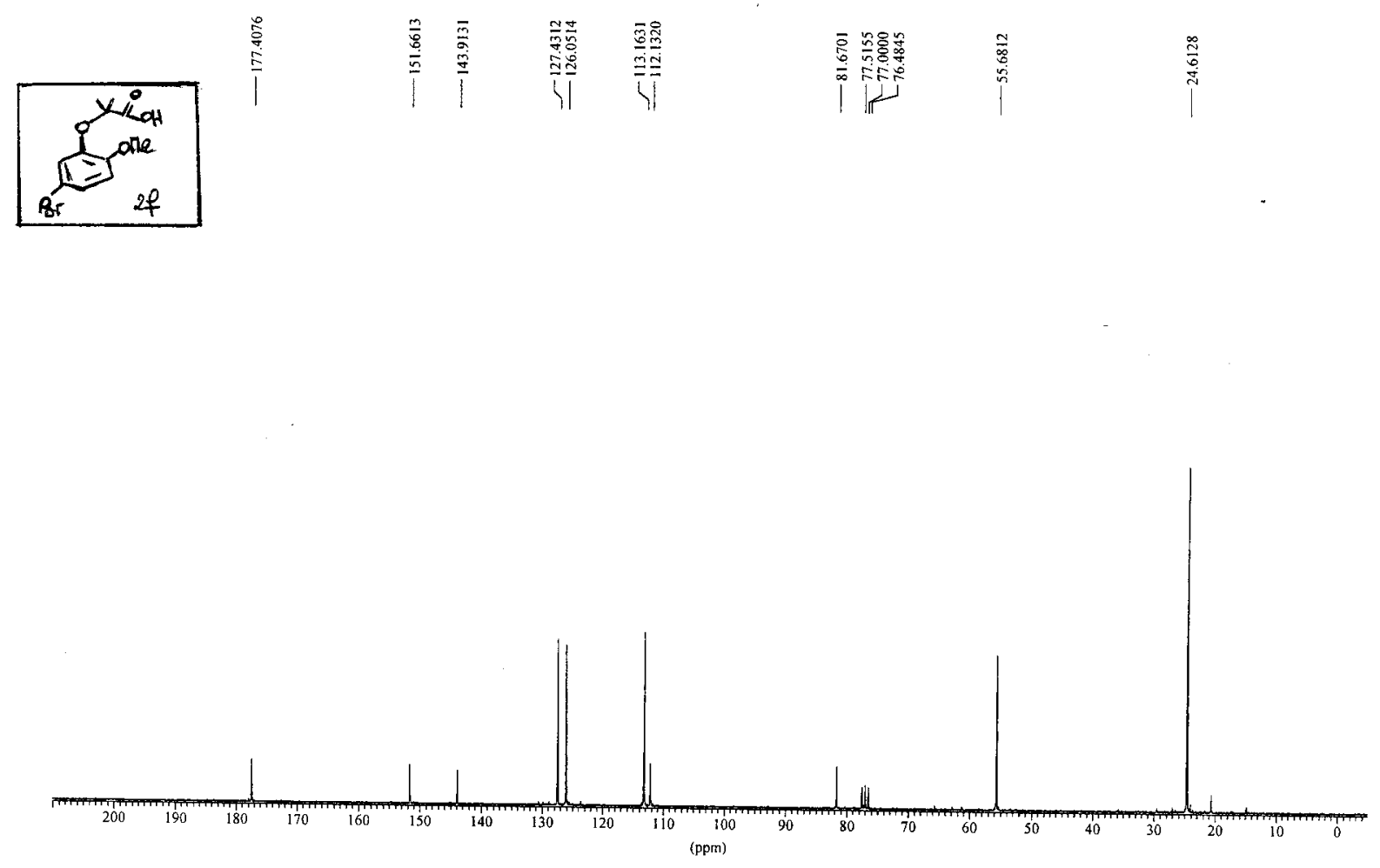

IFBI21-|/CDCI3/MA|4-iff 121-| OPXX 200
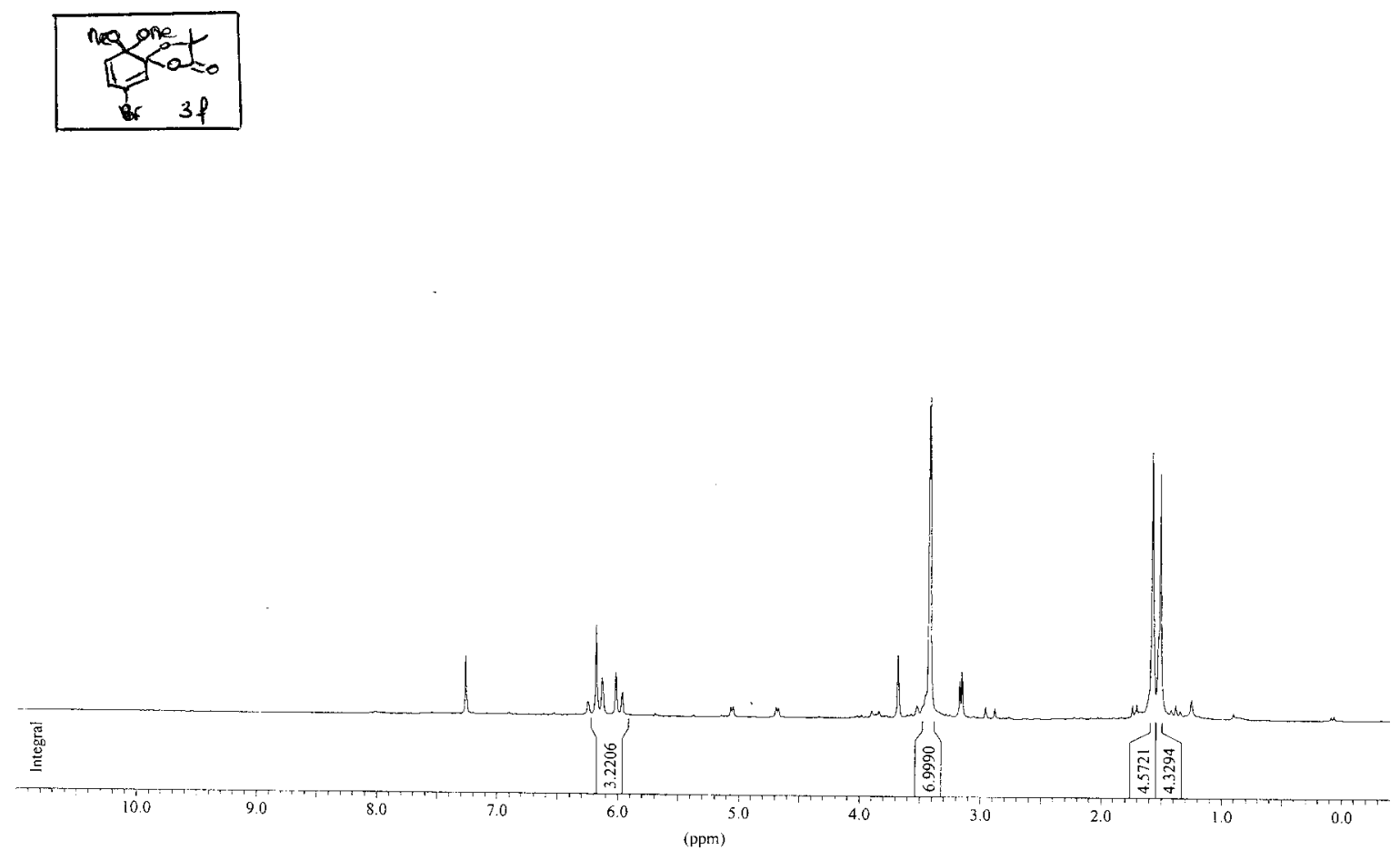
File Name

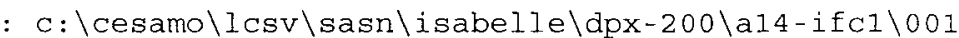

Peak Results saved in File:

Nucleus

$\mathrm{SF}$

: off

OFFSET

SW_p

: $200.160019 \mathrm{MHz}$

: $11.9086 \mathrm{ppm}$

$S I$

: $2604.17 \mathrm{~Hz}$

: 16384

Peak Picking Parameter

Peak constant $\mathrm{PC}=$

1.00

Noise

$=$
$=$

0

Sens. level

0

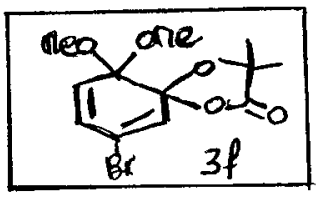

Peak Picking region

\begin{tabular}{cccc} 
Start $(\mathrm{ppm} / \mathrm{Hz})$ & End $(\mathrm{ppm} / \mathrm{Hz})$ & MI $(\%)$ & MAXI (\%) \\
\hline $11.91 / 2383.6$ & $-1.10 /-220.5$ & -0.42 & 100.00 \\
\hline
\end{tabular}

Peak Picking results

\begin{tabular}{crrrrr} 
Peak Nr. Data Point & Frequency & PPM & Intensity & \%Int. \\
\hline 1 & 5854 & 1453.16 & 7.2600 & 221152 & 18.1 \\
2 & 7214 & 1237.00 & 6.1800 & 346820 & 28.4 \\
3 & 7276 & 1227.14 & 6.1308 & 143508 & 11.7 \\
4 & 7286 & 1225.55 & 6.1229 & 123641 & 10.1 \\
5 & 7420 & 1204.25 & 6.0164 & 166358 & 13.6 \\
6 & 7487 & 1193.60 & 5.9632 & 91608 & 7.5 \\
7 & 10691 & 684.34 & 3.4190 & 1190229 & 97.4 \\
8 & 10703 & 682.43 & 3.4094 & 1222064 & 100.0 \\
9 & 13014 & 315.11 & 1.5743 & 1017410 & 83.3 \\
10 & 13100 & 301.44 & 1.5060 & 936768 & 76.7 \\
\hline
\end{tabular}


iFB121-1/CDCL3/MA14-ifb 121-1//PPX200
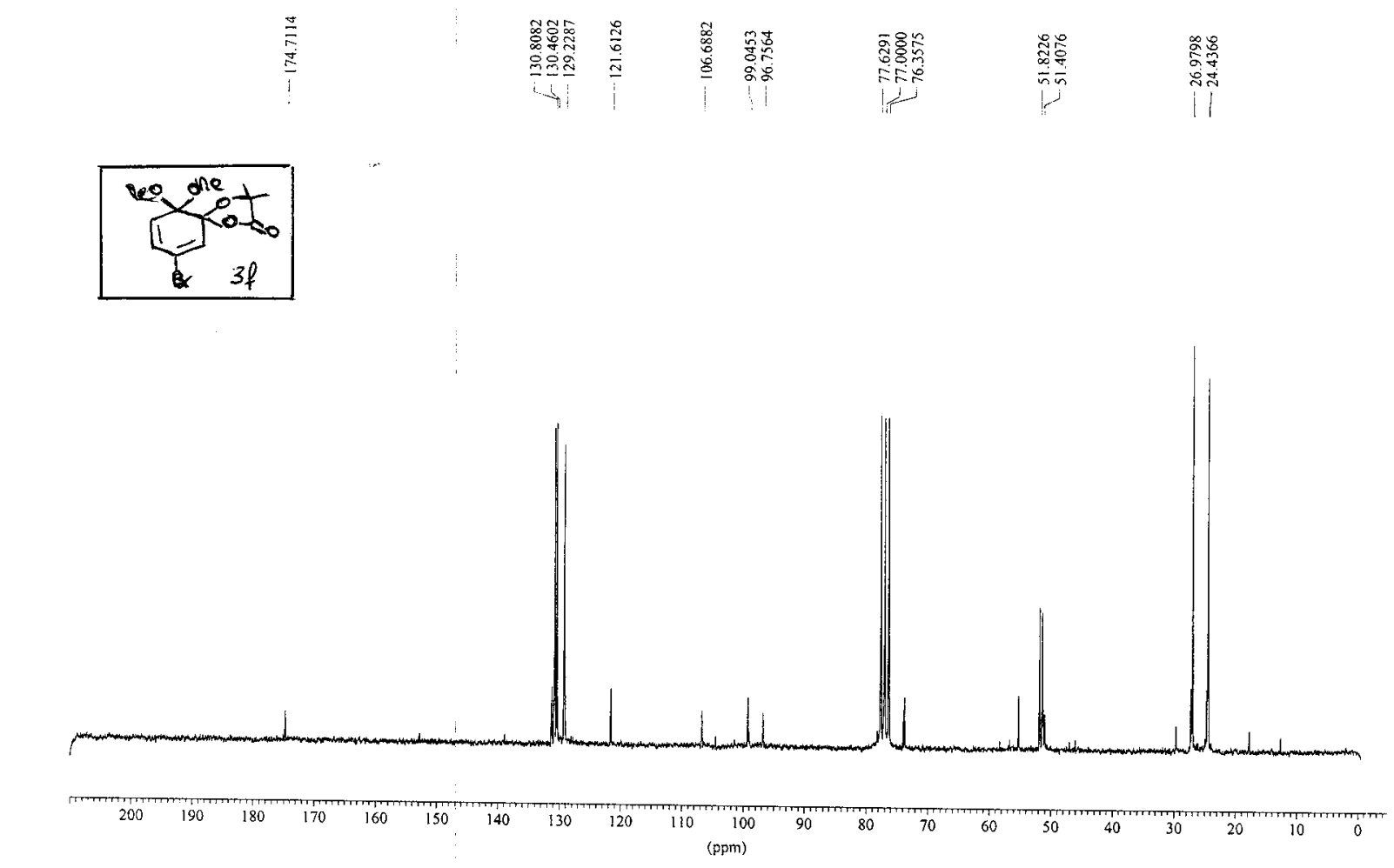

IFB94-1/CDCI3/MR05-fabre94-i,DPX 200

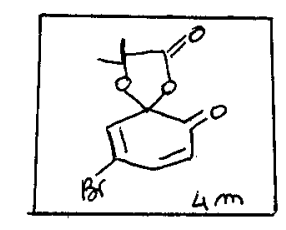

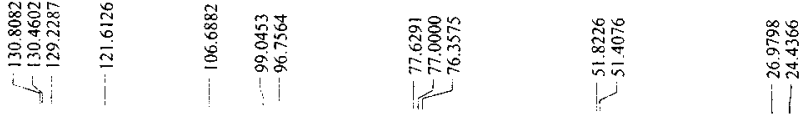

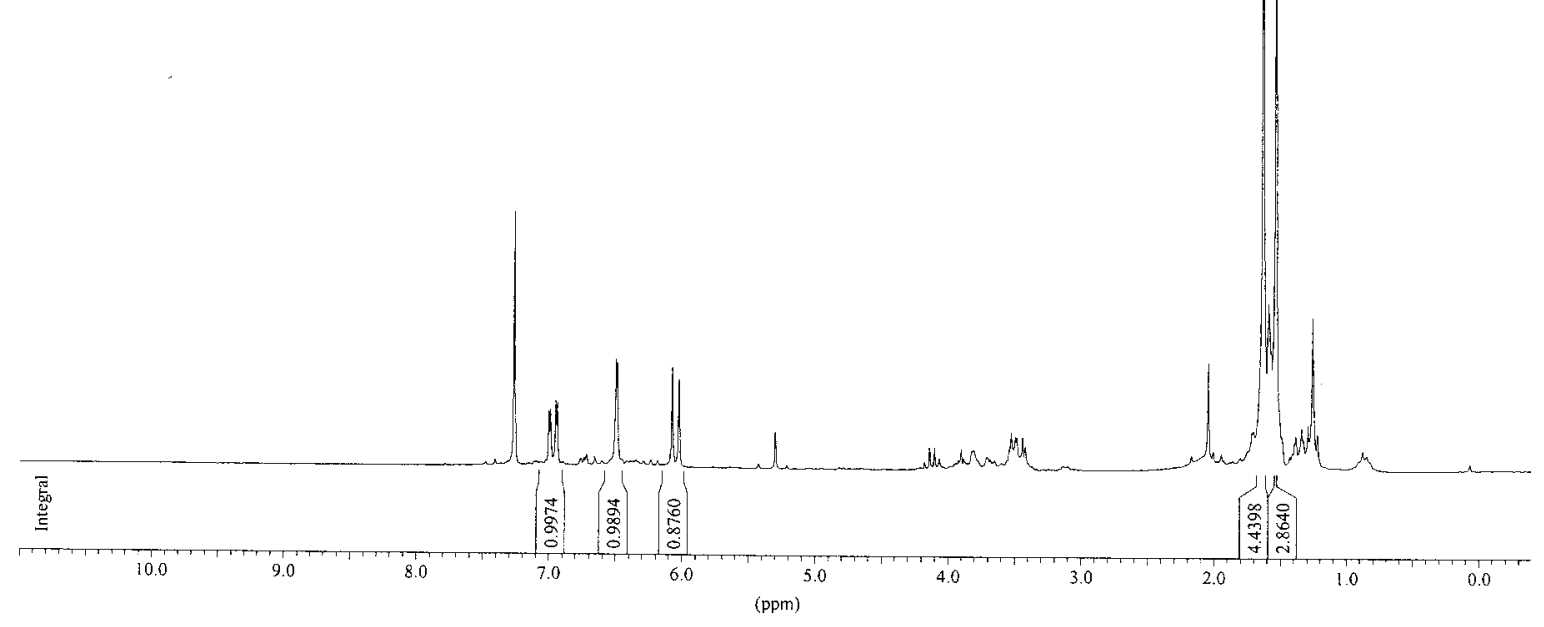


File Name b: \2002-2 I Idpx200 1 $\backslash 05-$ fabre $001001.1 \mathrm{R}$

Peak Results saved in File: Nucleus

$S F$ : Off

OFFSET

$S W$

: $200.160007 \mathrm{MHz}$

: $11.9682 \mathrm{ppm}$

: $2604.17 \mathrm{~Hz}$

: 16384

Peak Picking Parameter

Peak constant $\mathrm{PC}=$

1.00

Noise

$=$
$=$

0

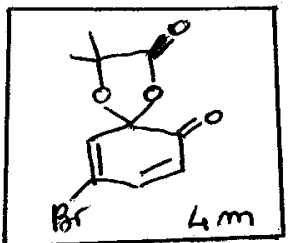

Peak Picking region

\begin{tabular}{cccc} 
Start $(\mathrm{ppm} / \mathrm{Hz})$ & End $(\mathrm{ppm} / \mathrm{Hz})$ & MI $(\%)$ & MAXI $(\%)$ \\
\hline $11.97 / 2395.6$ & $-1.04 /-208.6$ & -0.47 & 100.00 \\
\hline
\end{tabular}

Peak Picking results

\begin{tabular}{rrrrrr} 
Peak Nr. Data Point & Frequency & PPM & Intensity & $\%$ Int. \\
\hline 1 & 5929 & 1453.16 & 7.2600 & 35667 & 36.1 \\
2 & 6258 & 1400.87 & 6.9987 & 7862 & 7.9 \\
3 & 6272 & 1398.64 & 6.9876 & 8126 & 8.2 \\
4 & 6322 & 1390.70 & 6.9479 & 9265 & 9.4 \\
5 & 6337 & 1388.31 & 6.9360 & 9053 & 9.2 \\
6 & 6891 & 1300.26 & 6.4961 & 15192 & 15.4 \\
7 & 6905 & 1298.03 & 6.4850 & 14677 & 14.8 \\
8 & 7424 & 1215.54 & 6.0728 & 14117 & 14.3 \\
9 & 7488 & 1205.37 & 6.0220 & 12478 & 12.6 \\
10 & 13023 & 325.60 & 1.6267 & 98909 & 100.0 \\
11 & 13142 & 306.69 & 1.5322 & 96846 & 97.9 \\
\hline
\end{tabular}




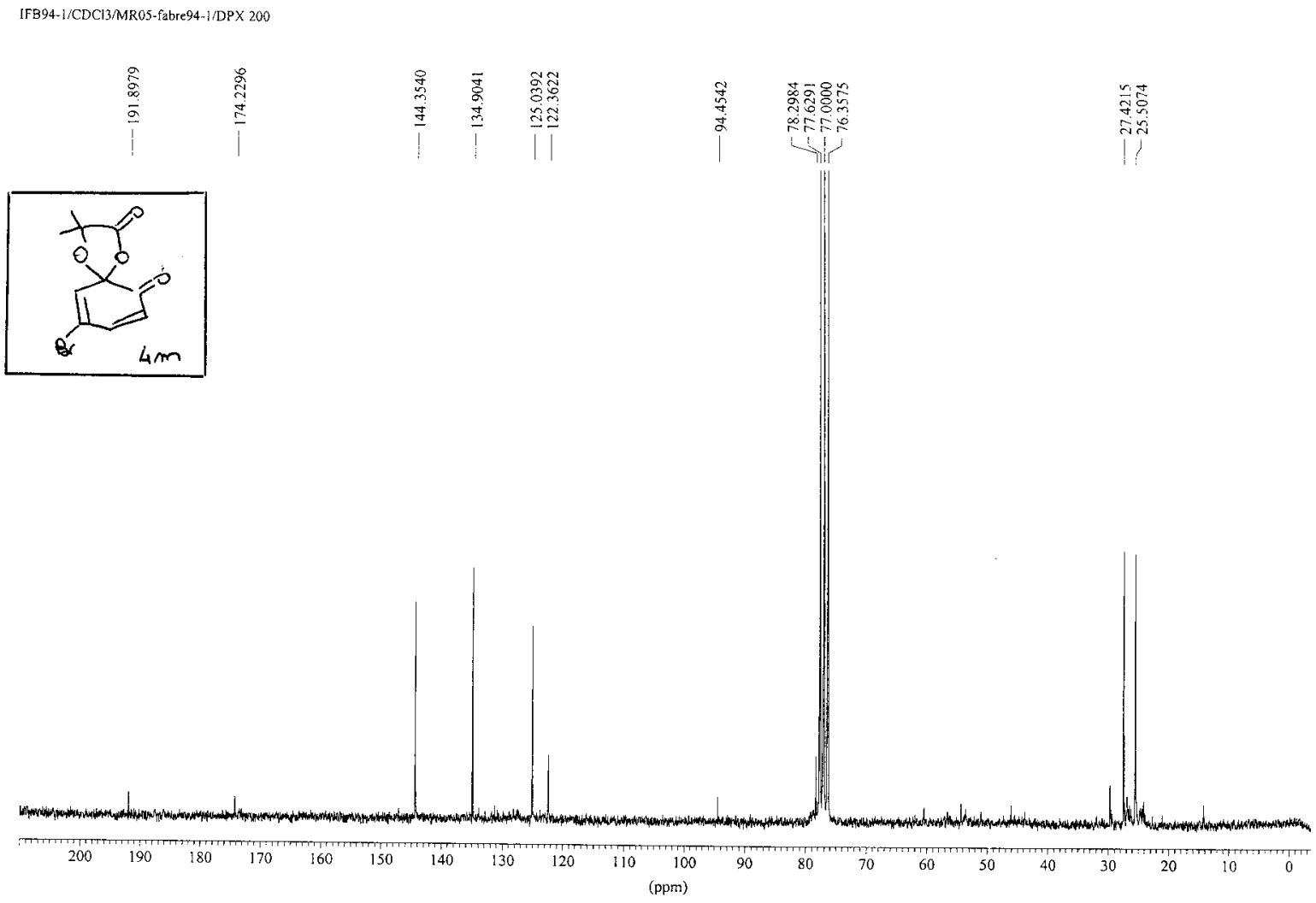

DDjoKCDCl3/19070415idpx 34ks

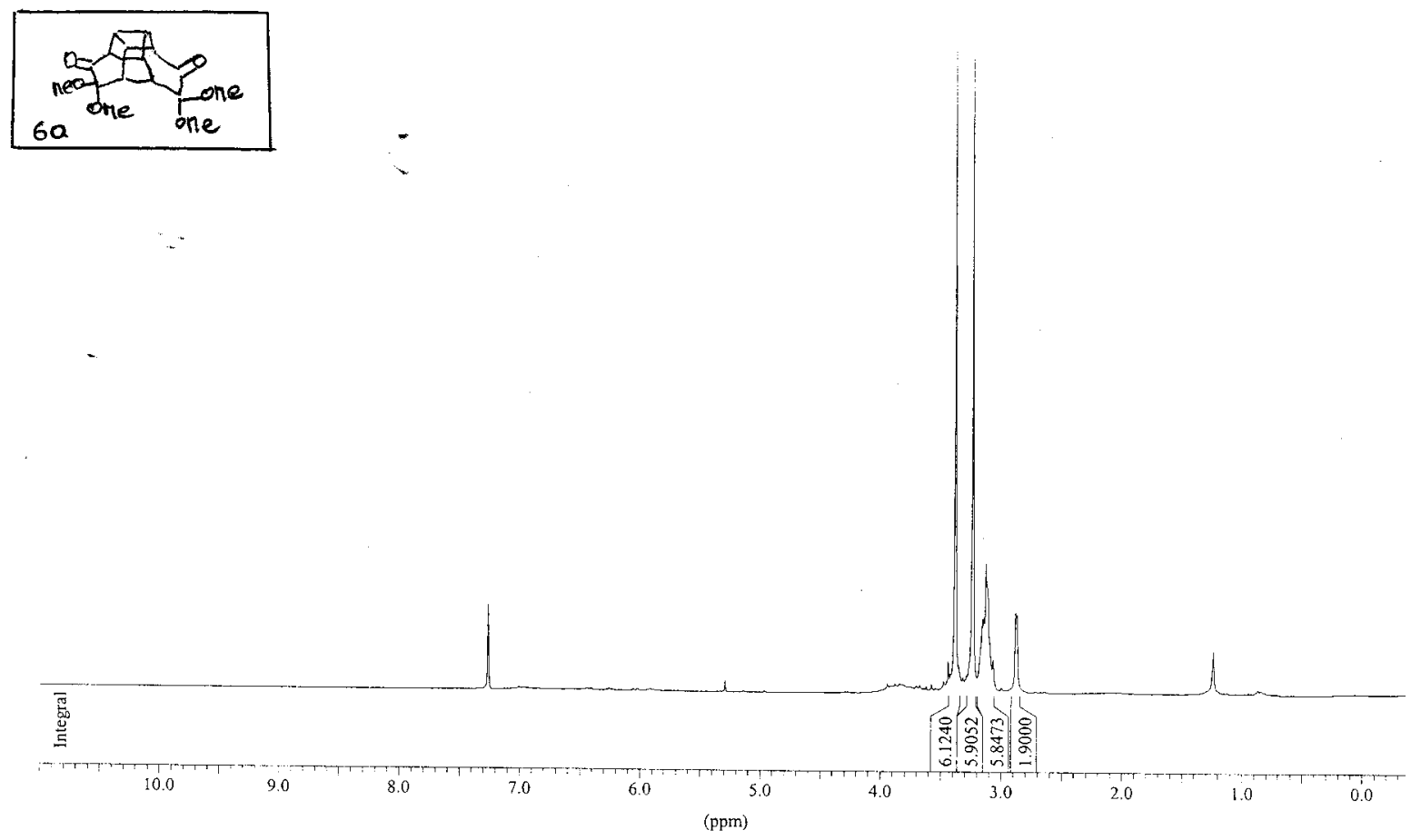

S74 
File Name

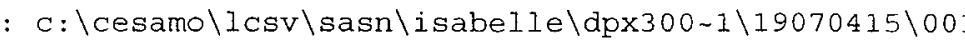

Peak Results saved in File: Nucleus

$\mathrm{SF}$

OFFSET

SW_p

$S I$ off

: $300.130007 \mathrm{MHz}$

: $19.7618 \mathrm{ppm}$

: $6172.84 \mathrm{~Hz}$

Peak Picking parameter

Peak constant $\mathrm{PC}=1.00$

Noise = 263

Sens. level $=1051$

Peak Picking region

\begin{tabular}{rrrrr}
\multicolumn{1}{c}{ Start $(\mathrm{ppm} / \mathrm{Hz})$} & \multicolumn{2}{c}{ End $(\mathrm{ppm} / \mathrm{Hz})$} & MI $(\%)$ & MAXI (\%) \\
\hline $2.99 / 898.3$ & $2.70 / 811.7$ & 5.73 & 26.10 \\
$3.62 / 1086.3$ & $2.93 / 878.4$ & 79.78 & 143.01 \\
$2.91 / 873.8$ & $2.86 / 885.5$ & 8.46 & 21.65 \\
$3.22 / 867.7$ & $2.98 / 894.2$ & 5.09 & 34.37 \\
\hline
\end{tabular}

Peak Picking results

\begin{tabular}{rrrrrr} 
Peak Nr. Data Point & Frequency & PPM & Intensity & \% Int. \\
\hline 1 & 13050 & 1014.38 & 3.3798 & 362777 & 100.0 \\
2 & 13164 & 971.43 & 3.2367 & 352143 & 97.1 \\
3 & 13229 & 946.94 & 3.1551 & 41633 & 11.5 \\
4 & 13238 & 943.55 & 3.1438 & 42449 & 11.7 \\
5 & 13253 & 937.90 & 3.1250 & 72226 & 19.9 \\
6 & 13300 & 920.19 & 3.0660 & 19133 & 5.3 \\
7 & 13447 & 864.80 & 2.8814 & 45444 & 12.5 \\
8 & 13457 & 861.04 & 2.8689 & 43919 & 12.1 \\
\hline
\end{tabular}



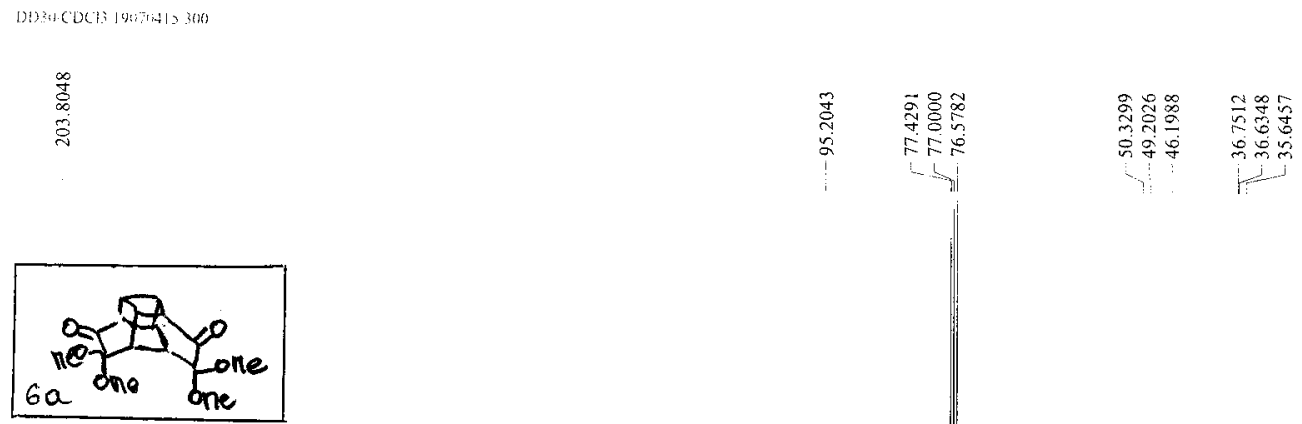

DD31/CDC 13:JL27-106AC 250
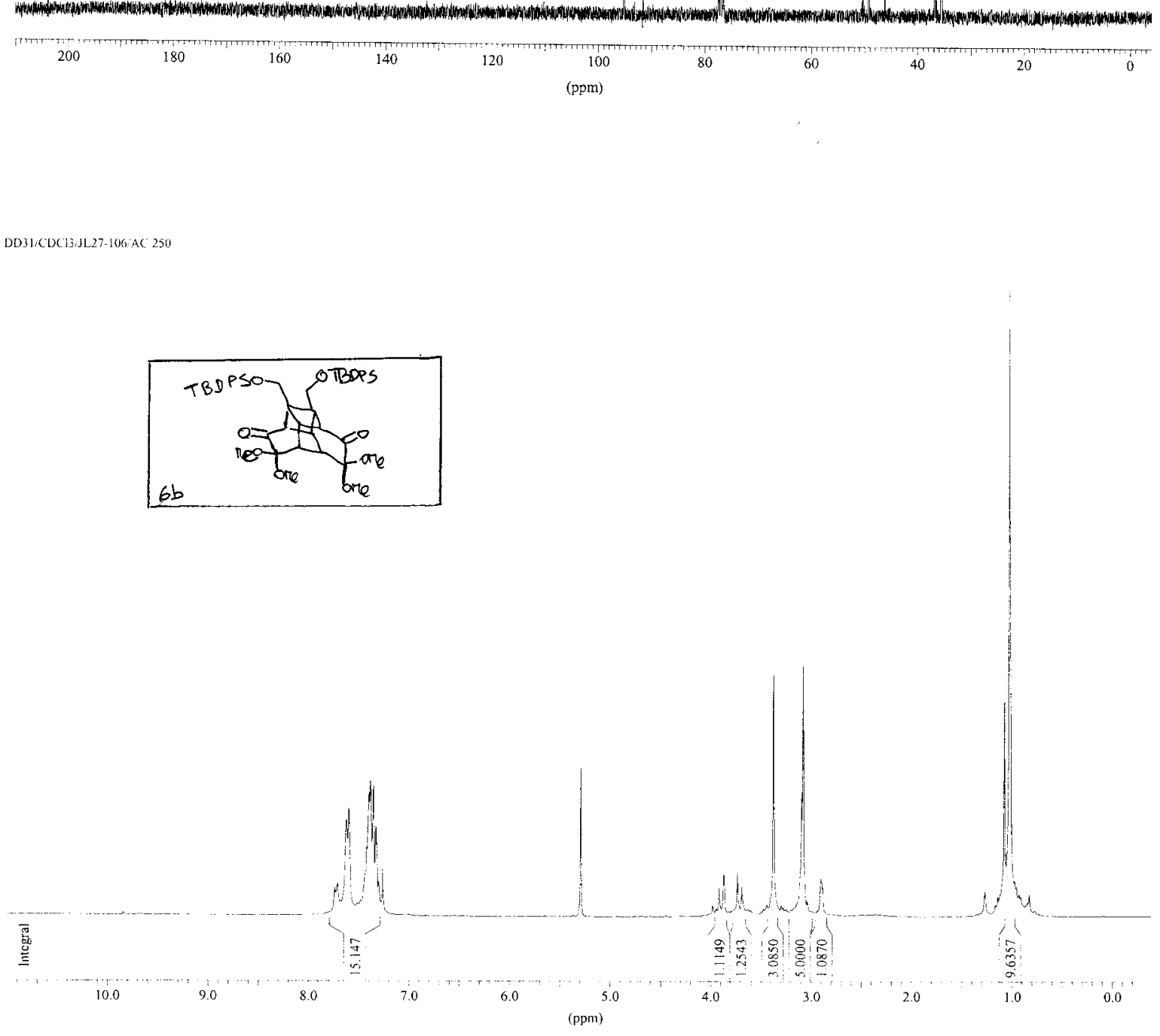


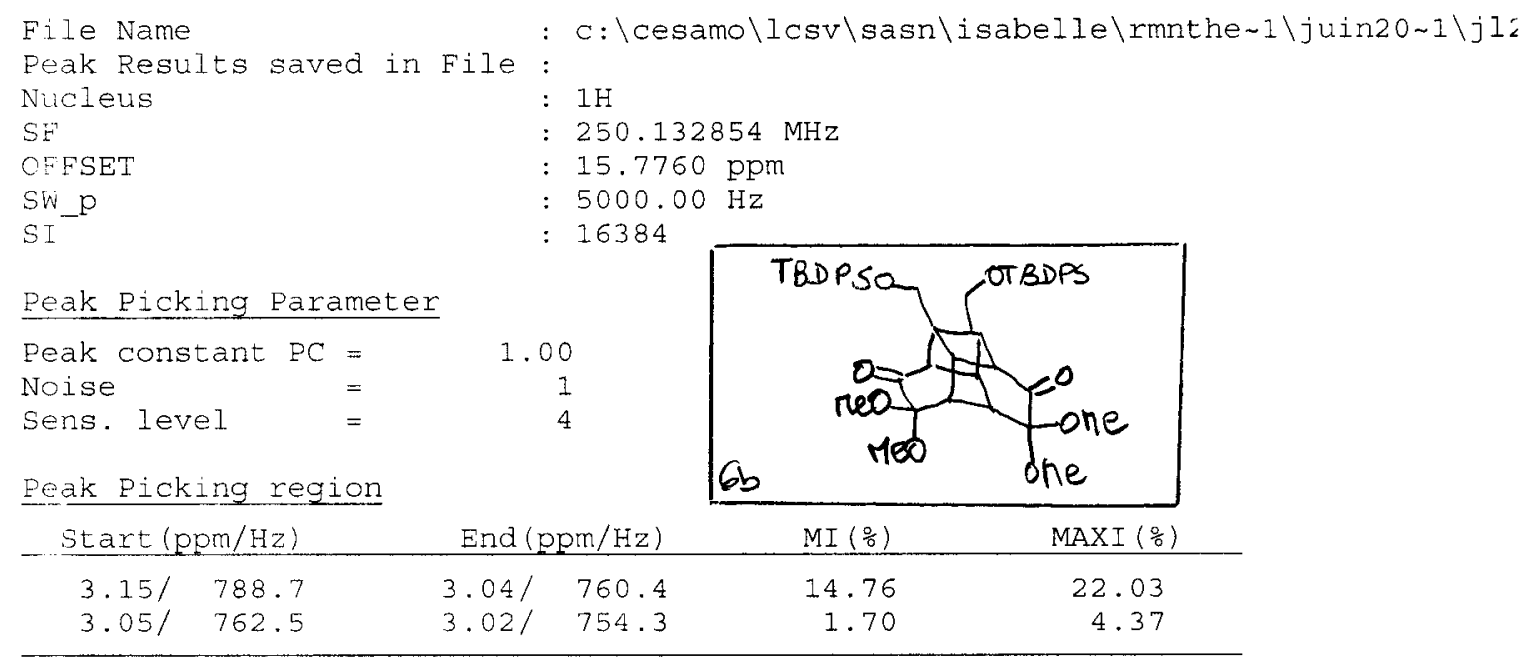

Peak Picking results

\begin{tabular}{rrrrrr} 
Peak Nr. Data Point & Frequency & \multicolumn{1}{c}{ PPM } & Intensity & \%Int. \\
\hline 1 & 6590 & 1934.98 & 7.7358 & 119 & 4.4 \\
2 & 6607 & 1929.79 & 7.7151 & 125 & 4.7 \\
3 & 6615 & 1927.35 & 7.7053 & 138 & 5.1 \\
4 & 6682 & 1906.91 & 7.6236 & 398 & 14.8 \\
5 & 6685 & 1905.99 & 7.6199 & 400 & 14.9 \\
6 & 6704 & 1900.19 & 7.5967 & 448 & 16.7 \\
7 & 6848 & 1856.25 & 7.4210 & 288 & 10.7 \\
8 & 6869 & 1849.84 & 7.3954 & 517 & 19.2 \\
9 & 6880 & 1846.48 & 7.3820 & 562 & 20.9 \\
10 & 6903 & 1839.46 & 7.3539 & 559 & 20.8 \\
11 & 6925 & 1832.75 & 7.3271 & 381 & 14.2 \\
12 & 6947 & 1826.03 & 7.3003 & 146 & 5.4 \\
13 & 6980 & 1815.96 & 7.2600 & 196 & 7.3 \\
14 & 9723 & 978.87 & 3.9134 & 120 & 4.5 \\
15 & 9759 & 967.88 & 3.8695 & 176 & 6.6 \\
16 & 9872 & 933.39 & 3.7316 & 184 & 6.9 \\
17 & 9908 & 922.41 & 3.6877 & 128 & 4.8 \\
18 & 10165 & 843.98 & 3.3741 & 1002 & 37.3 \\
19 & 10396 & 773.48 & 3.0923 & 516 & 19.2 \\
20 & 10408 & 769.82 & 3.0776 & 1038 & 38.6 \\
21 & 10443 & 759.14 & 3.0349 & 67 & 2.5 \\
22 & 10552 & 725.88 & 2.9020 & 160 & 6.0 \\
23 & 12093 & 255.60 & 1.0219 & 2688 & 100.0 \\
\hline
\end{tabular}


dd $31 / \mathrm{CDC1} 3 / 260704 / 4 / \mathrm{CP} \times 300$
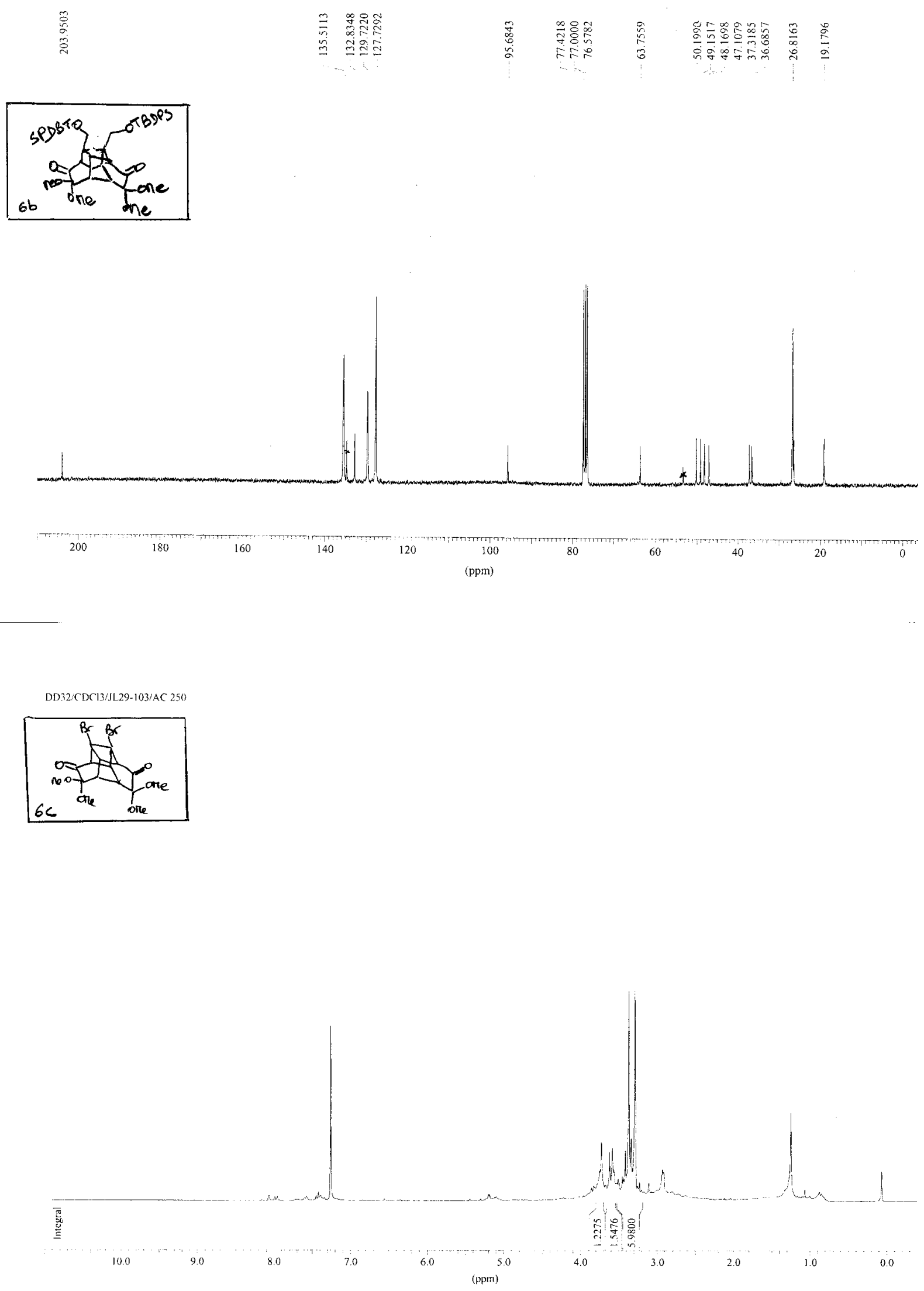

S78 
File Name

$C: \backslash W I N 1 D \backslash S P C \backslash A S P 3000 \backslash J L 29 \backslash 103001.1 R$

Peak Results saved in File:

Nucleus

$1 \mathrm{H}$

OFESET

$S W \_P$

$250.132854 \mathrm{MHz}$

$S I$

: $15.7760 \mathrm{ppm}$

$5000.00 \mathrm{~Hz}$

: 16384

Peak Picking Parameter

Feak constant PC =

1.00

Noise

$=$

3

11

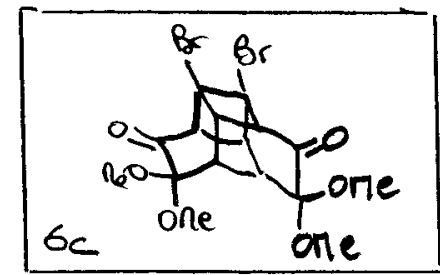

Peak Picking region

\begin{tabular}{rrrr} 
Start $(\mathrm{ppm} / \mathrm{Hz})$ & End $(\mathrm{ppm} / \mathrm{Hz})$ & MI $(\%)$ & MAXI $(\%)$ \\
\hline $9.52 / 2381.5$ & $1.84 / 460.7$ & 55.35 & 111.91 \\
$3.90 / 974.9$ & $3.04 / 759.8$ & 19.64 & 40.55 \\
$3.48 / 869.6$ & $3.17 / 792.7$ & 8.54 & 14.63 \\
\hline
\end{tabular}

Peak Picking results

\begin{tabular}{rrrrrr} 
Peak Nr. Data Point & Frequency & \multicolumn{1}{c}{ PPM } & Intensity & \% Int. \\
\hline 1 & 6980 & 1815.96 & 7.2600 & 7293 & 81.8 \\
2 & 9878 & 931.57 & 3.7243 & 2521 & 28.3 \\
3 & 9964 & 905.32 & 3.6194 & 2056 & 23.0 \\
4 & 9994 & 896.16 & 3.5828 & 2297 & 25.8 \\
5 & 10099 & 864.12 & 3.4546 & 1043 & 11.7 \\
6 & 10112 & 860.15 & 3.4388 & 1032 & 11.6 \\
7 & 10133 & 853.75 & 3.4132 & 2142 & 24.0 \\
8 & 10147 & 849.47 & 3.3961 & 1163 & 13.0 \\
9 & 10161 & 845.20 & 3.3790 & 3038 & 34.1 \\
10 & 10171 & 842.15 & 3.3668 & 8905 & 99.8 \\
11 & 10192 & 835.74 & 3.3412 & 2575 & 28.9 \\
12 & 10199 & 833.60 & 3.3326 & 2641 & 29.6 \\
13 & 10237 & 822.01 & 3.2863 & 8920 & 100.0 \\
14 & 10284 & 807.66 & 3.2289 & 825 & 9.3 \\
\hline
\end{tabular}


DD32:CDC13 JL2b-Fabre:DPX 200

\[ 1 \]
$\alpha$
$\infty$
$\infty$
$\infty$

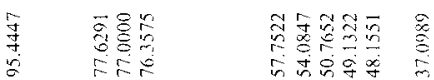

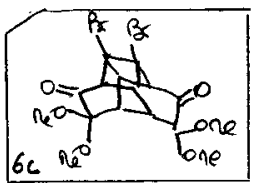

be ne Ene

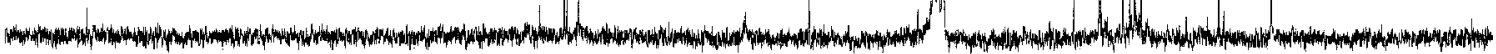

$200-180$

160

140

120

100
$(\mathrm{ppm})$

80

60

40
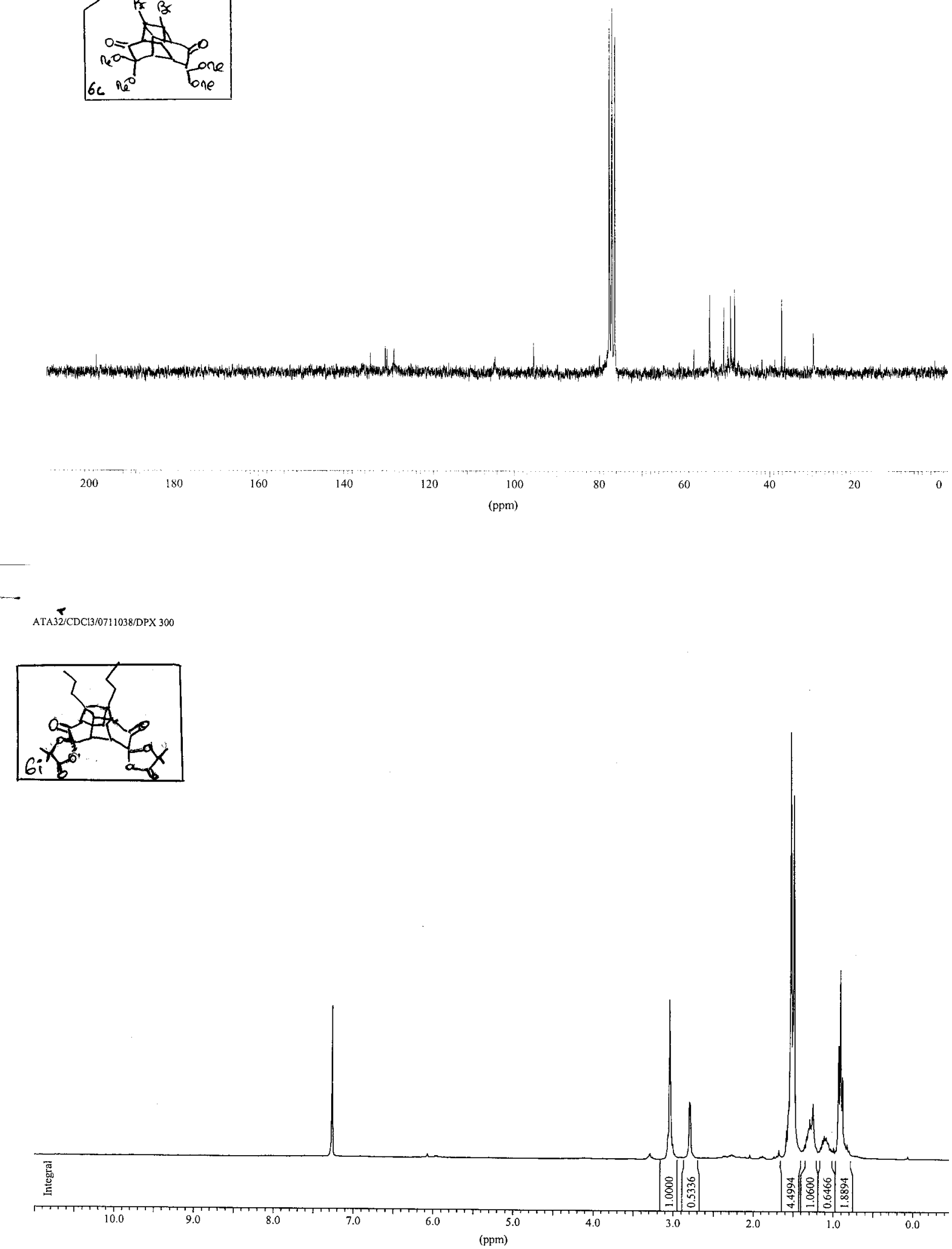
File Name

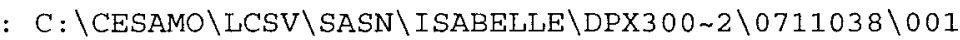
Peak Results saved in File: Nucleus

$\mathrm{SF}$ : off

OFFSET

SW_P

$S I^{-P}$

: $300.130013 \mathrm{MH}$

: $16.4170 \mathrm{ppm}$

: $6172.84 \mathrm{~Hz}$

: 32768

Peak Picking Parameter

Peak constant $\mathrm{PC}=\quad 1.00$

Noise $=226$

Sens. level = 904

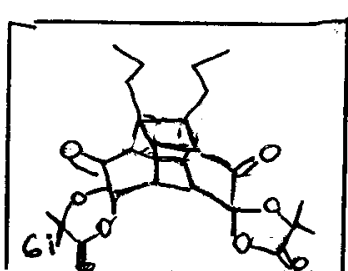

Peak Picking region

\begin{tabular}{|c|c|c|c|}
\hline Start $(\mathrm{ppm} / \mathrm{Hz})$ & End (ppm/Hz) & MI (\%) & $\operatorname{MAXI}\left(\frac{\circ}{5}\right)$ \\
\hline 2568.7 & 1869.2 & 29.79 & 43.97 \\
\hline $3.26 /$ & 683.2 & 6.76 & 43.80 \\
\hline $1.76 /$ & 362.6 & 52.18 & 100.62 \\
\hline $1.03 /$ & 217.0 & 13.54 & 55.89 \\
\hline $1.43 /$ & 336.4 & 7.71 & 20.72 \\
\hline $1.22 /$ & 306.6 & 3.85 & 7.47 \\
\hline
\end{tabular}

Peak Picking results

\begin{tabular}{rrrrrr} 
Peak Nr. Data Point & Frequency & \multicolumn{1}{c}{ PPM } & Intensity & \%Int. \\
\hline 1 & 14589 & 2178.94 & 7.2600 & 130195 & 35.2 \\
2 & 21308 & 913.22 & 3.0427 & 137690 & 37.2 \\
3 & 21702 & 839.00 & 2.7954 & 49495 & 13.4 \\
4 & 23729 & 457.15 & 1.5232 & 370107 & 100.0 \\
5 & 23786 & 446.41 & 1.4874 & 315275 & 85.2 \\
6 & 24099 & 387.45 & 1.2909 & 34837 & 9.4 \\
7 & 24119 & 383.68 & 1.2784 & 29364 & 7.9 \\
8 & 24164 & 375.20 & 1.2501 & 48377 & 13.1 \\
9 & 24355 & 339.22 & 1.1303 & 16830 & 4.5 \\
10 & 24392 & 332.25 & 1.1070 & 20600 & 5.6 \\
11 & 24429 & 325.28 & 1.0838 & 19202 & 5.2 \\
12 & 24469 & 317.75 & 1.0587 & 14811 & 4.0 \\
13 & 24678 & 278.38 & 0.9275 & 98575 & 26.6 \\
14 & 24716 & 271.22 & 0.9037 & 163898 & 44.3 \\
15 & 24754 & 264.06 & 0.8798 & 71399 & 19.3 \\
\hline
\end{tabular}


ATA32/CDC13/0711038/DPX 300

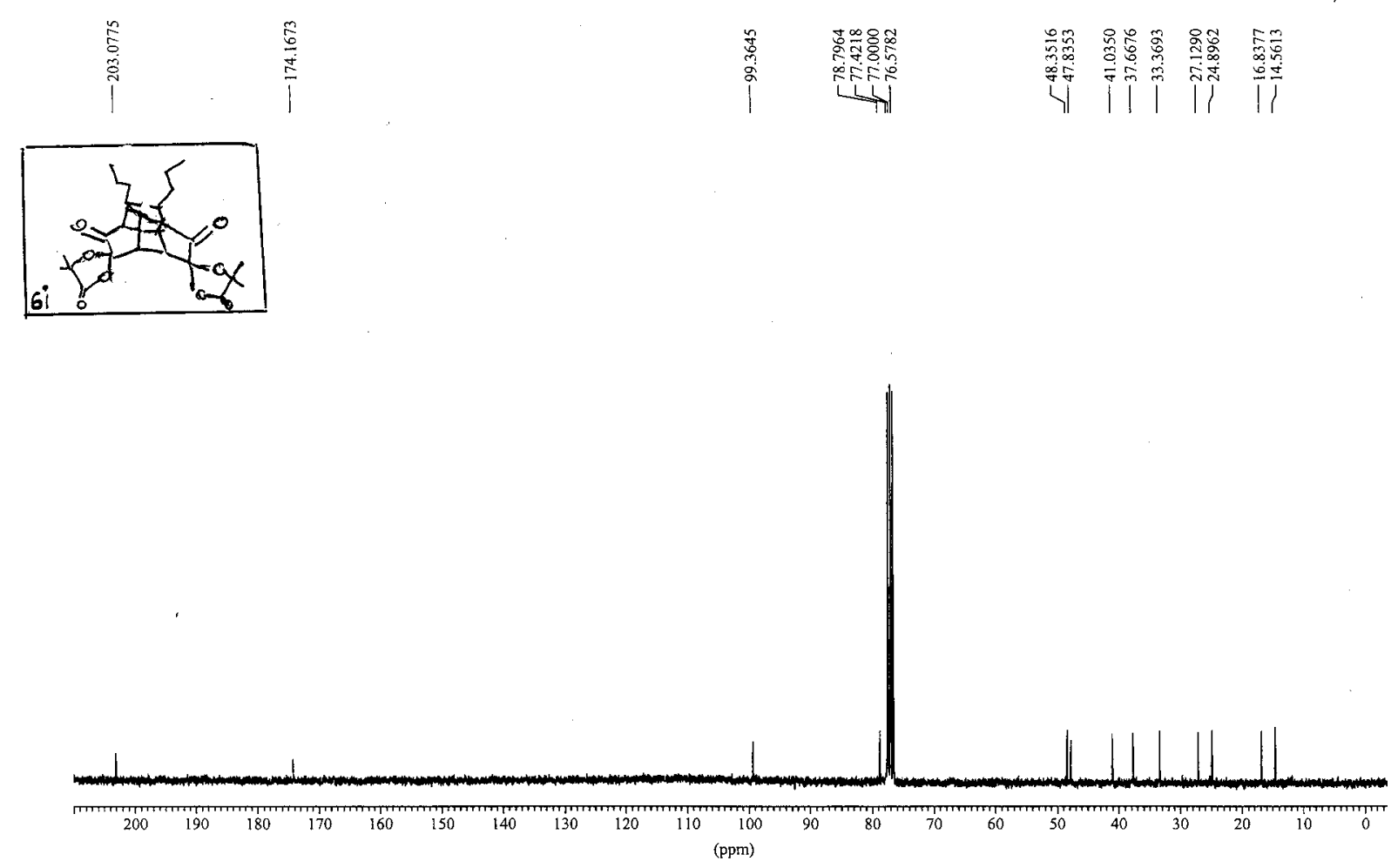

